\title{
Charge Transport in Molecular Materials: an Assessment of Computational Methods
}

\author{
Harald Oberhofer, ${ }^{*, \dagger}$ Karsten Reuter, ${ }^{\dagger}$ and Jochen Blumberger ${ }^{\ddagger}, \uparrow$ \\ †Chair for Theoretical Chemistry and Catalysis Research Center, Technische Universität \\ München, Lichtenbergstr. 4, D-85747 Garching, Germany \\ $\ddagger$ Department of Physics and Astronomy, University College London, Gower Street, London \\ WC1E 6BT, United Kingdom \\ -Institute for Advanced Study, Technische Universität München, Lichtenbergstrasse 2 a, \\ D-85748 Garching, Germany \\ E-mail: harald.oberhofer@tum.de
}

\section{Abstract}

The booming field of molecular electronics has fostered a surge of computational research on electronic properties of organic molecular solids. In particular with respect to a microscopic understanding of transport and loss mechanisms theoretical studies assume an ever increasing role. Owing to the tremendous diversity of organic molecular materials a great number of computational methods have been put forward to suit every possible charge transport regime, material and need for accuracy. With this review article we aim at providing a compendium of the available methods, their theoretical foundations, and ranges of validity. We illustrate these through applications found in the literature. The focus is on methods available for organic molecular crystals, but mention is made wherever techniques are suitable for use in other related materials such as disordered or polymeric systems.

\section{Contents}

\section{Abstract \\ 1 Introduction \\ 2 Theoretical background}

1

2
2.1 The charge carrier density . . . . 4

2.2 The charge carrier mobility . . . 5

2.3 Transport regimes . . . . . . . 6

3 Hopping Regime $\quad 7$

3.1 Polaronic rate theory . . . . . . 8

3.2 Adiabatic and non-adiabatic limit 10

3.3 Driving force, $\Delta G^{0}$. . . . . . 12

3.3.1 On-site energies . . . . . 13

3.4 Reorganization free energy, $\lambda$. . 13

3.5 Nuclear tunneling factor, $\Gamma_{n}$. . . 15

3.6 Effective frequency, $\nu_{\text {eff }} \ldots \ldots$. . 16

3.7 Electronic coupling, $H_{a b}$. . . . 16

3.7.1 The generalized MullikenHush method . . . . . . 17

3.7.2 Constrained density functional theory . . . . 18

3.7.3 Fragment orbital methods 20

3.7.4 Frozen density embedding 22

3.7.5 Block diagonalization of the electronic Hamiltonian 23

3.7.6 Analytic overlap method . 24

3.7.7 Super-exchange effective couplings ...... . 25

3.8 Kinetic Monte Carlo simulation

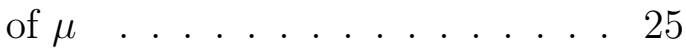

3.9 Applications . . . . . . . . . 27

4 Band transport regime 27 4.1 Effective Mass . . . . . . . . . . . . 29 


\subsubsection{Tight-binding model of} electronic band structures

4.2 Relaxation Time ........ . 31

4.3 Applications . . . . . . . . . 32

5 Intermediate regime

33

5.1 Ranges of validity . . . . . . . . 33

5.1.1 Polaronic hopping model . 33

5.1.2 Band theory model . . . . 34

5.2 Polaronic band theories . . . . . 36

5.3 Propagation of the chargecarrier wavefunction . . . . 37

5.3.1 Mean field propagation . . 38

5.3.2 Surface hopping approaches . . . . . . . 39

5.3.3 Other propagation methods 41

5.3.4 Example: propagation in a charge localized basis . . 42

5.4 The Kubo formula . . . . . . . . 44

5.5 Applications . . . . . . . . 44

\section{Synopsis, remaining challenges and} future directions

\section{References}

\section{Introduction}

Organic molecular solids have attracted much interest in the last two decades in diverse disciplines ranging from the medical sciences to semiconductor industry. Due to the excessive range of possible molecular constituents, molecular solids can show an exceptional variety of properties. In pharmacology, for instance, molecular solids have for a long time been of great importance due to the fact that most medical drugs are delivered in the form of molecular crystal powders. Thus, structure, isomorphism and solubility of the molecular drug components are the main points of interest and under active research both experimentally and theoretically. Much more recently though, organic solids have been discovered for use as electronic components ushering in the advent of the field of molecular electronics. With organic molecules as their main component, molecular electronics holds great promise with regards to a favorable economic footprint and novel materials properties. ${ }^{1-6}$ At present, applications of organic solids are mainly divided into three different branches of electronics. First, classical electronics in the form of organic field effect transistors (OFETs), which for some applications can replace standard inorganic components based on silicon. The second big field of interest is the use of molecular electronics in light harvesting and power conversion applications in the form of organic photovoltaics (OPVs). And last, not least the inverse process, i.e. the emission of light under the action of an electric current. This has been successfully deployed to the markets in the form of organic light emitting diodes (OLEDs).

Supplementing experiments, computational theory can play a major role in studying, understanding and exploring new organic molecular compounds in all these application fields. Theoretical approaches can thereby broadly be classified as follows:

1. Material optimization: The objective is to determine in detail the mechanisms at play in order to identify efficiencylimiting loss processes. Based on a thorough understanding of one material one can try to eliminate losses through modifications of the material at hand.

2. Materials screening: Given the availability of a good descriptor, which correlates well with the desired property of the material, the objective is to screen different materials for their suitability in a certain application. Considering that the space of possible molecular compounds is many orders of magnitude larger than the range of organic solids hitherto characterized this often requires an additional crystal structure prediction step - which remains a major challenge to this day.

Both kinds of approaches rely to varying degrees on a sufficiently accurate theoretical description of the underlying atomic-scale processes involved in the desired property. Yet, simultaneously they have to be able to yield experimentally accessible observables to be of practical use. 
An important figure of merit in all three electronic applications of organic solids-OFETs, OPVs, and OLEDs - is the electric conductivity $\sigma$. Simplistically, it is defined in terms of Ohm's classic law

$$
I=\sigma V,
$$

where $I$ is the current and $V$ the applied voltage. Any predictive-quality theoretical approach needs to determine $\sigma$ (or indeed at least any of its constituent parameters as outlined below) as a function of the microscopic structure and dynamics of the molecular solid. In doing so one is generally confronted with severe challenges. Among these, notably the nature of charge carriers and their transport mechanism still remains unclear in many molecular materials. Furthermore, a correct description of structure and thermal fluctuations of the molecules requires accurate molecular models, while the calculation of material properties often necessitates system sizes beyond the reach of modern computational capabilities.

While earlier reviews detailed in great length specific aspects of simulation methodologye.g. for specific systems, ${ }^{7-14}$ in this work we aim to give a comprehensive overview of theoretical methods that are presently established to tackle these problems and thus enable the computation of charge conduction in molecular solid semiconductors. Different methods have been established, depending on the prevailing transport mechanism within the material. One generally distinguishes the three regimes of charge hopping, band transport and intermediate cases. For each of these regimes we outline the methods put forward to compute the respective parameters from first principles, as well as tight-binding and semi-empirical methods. We review their theoretical underpinnings and point out similarities and differences. We illustrate, where possible, each approach with instructive examples and give an overview over applications of the respective methods found in literature. Hereby, we mainly focus on ordered organic solids, but will point out below, which of the methods discussed are also applicable to disordered systems like organic polymer blends.
Finally, one has to appreciate the importance of accurate structural models as a basis for all of the following atomic-scale simulation techniques. There can be no atomistic simulation of charge-carrier transport without a detailed knowledge of the position of all atoms involved. In the context of ordered organic crystals, especially considering material screening studies, this leads to two distinct approaches. On one hand, structural models can be taken from experimental measurements, collated for example in the Cambridge Structural Database (CSD), ${ }^{15}$ and used in computational screening studies. ${ }^{16}$ On the other hand, for systems that have either not yet been synthesized or structurally resolved through experiment, one can attempt to gain structural models from purely theoretical considerations. Such structure prediction approaches, based on either specially parametrized force fields or electronic structure methods, have shown tremendous progress over the recent years as evidenced e.g. by the crystal structure prediction (CSP) blind test series of publications. ${ }^{17-22}$ While all CSP methods are based on global geometry optimization approaches, ${ }^{23,24}$ different approaches to resolve the material structure have to be utilized for disordered systems, such as e.g. polymer blends. There, structures are generally taken as snapshots from high-temperature molecular dynamics (MD) simulations, at present predominantly performed on the level of classical force fields. ${ }^{25-28}$ In the following we will concentrate on methodological approaches for the simulation of the charge carrier dynamics given specific geometries as is appropriate for crystalline molecular solids. A generalization to the application to individual geometry snapshots is conceptually straightforward.

In compiling a review such as this one, one quickly realizes that the number of ways in which the problem of conductivity in organic molecular crystals has been approached is quite overwhelming. While it is to some extent possible to show that the multiple definitions of say a basic quantity like the mobility are all equivalent, the computational as well as experimental methods to determine it are diverse, numerous and partly beyond comparison. ${ }^{10}$ There is def- 
initely no single "best" way to approach this problem. Instead there is a rather complete (and partially even redundant) "toolbox" of methods one has to choose from for each given problem at hand. With the present manuscript we aim to provide a helpful overview over this toolbox, which aids the reader with choosing the theoretical approach best suited to their respective needs.

\section{Theoretical background}

Regardless of the underlying mechanism of charge transport, the conductivity of any material $\underline{\boldsymbol{\sigma}}$ - which in general is a tensor of rank two - is generally determined by the density $\rho_{\mathrm{c}}$ of mobile charge carriers and their mobility $\underline{\boldsymbol{\mu}}$

$$
\underline{\boldsymbol{\sigma}}=q \rho_{\mathrm{c}} \underline{\boldsymbol{\mu}},
$$

where $q$ denotes the charge of the carrier. The detailed transport mechanism enters then through the different dependence of $\rho_{\mathrm{c}}$ and $\underline{\boldsymbol{\mu}}$ on elementary parameters such as temperature or doping. A thorough computational assessment of charge transport properties of a molecular material thus has to include an estimate of both parameters.

Concerning notation: throughout this review we use bold letters $\mathbf{r}$ to denote vectors and bold underlined letters $\underline{\boldsymbol{\sigma}}$ represent second rank $(3 \times 3)$ tensors, where columns and lines enumerate the three spatial coordinates. Letters in blackboard bold $H$, finally, denote second rank tensors of higher dimensionality, i.e. basis set expanded matrices of operators.

\subsection{The charge carrier density}

In the absence of optical excitations, the density of mobile charge carriers in a solid can essentially be separated into two contributions, $\rho_{\mathrm{c}}=\rho_{\text {in }}+\rho_{\text {ex }}$. First, the so-called intrinsic carrier density $\rho_{\text {in }}$ originating from the thermally excited states of the band structure of the ideal material; and second, a density of mobile carriers $\rho_{\mathrm{ex}}$ due to extrinsic defects in the periodic structure of the material. The latter include all kinds of non-periodicities, ranging from point defects such as vacant lattice sites (due to a missing molecule) or interstitial molecules (e.g. solvent molecules left over from the crystallization process), to larger higher-dimensional defects such as interfaces with another material. ${ }^{29}$ Additionally, doping with atoms ${ }^{30,31}$ or molecules ${ }^{32,33}$ with different electronic energy level alignment contributes to the extrinsic density of charge carriers.

From a computational point of view the density of intrinsically mobile charge carriers can simply be determined from the ideal system's Fermi level, $\varepsilon_{\mathrm{F}}$, and electronic density of states (DOS), $\rho(\varepsilon)$. The latter is a measure of the states existing in the system at a given energy $\varepsilon$, while the former is defined as the chemical potential of electrons in the solid. The densities of mobile electrons $\rho_{\text {in }}^{\mathrm{e}}$ in the conduction band and electron-holes $\rho_{\mathrm{in}}^{\mathrm{h}}$ in the valence band are given by ${ }^{34}$

$$
\begin{aligned}
& \rho_{\mathrm{in}}^{\mathrm{e}}=\int_{\varepsilon_{\mathrm{F}}}^{\infty} d \varepsilon \rho(\varepsilon) f(\varepsilon ; T) \\
& \rho_{\mathrm{in}}^{\mathrm{h}}=\int_{-\infty}^{\varepsilon_{\mathrm{F}}} d \varepsilon \rho(\varepsilon) f(\varepsilon ; T),
\end{aligned}
$$

where

$$
f(\varepsilon ; T)=\frac{1}{e^{-\left(\varepsilon-\varepsilon_{\mathrm{F}}\right) / k_{\mathrm{B}} T}+1}
$$

is the Fermi-Dirac distribution function for fermionic (quasi-)particles such as electrons and holes. Here, $T$ denotes the temperature and $k_{\mathrm{B}}$ is Boltzmann's constant. A sketch outlining the thermal effects on the availability of mobile charge carriers is given in Fig. 1.

Extrinsic contributions to the carrier density, on the other hand, are not necessarily as straightforward to include in a computational model. Without explicitly accounting for the dopant or defect electronic levels, a minimalistic description is simply through a change of the Fermi-level position. Depending on the sign of the change this then results in a higher electron or electron-hole carrier density as described by eqs. (3) and schematically illustrated in Fig. 1. 


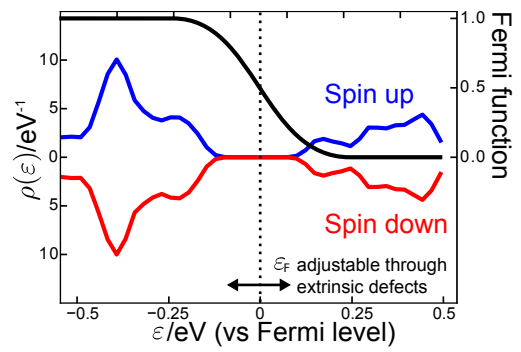

Figure 1: Sketch of the effect of finite temperatures on the distribution of occupied electronic states. Shown are representative densities of states for both spin-up (blue line) and spindown electrons (red line) for an artificial system with a bandgap of $\approx 0.2 \mathrm{eV}$. At non-zero temperatures thermal excitation described by the Fermi distribution (black line) can lead to the appearance of occupied states above and unoccupied states below the Fermi level.

\subsection{The charge carrier mobility}

The charge mobility $\boldsymbol{\mu}$ is defined as the velocity response of a charge carrier to an external electric field ${ }^{35,36}$

$$
\mu_{i j}=\frac{\langle v\rangle_{i}}{E_{j}}
$$

where $\langle v\rangle_{i}$ denotes the $i$ th component of the time-averaged velocity $\langle\mathbf{v}\rangle$ of the carrier and $E_{j}$ is a component of the electric field vector E. In condensed systems, both $\langle\mathbf{v}\rangle$ and $\mathbf{E}$ need generally to be explicitly described as threedimensional vectors, which thus yields a $(3 \times 3)$ tensorial mobility $\boldsymbol{\mu}$. Only in isotropic media, such as liquids or fully disordered solids, the velocity response can be isotropic as well. If additionally the carrier velocity response in any direction $i$ is independent of the applied field in all directions but $i$, the mobility can be reduced to a simple scalar quantity $\mu=\frac{\langle v\rangle}{E}$, involving the magnitudes $\langle v\rangle=\|\langle\mathbf{v}\rangle\|$ and $E=\|\mathbf{E}\|$.

Note that the average velocity is not generally a linear function of the field. This means in turn that the mobility as defined by eq. (4) is typically a function of the applied electric field. As discussed in more detail in Sections $3-5$, the mobility can in fact vary strongly with E. Given inaccuracies in both experimental and theoretical studies of the mobility, this can fur- ther complicate a direct comparison. For such a comparison it is therefore often useful to linearize $\langle\mathbf{v}\rangle$ with respect to the field ${ }^{37}$

$$
\mu_{i j}=\frac{\langle v\rangle_{i}}{E_{j}} \approx \frac{1}{E_{j}} \frac{\partial\langle v\rangle_{i}}{\partial E_{j}} E_{j}=\frac{\partial\langle v\rangle_{i}}{\partial E_{j}} .
$$

Note that both definitions of $\boldsymbol{\mu}$, eqs. (4) and (5), remain finite even for vanishing field, since as $E \rightarrow 0$ so does the average drift velocity. Yet, for numerical reasons it can be advantageous to employ eq. (5) in low-field regimes (vide infra). ${ }^{38}$

Experimentally, the charge carrier mobility is accessed by obtaining the response of the carrier drift velocity to an external electric field. Arguably the most direct method to measure bulk mobility is thereby the time-of-flight (TOF) technique $^{39-43}$ introduced already in the late 1960s. ${ }^{44}$ In this method the transit time $\tau_{\text {tr }}$ of a charge carrier through a solid is measured by analyzing the transient behavior of the current as the electric field is switched on. For the onedimensional case, the mobility is then simply given as $\mu=L /\left(\tau_{\operatorname{tr}} E\right)$, where $L$ is the extension of the solid. ${ }^{45}$

There are, of course many more experimental approaches available to measure carrier mobilities, each differing in experimental setup as well as accessible charge transport regime. ${ }^{46}$ Next to TOF measurements other, partly even more prominent examples are the measurement of the Hall effect mobility, ${ }^{47,48}$ the analysis of field effect transistors, ${ }^{49}$ the family of space charge limited current techniques, ${ }^{50}$ time-resolved microwave conductivity measurements, ${ }^{51}$ and finally the method of charge extraction with a linearly increasing voltage. ${ }^{52,53} \mathrm{An}$ in-depth review of these techniques is beyond the scope of this review but exhaustive discussions can be found e.g. in reviews by Tiwari and Greenham, ${ }^{46}$ Coropceanu et al.,${ }^{10}$ or a paper by Shuttle and co-workers. ${ }^{49}$

Finally, an important connection between the general concept of a charge carrier mobility of eq. (4) and classical kinetic theory is given by the Einstein-Smoluchowski (ES) equation

$$
\mu_{i j}^{\mathrm{ES}}=\frac{D_{i j}(\mathbf{E}) q}{k_{\mathrm{B}} T}
$$


which relates the mobility of a charged particle to its - generally field dependent and anisotropic-diffusion coefficient $\underline{\mathrm{D}}$ and the thermal energy $k_{\mathrm{B}} T$. In principle, this is an exact classical connection based on a statistical treatment of the carrier particle's random walk through the medium. ${ }^{54}$ Possible approximations occur in estimating $\underline{\mathbf{D}}$, e.g. based on the underlying charge transport mechanism (see below). The definition of the elements $D_{i j}$ of the diffusion tensor in anisotropic media follows from a generalization of Fick's law ${ }^{55,56}$

$$
D_{i j}=\int_{t=0}^{\infty} d t\left\langle v_{i}(t) v_{j}(0)\right\rangle
$$

where $v_{i}(t)$ is the $i$-th component of the particle velocity vector $\mathbf{v}$ at time $t$. Equation (7) is the anisotropic generalization of the well known Green-Kubo equation for isotropic media ${ }^{54}$

$$
D=\frac{1}{n} \int_{t=0}^{\infty} d t\langle\mathbf{v}(t) \cdot \mathbf{v}(0)\rangle \quad
$$

where $n$ is the spatial dimensionality. In the long time limit it reduces to the familiar definition of the diffusion constant as the time derivative of the mean square displacement (MSD) of the charge carrier ${ }^{57}$

$$
D=\frac{1}{2 n} \lim _{t \rightarrow \infty} \frac{d \operatorname{MSD}(t)}{d t} .
$$

Note that this isotropic case is given here only for comparison. It rarely applies to molecular crystals due to the different relative orientations of the molecules along the crystallographic axes. Yet, instead of the full $(3 \times 3)$ diffusion matrix it is often instructive to transform $\underline{\mathrm{D}}$ into the diagonal principal axis representation. This is always possible because the diffusion tensor is by definition positive definite and symmetric. In the low-field regime these diagonal components of the diffusion tensor can be expressed as the product of mean free path $\mathbf{l}$ and the thermal velocity of the carrier particle $v_{i}^{\text {th }},{ }^{37}$

$$
D_{i i}=l_{i} v_{i}^{\text {th }}
$$

To illustrate the connection between the Einstein mobility and eqs. (4) and (5) we depict in

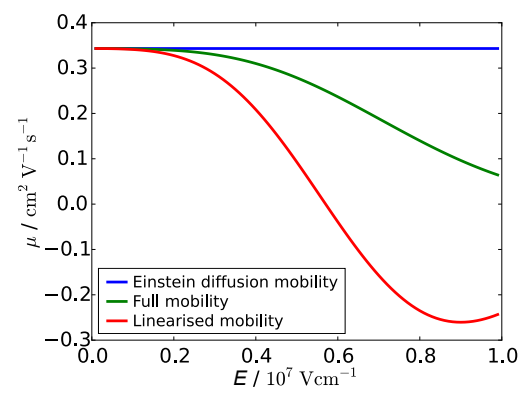

Figure 2: Comparison of definitions of the charge carrier mobility for a one-dimensional Marcus hopping model ( $v^{\text {th }}=l k$ with $k \propto$ $\left\|H_{a b}\right\|^{2} \times \exp \left(-(\lambda-q E d)^{2} / \lambda k_{\mathrm{B}} T\right)$, cf. Section 3 for a full definition) for parameters $H_{\mathrm{ab}}=$ $50 \mathrm{meV}$ and $\lambda=150 \mathrm{meV}$, representative for many organic solids. The Einstein mobility plot is based on eqs. (6) and (10), where the latter holds in the case of vanishing fields. Compared to the full mobility expression (eq. 4) and the linearized expression (eq. 5), this is clearly not valid for higher field strengths.

Fig. 2 the resulting mobilities as a function of the external field for a simple one-dimensional hopping model $\left(v^{\text {th }}=l k\right.$, with $k$ the hopping rate, vide infra). Note that the equality of all three definitions for $E \rightarrow 0$ is here indeed not a coincidence, but rather an exact property of the employed hopping model. In general, such a perfect coincidence is not guaranteed though for all regimes of condensed-phase charge transport. As such the choice of mobility definition has to be treated with care and possibly be adapted to specific experimental references. Thereby the full or linearized definitions are most often used in the context of the TOF technique ${ }^{41,42}$ and the Einstein expression most strongly associated with, e.g., field effect transistor measurements. ${ }^{46,49}$ Of the two fielddependent definitions eqs. (4) and (5) one can expect the linearized version to perform better in numerical simulations at very low fields due to the $E^{-1}$ term appearing in the general mobility definition.

\subsection{Transport regimes}

While the definition of charge carrier mobility given in eq. (4) is general, the computa- 
tional approaches to determine it vary depending on the transport mechanism prevailing in the material under consideration. In terms of this mechanism three regimes are typically discussed, which differ in the degree of the carrier localization. Phrased differently, there are in fact two opposite limiting regimes (localized hopping vs. itinerant band transport) and an intermediate regime featuring intermediate characteristics of both limits.

In the limit of polaronic or hopping transport, localized charge carriers move through the material by hopping from one site to the other, where the microscopic meaning of "site" will be further specified below. This regime applies if such localized charge carriers exist and if there is an activation barrier separating the localization sites. This is generally the case either in weakly coupled organic crystals or in disordered solids such as polymer melts. Within the hopping regime there are a number of analytical expressions to calculate the mobility, for instance based on rate theories. A variety of computational approaches aims at providing the parameters entering these expressions and we will survey these approaches in Section 3.

Band transport is the opposite limiting regime in terms of carrier localization. Here, carriers exist fully delocalized at the (valence or conduction) band edges. They propagate with an effective mass corresponding to the inverse of the band curvature and thus to the coupling between crystal sites. Propagation is limited through impurities and collisions with phonons. This regime generally applies for ultra-pure organic crystals with large electronic coupling between sites. In this case the mobility can also be calculated from analytical expressions with parameters accessible for instance through $a b$ initio calculations. The theory and computational approaches geared to conduct band mobility calculations will be outlined in Section 4.

Experimentally, one can distinguish between these two classic regimes by observing the mobility as a function of temperature. An experimentally determined increase of the mobility with temperature $T$ is usually assigned to activated hopping whereas a decrease of- ten points to band transport due to increased electron-phonon interactions. Unfortunately, carrier transport in organic molecular crystals does often not adhere exactly to one or the other regime, but instead may follow a mixture of both. For this intermediate regime no simple analytical approach to calculate the mobility exists to date. Rather one has to explicitly follow the dynamics of the carrier migration through the system to simulate the mobility directly. The state of the art of such approaches is discussed in detail in Section 5. To avoid any confusion the discussion in all subsequent Sections will thereby concentrate on the full definition of $\boldsymbol{\mu}$ as given in eq. (4).

\section{Hopping Regime}

Hopping transport models assume that the charge carrier is localized on a site in the solid, and moves from one site to another by a series of discrete jumps. Depending on the degree of localization, a site in an organic molecular solid can thereby be a single molecular unit, a part of a larger molecule or polymer, or a discrete collection of molecules. The localization can in general be induced by three distinct mechanisms. Small polaron theory predicts a localization of the charge carrier due to its interaction with the medium. The charge induces a local nuclear distortion of a molecular site and the surrounding medium which, in turn, stabilizes the localized charge. The quasi-particle of charge carrier and lattice distortion thus formed is called a small polaron. Recently, another theory for the occurrence of localized carriers has been put forward by Troisi and co-workers, ${ }^{58,59}$ based on the seminal work by $\mathrm{Su}$, Schrieffer and Heeger, ${ }^{60}$ as well as work by Hannewald and co-workers. ${ }^{61}$ In this theory localization is induced by thermal fluctuations of the coupling between sites (so called nonlocal electron-phonon coupling). Finally, in less ordered systems, charges can also localise simply through the static disorder of the underlying structure, ${ }^{62}$ which e.g. breaks conjugation in organic polymer systems.

Regardless of the underlying localization 
mechanism, hopping models describe the charge transfer (CT) through the discrete carrier jumps in terms of charge transfer rates $k_{a b}$ between the discrete sites $a$ and $b$. As detailed below, these rates depend on the specific relative orbital geometry of initial and final site and are thus in general highly site specific and directionally dependent. The average drift velocity can be written as a sum over all possible site-to-site hopping rates times the respective distance vectors. For the $i$ th component $\langle v\rangle_{i}$ of an ideal solid composed only of sites of one equivalent type, this yields $\langle v\rangle_{i}=\sum_{a b} k_{a b} d_{a b, i}$, where the sum enumerates all possible combinations of initial site $a$ and final site $b$, and $\mathbf{d}_{a b}$ denotes the center-of-charge distance vector between the sites. In general, the specific rates also show a dependence on the applied external field, which together with eq. (4) yields the following expression for the hopping mobility ${ }^{37,63}$

$$
\mu_{i j}=\sum_{a b} d_{a b, i} \frac{k_{a b}}{E_{j}} .
$$

Note that this expression applies only to perfectly ordered crystals where all sites are equivalent. In the presence of defects or even in the mere case of ideal crystals with multiple inequivalent sites per unit cell, the intricate interplay between different hopping rates and sites can no longer be easily described analytically. Instead, the reaction network defined by pairs of localization sites and their hopping rates needs to be solved numerically, for instance through a kinetic Monte Carlo algorithm as described in Section 3.8 below. In either case, the critical microscopic parameters required to determine the mobility are next to the crystal structure (which provides the site pairs and distance vectors) the charge transfer rates $k_{a b}$. In the following we describe various rate theory approaches that have been put forward to the calculation of these charge transfer rates, as well as to the calculation of the quantities that arise within the corresponding rate theory expressions.

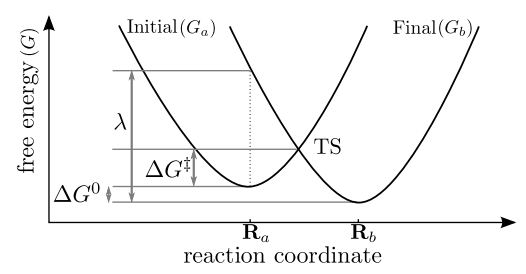

Figure 3: Schematic of a two-state polaronic charge transfer model, where the reaction coordinate is a collective variable of all nuclear coordinates $\mathbf{R}$. Initial and final electronic states are described by free energy curves $G_{a}(\mathbf{R})$ and $G_{b}(\mathbf{R})$, respectively. Both curves meet at the transition state marked TS. The curves have minima at the reaction coordinates $\mathbf{R}_{a}$ and $\mathbf{R}_{b}$ which are, in general, energetically separated by $\Delta G^{0}=G_{b}\left(\mathbf{R}_{b}\right)-G_{a}\left(\mathbf{R}_{a}\right)$. For further details see text.

\subsection{Polaronic rate theory}

Polaronic rate theories have been applied to a multitude of condensed matter systems. The main argument underlying these theories is that in order to obey energy conservation in a closed system, the charge transfer itself (the fast mode of the system) can only occur at a transition state (TS) at which the nuclear configurations (the slow mode) of initial and final polaronic state are energetically degenerate. For a charge transfer to occur, the system must therefore overcome a barrier which physically arises from the polarization of the surrounding medium to a given charge state - at least partially, as will be discussed later.

Assuming a harmonic response one can visualize this process in the form of two parabolae as depicted in Fig. 3. Each parabola describes the variation of the free energy upon changes of the nuclear degrees of freedom along a suitable reaction coordinate, while simultaneously constraining the electronic state of the system to the one at the minimum of the parabola. Each parabola thus represents a so-called diabatic state of the system; one for the initial state with the charge at site $a$ and one for the final state with the charge at site $b$. Figure 3 then defines three important energetic quantities. First, the driving force $\Delta G^{0}$ between initial polaronic state $a$ and final polaronic state 
$b$ at their respective minima of the reaction coordinate. Second, the diabatic activation energy $\Delta G^{\ddagger}$ necessary to move the system from the initial polaronic state $a$ to the TS. Finally, the reorganization free energy $\lambda$ which describes the energy cost to bring the nuclear configuration of the polaronic state $b$ into that of state $a$ while keeping the electronic configuration fixed at state $b$. All free energies are thereby dynamical averages over all nuclear degrees of freedom except the reaction coordinate.

The actual choice of reaction coordinate is thereby far from arbitrary. It has long been known ${ }^{64}$ that a poorly chosen reaction coordinate can lead to an erroneous description of the reaction pathway or at least, in the case of a dynamical sampling of the process, a bad convergence of sampled quantities. ${ }^{65}$ In the case of charge carrier hopping, where the reaction coordinate has to be a collective variable describing relaxation of the surrounding medium to changes in a local charge state, Mones and co-workers ${ }^{66,67}$ recently quantified the importance of using a good reaction coordinate to avoid hysteresis and slow convergence. Fortunately, this problem had already been addressed in the early 1980s by the pioneering works of Zusman $^{68}$ and Warshel, ${ }^{69}$ who proposed to use a coordinate based on the energy gap $\Delta E$ between diabatic states. In a given nuclear geometry $\mathbf{R}$ it is defined as

$$
\Delta E(\mathbf{R})=E_{b}(\mathbf{R})-E_{a}(\mathbf{R})
$$

where $E_{a, b}(\mathbf{R})$ denote the total energy of initial and final diabatic state, respectively. It is therefore a measure for the preference of the charge carrier to localize in either state for any given coordinate and thus depends crucially on the configuration of the dielectric surroundings. Since its inception the energy gap coordinate has shown its feasibility numerous times in both classical $^{70-74}$ and quantum mechanical simulations $^{75-78}$ of charge carrier transport, and yields results that compare well also to experimental results. ${ }^{79,80}$

In the harmonic approximation, the semiclassical transition state theory (TST) expres- sion for the charge transfer rate reads $7,35,81,82$

$$
k_{a b, \mathrm{TST}}=\nu_{\mathrm{eff}} \kappa_{\mathrm{el}} \Gamma_{n} e^{-\beta\left(\Delta G^{\ddagger}-\Delta^{\ddagger}\right)},
$$

with $\beta=1 / k_{\mathrm{B}} T$. The activation energy is hereby given as

$$
\Delta G^{\dagger}=\Delta G^{\ddagger}-\Delta^{\ddagger}
$$

i.e. the diabatic activation energy is reduced by an adiabatic correction factor $\Delta^{\ddagger}$ that accounts for a finite electronic coupling between the initial state $a$ and final state $b$. The three contributions to the prefactor in eq. (13) are the effective vibrational frequency $\nu_{\text {eff }}$ along the reorganization reaction coordinate, the electronic transmission coefficient $\kappa_{\mathrm{el}}$ which accounts for a possibly less than perfect transmission of the charge once the TS has been reached, and the nuclear tunneling factor $\Gamma_{n}$. The latter is a correction accounting for quantum effects of the nuclear degrees of freedom which can lead to an overall lower effective free energy barrier and thus an enhanced total transition rate $\left(\Gamma_{n} \geq 1\right)$. Yet, these enhancements generally only play a role at low temperatures due to the large masses of the nuclei involved in the polaronic reorganization (cf. Section 3.6). ${ }^{81}$ In most studies, the nuclear enhancement factor is therefore set to one from the outset.

With the exception of $\Gamma_{n}$, all of the quantities entering eq. (13) can be expressed in terms of semi-classical Landau-Zener (LZ) theory. ${ }^{83-85}$ For the electronic transmission coefficient this leads to

$$
\kappa_{\mathrm{el}}=\left\{\begin{array}{lll}
\frac{2 P_{\mathrm{LZ}}}{1+P_{\mathrm{LZ}}} & \text { if } & \Delta G^{\dagger} \geq-\lambda \\
2 P_{\mathrm{LZ}}\left(1-P_{\mathrm{LZ}}\right) & \text { if } & \Delta G^{\dagger}<-\lambda
\end{array}\right.
$$

where $P_{\mathrm{LZ}}$ is the $\mathrm{LZ}$ transition probability for a single crossing of the transition region along the reaction coordinate. It is given by

$$
\begin{aligned}
& P_{\mathrm{LZ}}=1-e^{-2 \pi \gamma}, \\
& 2 \pi \gamma=\frac{\pi^{3 / 2}\left\langle\left|H_{a b}\right|^{2}\right\rangle_{\mathrm{TS}}}{h \nu_{\mathrm{eff}} \sqrt{\lambda k_{\mathrm{B}} T}} .
\end{aligned}
$$

Here, $h$ is Planck's constant and $H_{a b}=$ $\left\langle\Psi_{a}|\hat{H}| \Psi_{b}\right\rangle_{\mathrm{TS}}$ is the Hamiltonian transition ma- 
trix element (or electronic coupling) between initial and final diabatic electronic state at the TS. The angular brackets \langle\rangle$_{\mathrm{TS}}$ thereby denote an equivalent dynamical averaging over all nuclear configurations of the TS as done for the free energy expressions. This given form of the transition probability and specifically eq. (15c) is only valid in the harmonic (linear response) regime with identical polarization responses for initial and final state, i.e. identical curvature of both parabolae in Fig. 3. Equivalent expressions can be obtained for the general case of different (harmonic) polarization responses. ${ }^{81,83,85}$

Within this LZ theory, Spencer et al. ${ }^{86}$ showed the two contributions to the activation energy to be

$$
\begin{aligned}
\Delta G^{\ddagger} & =\frac{\left(\lambda+\Delta G^{0}\right)^{2}}{4 \lambda}, \\
\Delta^{\ddagger} & =\left\langle\left|H_{a b}\right|^{2}\right\rangle_{\text {TS }}^{1 / 2}-\frac{1}{\lambda}\left\langle\left|H_{a b}\right|^{2}\right\rangle_{\mathrm{a}},
\end{aligned}
$$

where for the adiabatic correction factor $\left(\Delta^{\ddagger}\right)$ they assumed a vanishing driving force $\Delta G_{0}$. The first term of eq. (16b) thereby corresponds to the difference between adiabatic and diabatic free energy surfaces, averaged at the TS. The second term is the equivalent free energy difference averaged at the initial adiabatic state $a$ of the charge transfer. Note that earlier work $^{14,37,38,87,88}$ employed a different approximation for this non-adiabatic correction factor, assuming that initial diabatic and adiabatic free energy minima coincide at the same reaction coordinate. Spencer et al. recently pointed out ${ }^{86}$ that this approximation is not necessarily always justified and indeed leads to an erroneous criterion on $H_{a b}$ and $\lambda$ for the validity of the TST approach (see Section 3.2 below).

As apparent from eqs. (11-16), the charge mobility within the hopping model is fully determined through the quantities $\nu_{\text {eff }}, \lambda, \Gamma_{n}, H_{a b}$, and $\Delta G^{0}$. In the case of sufficiently weak external fields which do not significantly distort the frontier orbitals of the molecules mediating the charge transfer, to a good approximation only $\Delta G^{0}$ depends on $\mathbf{E}$. This means that all other quantities can be estimated in a field-free approach. The most common computational tech- niques to determine all five parameters will be discussed in Sections 3.3 to 3.7 below.

\subsection{Adiabatic and non-adiabatic limit}

In order to appreciate the actual transport mechanism underlying hopping models in general it is instructive to consider the adiabatic and non-adiabatic limits of the presented TST rate expression in the semi-classical LZ formulation, cf. eqs. (13-16). Note that adiabatic and non-adiabatic here are taken to describe respective physical processes. To avoid confusion, the label diabatic is only applied to charge separated states which are not eigenfunctions of the Hamiltonian.

Physically, in the adiabatic limit the electronic wavefunction of the charge carrier changes slowly and gradually ("adiabatically") from the initial to the final charge localized states as the system crosses the activation barrier. ${ }^{35,37,38}$ This is the case for large electronic couplings, small reorganization energy and small nuclear velocity along the reaction coordinate. In the opposite, non-adiabatic limit, the electronic wavefunction changes almost instantly at the barrier top. This is the case for small couplings, large reorganization energies and large velocities along the reaction coordinate. ${ }^{86}$ In terms of the $\mathrm{LZ}$ parameters, the two limits thus correspond to different ratios of $H_{a b}$ to $\lambda$, and therewith to different adiabaticity factors $2 \pi \gamma$, cf. eq. (15c).

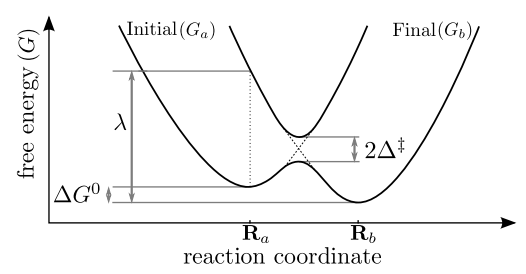

Figure 4: Schematic of the adiabatic potential energy surfaces (solid lines) for a two-state charge transfer compared to the respective diabatic picture (dashed lines).

Examining first the adiabatic limit we assume a large coupling between initial and final electronic states on the order of the reorganiza- 
tion energy (and compared to the effective frequency). This leads to an adiabaticity factor $2 \pi \gamma \gg 1$. In this case the LZ probability $P_{\mathrm{LZ}}$ approaches unity, cf. eq. (15b), which effectively means that every time the system crosses the TS region it will end up in the other charge state. Consequently, in this limit the electronic transmission coefficient $\kappa_{\text {el }}$ approaches unity, which reduces the semi-classical TST expression, eq. (13), to a standard Arrhenius form ${ }^{35,86}$

$$
k_{a b, \text { adiab }}=\nu_{\text {eff }} e^{-\beta\left(\Delta G^{\ddagger}-\Delta^{\ddagger}\right)} \Gamma_{n} .
$$

In the adiabatic limit, the prefactor thus only depends on the effective vibrational frequency along the reaction coordinate. Besides this, the only dependence of the rate on the electronic coupling is then via the adiabaticity correction $\Delta^{\ddagger}$, which effectively lowers the free energy barrier of the reaction as indicated in Fig. 4.

In the opposite, non-adiabatic limit $2 \pi \gamma \ll 1$ the exponential in the LZ probability, eq. (15b), can be expanded in a Taylor series, which, if truncated after the first order, yields $P_{\mathrm{LZ}} \approx$ $2 \pi \gamma$. In this case the probability for a charge to cross from one parabola to the other - which is proportional to the electronic coupling - is very low. In this limit, the adiabatic correction to the activation energy is much smaller than the activation energy $\left(\Delta^{\ddagger} \ll \Delta G^{\ddagger}\right)$ since in most non-adiabatic cases then, according to the definition of $\gamma$, eq. (15c), the electronic coupling is much smaller than the reorganization free energy $\left(\lambda \gg H_{a b}\right){ }^{86}$ This finally means that $\left(\lambda+\Delta G^{0}\right) \gg\left\langle\left|H_{a b}\right|^{2}\right\rangle_{\text {TS }}^{1 / 2}$ and thus the full semiclassical LZ rate equation reduces to ${ }^{35}$

$k_{a b, \text { nadiab }}=\frac{2 \pi}{\hbar} \frac{1}{\sqrt{4 \pi \lambda k_{\mathrm{B}} T}}\left\langle\left|H_{a b}\right|^{2}\right\rangle_{\mathrm{TS}} e^{-\beta \Delta G^{\ddagger}} \Gamma_{n}$.

This is the celebrated Marcus rate equation. ${ }^{89,90}$ In this limit, the rate does not depend on the effective frequency $\nu_{\text {eff }}$ anymore, but is instead fully determined through $\lambda, H_{a b}$, and $\Delta G^{0}$. In this limit the free energy curve of the process is thus indeed best described in terms of the two diabatic parabolae depicted in Fig. 3. Also note, that in this limit it is common to summarize all $\lambda$-dependent terms into the so-called Franck-Condon factor ${ }^{35}$

$$
\mathcal{F}=\frac{1}{\sqrt{4 \pi \lambda k_{\mathrm{B}} T}} e^{-\beta \Delta G^{\ddagger}} .
$$

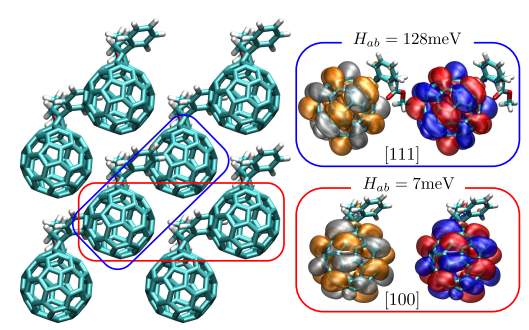

Figure 5: Left: Schematic representation of the electron acceptor Phenyl-C61-butyric Acid Methyl Ester (PCBM) in the bcc crystal structure. High and low coupling directions along [111] and [100] are indicated by blue and red boxes, respectively. Right: Donor/acceptor molecule pairs, depicting in orange/silver the donor and in red/blue the acceptor orbital along these directions, respectively. Data taken from reference. ${ }^{38}$

Even though appealing in terms of the physical interpretation of the charge transfer mechanism, it is generally dangerous to directly employ the rate expressions for the adiabatic and non-adiabatic limit, eqs. (17) and (18), respectively, in a hopping model study. This uncertainty has recently been pointed out by Gajdos et al. ${ }^{38}$ which computed LZ electronic coupling elements and reorganization free energies for the frequently used electron acceptor Phenyl-C61-butyric Acid Methyl Ester (PCBM). Depending on the crystal orientation they obtained $H_{a b}$ values between a few meV to more than one hundred meV as illustrated in Fig. 5. In contrast, $\lambda$ was shown to be more or less constant at $\sim 140 \mathrm{meV}$, which results in room temperature non-adiabaticity factors $10^{-4} \mathrm{meV} / h \nu_{\text {eff }} \leq 2 \pi \gamma \leq 2 \mathrm{meV} / h \nu_{\text {eff }}$ even within this one system.

Another important question that appears in this context concerns the validity of the hopping model itself. The initial assumption in this respect was that the excess charge (electron or hole) is localized as a small polaron on a single site. In the semi-classical LZ formulation this is the case if the activation energy for charge 
transfer, $\Delta G^{\dagger}=\Delta G^{\ddagger}-\Delta^{\ddagger}$, is positive, i.e. if a finite barrier exists at all. Conditions for the existence of a barrier $\Delta G^{\dagger}>0$ can be estimated by inserting eqs. (16) into the definition of the activation energy, eq. (14). For a vanishing driving force $\Delta G^{0}=0$ and under the assumption that the mean square coupling elements averaged in the TS and the initial state agree, $\left\langle\left|H_{a b}\right|^{2}\right\rangle=\left\langle\left|H_{a b}\right|^{2}\right\rangle_{\mathrm{TS}}=\left\langle\left|H_{a b}\right|^{2}\right\rangle_{a}$, the barrier height is then given by a simple quadratic equation $^{86}$ in $\left\langle\left|H_{a b}\right|^{2}\right\rangle^{1 / 2}$

$$
\Delta G^{\dagger}\left(\Delta G^{0}=0\right)=\frac{\left\langle\left|H_{a b}\right|^{2}\right\rangle}{\lambda}-\left\langle\left|H_{a b}\right|^{2}\right\rangle^{1 / 2}+\frac{\lambda}{4} .
$$

Hence, the activation barrier between initial and final minimum vanishes at $\left\langle\left|H_{a b}\right|^{2}\right\rangle^{1 / 2}=$ $\lambda / 2$ to yield one single delocalized state. Furthermore, while eq. (20) seems to suggest a reemergence of a barrier for values of $\left.\left\langle\left|H_{a b}\right|^{2}\right\rangle^{1 / 2}\right\rangle$ $\lambda / 2$, the corresponding minima would lie in the complex plane of the reaction coordinate, thus not describing physically relevant states. This has the effect that for such values of $\left\langle\left|H_{a b}\right|^{2}\right\rangle^{1 / 2}$ the shared minimum stays at a value of the reaction coordinate $\Delta E=0$ and gets deeper the larger the coupling. ${ }^{14,86}$ This situation is illustrated in Fig. 6 where we plot the adiabatic potential energy surface of a PCBM dimer $(\lambda=140 \mathrm{meV})$ for a range of different coupling values.

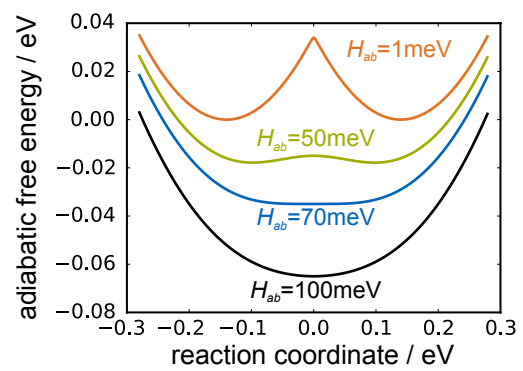

Figure 6: Adiabatic free energy profiles for a PCBM dimer $(\lambda=140 \mathrm{meV})$ for different square averaged coupling values $\left\langle\left|H_{a b}\right|^{2}\right\rangle^{1 / 2}$, here for simplicity abbreviated as $H_{a b}$. Curves were calculated with eq. (10) of reference. ${ }^{14}$ Note the disappearance of a dividing barrier between minima $a$ and $b$ for $H_{a b} \geq 70 \mathrm{meV}=\lambda / 2$.

As mentioned above, earlier studies applied different approximations ${ }^{37,38,87,88}$ to the estimation of the adiabatic correction factor thus yielding different estimates - such as the widely used condition ${ }^{14}$ of $\left\langle\left|H_{a b}\right|^{2}\right\rangle^{1 / 2}<3 \lambda / 8$ for the existence of separate initial and final states. This condition disagrees with the transition from double- to single-well potential, as illustrated in Fig. 6. Instead the approximation given in eq. (16b) should be used.

Finally, returning to the example of PCBM the $\lambda / 2$ condition yields an upper bound of $\left\langle\left|H_{a b}^{2}\right|\right\rangle^{1 / 2} \leq 70 \mathrm{meV}$ for the existence of an activation barrier and thus the validity of the hopping model. Yet, in all common PCBM crystal structures there exist nearest-neighbor pairs of molecules for which the couplings are of this order of magnitude or even higher. ${ }^{38}$ For the computational search for high mobility organic electronic materials this underscores that hopping models, although convenient and relatively cheap to parametrize from first principles, have to be applied with caution.

\subsection{Driving force, $\Delta G^{0}$}

The driving force is the difference in free energy between the fully relaxed initial and final state, i.e. the minima of the two diabatic surfaces in Figs. 3 and 4 . For a carrier of charge $q$ moving in a homogenous external field $\mathbf{E}$ between sites $a$ and $b$ along the vector $\mathbf{d}_{a b}$ it generally reads ${ }^{91}$

$$
\Delta G^{0}=G_{a}\left(\mathbf{R}_{a}\right)-G_{b}\left(\mathbf{R}_{b}\right)+q \mathbf{E} \cdot \mathbf{d}_{a b} \quad,
$$

where $G_{a}$ and $G_{b}$ are the - so-called on-sitefree energies of the state with the charge at site $a$ and $b$, respectively, while $\mathbf{R}_{a}$ and $\mathbf{R}_{b}$ are the corresponding equilibrium nuclear configurations (cf. Fig. 4). Equation (21) thereby rests on the assumption that the external electric field is weak enough-depending on molecular polarizability and orientation - not to perturb the molecular electronic structure. This means that only the third term in eq. (21) depends on the external field and the contributions $G_{a, b}$ - also called the on-site energies - can be estimated from field free simulations. They do, however, depend on the dielectric response of the entire environment of the two sites $a$ and 
$b$ involved and therefore an accurate description of both diabatic states in the extended system. In general, $G_{a, b}$ are therefore estimated directly from simulations of either state using for example a constrained electronic structure approach $^{92}$ (cf. Section 3.7.2) or sufficiently accurate classical force fields. ${ }^{93}$ A more detailed description of the calculation of site energies is given in Sec. 3.3.1 below.

In homo-molecular crystalline systems the environmental contribution is canceled by the symmetric equivalence of initial and final state though. This leaves only the contribution due to the external electric field. In this case the driving force simplifies to

$$
\Delta G^{0}=q \mathbf{E} \cdot \mathbf{d}_{a b}
$$

which can be calculated analytically for any site-to-site vector $\mathbf{d}_{a b}$.

\subsubsection{On-site energies}

As discussed above, the on-site contributions $G_{a}\left(\mathbf{R}_{a}\right)$ and $G_{b}\left(\mathbf{R}_{b}\right)$ cancel each other out in perfect homo-molecular crystals. In disordered systems or crystals with inequivalent sites, on the other hand, they have to be determined explicitly. Furthermore, as will be discussed in Sections 4.1 .1 and 5 below, other approaches to CT modelling depend on absolute values of the respective on-site contributions instead of just pairwise differences.

On-site free energies are a local property ${ }^{94}$ in that they depend on the charge being localized on a single site in the crystal, yet also include the polarization response of the surroundings. ${ }^{91}$ For a computational study, this means that to determine $G_{a, b}\left(\mathbf{R}_{a, b}\right)$, or neglecting entropic contributions, the on-site energies $E_{a, b}\left(\mathbf{R}_{a, b}\right)$, one needs to be able to construct the diabatic states $a$ and $b$ and to determine their energy in an extended system. Considering the inherent inability of e.g. DFT studies to accurately treat charge-localized states, especially in extended systems, ${ }^{95}$ on-site energies are not amenable to direct treatment by DFT. Instead a number of approximate methods have been put forward to tackle this problem. For example, Brédas et al. ${ }^{96,97}$ and Fuchs and coworkers $^{91}$ first determined the charge distributions of single molecules on the level of hybrid level DFT. This charge distribution is then inserted into a polarizeable but classical model of the periodic crystal to determine the energy of a given localized state $a$ or $b$. A similar combined quantum mechanical/molecular mechanical approach was followed by Norton and Brédas, ${ }^{98}$ yet based on the ONIOM method where the classical force field energy is corrected by quantum mechanical corrections for the central molecule. In an analogous approach, Friedrich et al. ${ }^{99}$ embedded a DFT inner region into a DFT tight-binding exterior for the determination of on-site energetic disorder. A quantum chemical approach based on a combination of the valence bond model and Hartree Fock calculations ${ }^{100}$ was also shown to not only yield accurate results for on-site energies, ${ }^{101}$ but also to reproduce their temperature variations due to crystal lattice vibrations. ${ }^{94}$ In a more approximate approach geared towards on-thefly determination of charge transfer parameters in a direct charge propagation model (see Sec. 5.3.4 below), Spencer et al. ${ }^{86,93}$ determined on-site energies purely from classical force field calculations. Finally, Nagata ${ }^{102}$ pioneered the use of a fitted charge response kernel ${ }^{103}$ to determine on-site energy disorder in amorphous Tris(8-hydroxyquinolinato)aluminium. For the same system Kwiatkowski et al. ${ }^{104}$ demonstrated a particularly simple approximation to differences of site energies. They showed that approximating $G_{b}\left(\mathbf{R}_{b}\right)-G_{a}\left(\mathbf{R}_{a}\right)$ as the difference of the eigenvalues of the involved frontier orbitals yields a distribution consistent with a Gaussian model of site energies. Introduced by Bässler already in $1981^{105}$ the latter simply assumes a Gaussian distribution of site energies, based on the observation that absorption spectra in disordered organic solids are of Gaussian shapes with roughly similar widths. ${ }^{106}$

\subsection{Reorganization free energy, $\lambda$}

The reorganization free energy occurring in polaron hopping rate theories is in general a longrange property describing the electrostatic re- 
sponse of the surrounding medium to changes in the charge state of an active region, such as in a charge transfer between two neighboring sites. This long-range nature of $\lambda$ is generally hard to capture with electronic structure calculations due to the computational cost of simulating appropriately sized systems. A common way around this problem is to separate short-range - so called inner-sphere - and longrange, outer-sphere contributions to the reorganization free energy $\lambda=\lambda_{\text {in }}+\lambda_{\text {out }}$.

In organic solids, a natural separation is for example to designate the actual donor and acceptor molecules as inner-sphere and everything else as outer-sphere. The advantage of such a distinction is then that $\lambda_{\text {out }}$ can for instance be estimated in a mean-field approach via the Marcus continuum formula ${ }^{89}$

$$
\lambda_{\text {out }}=(\Delta q)^{2}\left(\frac{1}{\varepsilon_{\mathrm{op}}}-\frac{1}{\varepsilon_{\mathrm{s}}}\right)\left(\frac{1}{2 r_{a}}+\frac{1}{2 r_{b}}-\frac{1}{d_{a b}}\right) \text {. }
$$

Here, $\Delta q$ is the charge transferred, while $r_{\text {a }}$ and $r_{\mathrm{b}}$ are the effective radii of the donor molecule at site $a$ and the acceptor molecule at site $b$, respectively. $d_{a b}=\left|\mathbf{d}_{a b}\right|$ is the distance between the two sites as before, and finally, $\varepsilon_{\text {op }}$ and $\varepsilon_{\mathrm{s}}$ denote the optical (high frequency) and static dielectric permittivities in order to account for the electronic and nuclear response of the medium outside of the donor and acceptor cavities. The concept of effective radii is based on a simple continuum solvation model ${ }^{107}$ and originally $r_{a}$ and $r_{b}$ were introduced by Marcus as simple ionic radii in solution. While there is no unique definition of the radii, ${ }^{108-110}$ a common approximation is to assume the reactants are in close contact yielding $r_{a}+r_{b}=d_{a b},{ }^{111,112}$ which for identical molecular sites leads to $r_{a}+r_{b}=2 r_{a}=d_{a b}$. This implies that the effective radii can be approximated straightforwardly from lattice parameters of the molecular crystal.

The inner-sphere contribution is correspondingly given by the intra-molecular reorganization energies of donor and acceptor. It is given by $^{110} \lambda_{\text {in }}=G_{b}\left(\mathbf{R}_{a}\right)-G_{a}\left(\mathbf{R}_{a}\right)$, where $G_{a}$ and $G_{b}$ are the free energies of the state with the charge at site $a$ and $b$, respectively, while $\mathbf{R}_{a}$ and $\mathbf{R}_{b}$ are the corresponding equilibrium nuclear configurations (cf. Fig. 4). Given an electronic structure method that can give an estimate of these diabatic state energies $G_{a}$ and $G_{b}$ such as e.g. the constrained density functional theory method described in Section 3.7.2 below, $\lambda_{\text {in }}$ is straightforward to calculate. Yet, it generally involves an appropriate averaging over all nuclear degrees of freedom for instance through MD simulations. ${ }^{92}$ Otherwise, the standard way to approximate the inner-sphere reorganization free energy contribution is through the so-called 4-point scheme, where the system is separated into donor and acceptor fragments (representing sites $a$ and $b$ ). Denoting by $E_{\text {tot }}^{\mathrm{C}}$ and $E_{\text {tot }}^{\mathrm{N}}$ the total energies of charged and neutral donor and acceptor fragments, respectively, and by $\mathbf{R}_{\mathrm{C}}$ and $\mathbf{R}_{\mathrm{N}}$ the equilibrium geometries adopted by the charged and neutral fragments, the scheme reads

$$
\begin{aligned}
\lambda_{\text {in }} & =\left[E_{\text {tot }}^{\mathrm{C}}\left(\mathbf{R}_{\mathrm{N}}\right)+E_{\text {tot }}^{\mathrm{N}}\left(\mathbf{R}_{\mathrm{C}}\right)\right] \\
& -\left[E_{\text {tot }}^{\mathrm{C}}\left(\mathbf{R}_{\mathrm{C}}\right)+E_{\text {tot }}^{\mathrm{N}}\left(\mathbf{R}_{\mathrm{N}}\right)\right] .
\end{aligned}
$$

Observing again that the reorganization free energy is due to the change in nuclear coordinates $\mathbf{R}_{a} \rightarrow \mathbf{R}_{b}$ upon transfer of the charge from site $a$ to site $b$, another way to determine the full reorganization free energy $\lambda$ presents itself in the fact that each such displacement can be expressed-within a harmonic approximation - in terms of the normal modes (or phonons) of the respective system. By projecting the displacement $\Delta \mathbf{R}=\mathbf{R}_{a}-\mathbf{R}_{b}$ onto normal coordinates it is possible to determine the contribution of each normal mode $j$ to the reorganization energy. ${ }^{113}$ Denoting the $3 N$ vector of mass-weighted Cartesian displacement coordinates of a mode $v$ in state $\{a, b\}$ by $\mathbf{L}_{v}^{\{a, b\}}$, we can express this projection in terms of dimensionless displacement parameters

$$
B_{v}=\sqrt{\frac{\nu_{v}}{h}}(\Delta \mathbf{R})^{\mathrm{T}} \mathbf{M}^{1 / 2} \cdot \mathbf{L}_{v}^{a},
$$

where $M$ is the diagonal matrix of atomic masses associated with the $3 N$ Cartesian degrees of freedom and $\nu_{v}$ is the eigenfrequency of mode $v$. The weight of the contribution of each mode 
to the reorganization energy is then given by the dimensionless so-called Huang-Rhys factors $S_{v}=\frac{1}{2} B_{v}^{2}, 9,114$

$$
\lambda=\sum_{v} h \nu_{v} S_{v}
$$

where the sum extends over all $(3 N-6)$ modes. While within the harmonic approximation this is in principle exact for both inner and outer sphere contribution to $\lambda$, so far this approach has been mostly used for the inner-sphere reorganization energy. ${ }^{9,115-117}$ In this case the displacement projection is restricted to intramolecular modes, with donor and acceptor modes treated separately. Note that the harmonic approximation is thereby expected to work well for rigid molecules with small $\lambda$, but may break down for floppy molecules with potentially large deviations from their equilibrium geometries. ${ }^{118}$

Finally, a related approach to determine the reorganization energy is to perform an MD simulation in one of the charge transfer states to calculate the spectral density function $J(\omega)$ of the energy gap coordinate $(\Delta E)$ as defined by eq. $(12)^{110,119}$

$$
J(\omega)=\frac{\beta \omega}{2} \int_{0}^{\infty} d t\langle\delta \Delta E(0) \delta \Delta E(t)\rangle \cos \omega t .
$$

The straightforward generalization of eq. (26) to a continuum of vibrational states is then given by the integral over $J(\omega)$

$$
\lambda=\frac{2}{\pi} \int_{0}^{\infty} d \omega \frac{J(\omega)}{\omega} .
$$

\subsection{Nuclear tunneling factor, $\Gamma_{n}$}

The above expressions for the nuclear reorganization energy $\lambda$, equations $(23),(24),(26)$, and (28) all treat the nuclear degrees of freedom classically, which at low temperatures can lead to an overestimation of the reorganization barrier and thus an under-estimation of the hopping rate. This shortcoming is addressed through the introduction of a nuclear enhancement tunneling factor $\Gamma_{n}$ into eq. (13). Although most room temperature studies set
$\Gamma_{n}=1$ as long as the differences between the two polaronic geometries $a$ and $b$ are not extremely large, ${ }^{81,119}$ it is important to note here that in the case of organic semiconductors the comparatively large vibrational energy stored e.g. in $C=C$ bonds can lead to significant nuclear quantum effects. ${ }^{13}$ While tests e.g. on biological systems showed an enhancement factor of around two even for comparatively large vibrational energies, ${ }^{119}$ much lower reorganization energies in organic semiconductors compared to biological systems $(\approx 0.1 \mathrm{eV}$ vs $\approx 1 \mathrm{eV})^{119}$ can lead to a very different behavior. Thus, we here give a brief introduction into one of the main approaches available to determine the nuclear tunneling factor.

Given a fully quantum mechanical description

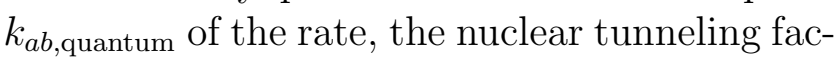
tor is simply defined as the ratio of the rate at the desired temperature $T$ and its high temperature $(T \rightarrow \infty)$ classical limit: ${ }^{7,81}$

$$
\Gamma_{n}(T)=\frac{k_{a b, \text { quantum }}(T)}{k_{a b, \text { quantum }}(T \rightarrow \infty)}
$$

A number of groups have put forward approximations to the full quantum rate expression ${ }^{120,121}$ based, among others, on Fermi's golden rule. Appealingly, the model pioneered by Holstein, ${ }^{122-124}$ instead yields a closed form expression of the tunneling factor ${ }^{81}$

$$
\begin{aligned}
\Gamma_{n}= & \left(\frac{\sinh \left(h \nu_{\text {in }} / 2 k_{\mathrm{B}} T\right)}{h \nu_{\text {in }} / 2 k_{\mathrm{B}} T}\right)^{1 / 2} \\
& \times e^{\left.-\left(\left(\lambda / h \nu_{\text {in }}\right) \tanh \left(h \nu_{\text {in }} / 4 k_{\mathrm{B}} T\right)\right)-\left(h \nu_{\text {in }} / 4 k_{\mathrm{B}} T\right)\right)} .
\end{aligned}
$$

Here, $h$ denotes Planck's constant, while $\nu_{\text {in }}$ is an effective frequency of the inner sphere contributions to the charge transfer mode. ${ }^{81}$ Note that only at large effective frequencies or low temperatures, $\Gamma_{n}$ will show significant deviation from unity and thus contributing noticeably to the charge transfer rate.

Finally, a related expression based on individual vibrational modes was recently given by 
Zhugayevych and Tretiak, ${ }^{13}$

$$
\Gamma_{n}=\exp \left[\sum_{v} S_{v}\left(\frac{\varepsilon_{v}}{2}-\tanh \frac{\varepsilon_{v}}{2}\right)\right],
$$

where $S_{v}$ and $\varepsilon$ again denote the Huang-Rhys factors and eigenenergies of the mode $v$, respectively. The advantage of eq. (31) over the effective treatment of eq. (30) is that it yields a more detailed insight into the contributions of each mode to the nuclear quantum effects.

\subsection{Effective frequency, $\nu_{\text {eff }}$}

The effective frequency contained in the LZ transition state expression, eq. (13), and the tunneling factor, eq. (30), describes the number of attempts to cross the barrier per unit of time. Within the LZ approximation, the barrier to charge transfer is largely determined through the nuclear reorganization. As such, $\nu_{\text {eff }}$ is then the (harmonic) vibrational frequency along the nuclear reaction coordinate connecting $\mathbf{R}_{a}$ and $\mathbf{R}_{b} \cdot{ }^{125}$

A straightforward way to compute $\nu_{\text {eff }}$ is again given in terms of eigenmodes of the system. As outlined above, the contribution of a given eigenmode $v$ to the overall nuclear reorganization is determined by the respective HuangRhys factor $S_{v}$. This factor can therefore also be used to weigh the contribution of each mode to the effective frequency ${ }^{125,126}$

$$
\nu_{\mathrm{eff}}=\sum_{v} \nu_{v} \frac{S_{v}}{\sum_{k} S_{k}} .
$$

If the projection of the reaction coordinate is dominated by a single normal mode $k$, i.e. $S_{v} \approx$ $\delta_{v k}$, the effective attempt frequency can thus be approximated as the frequency of the corresponding mode $\nu_{\text {eff }} \approx \nu_{k}$.

\subsection{Electronic coupling, $H_{a b}$}

As outlined above, $H_{a b}$ is defined as the electronic coupling between two diabatic states $\left|\psi_{a}\right\rangle$ and $\left|\psi_{b}\right\rangle$

$$
H_{a b}=\left\langle\psi_{a}|\hat{H}| \psi_{b}\right\rangle_{\mathrm{TS}}
$$

where $\hat{H}$ is the Hamiltonian of the system and the subscript TS again denotes taking the expectation value at the nuclear transition state. Due to the symmetry of the Hamiltonian matrix the order of initial and final state is irrelevant and thus $H_{a b}=H_{b a}$.

A successful computation of such coupling elements rests mostly on the way the diabatic wavefunctions are determined. These wavefunctions are not eigenfunctions of the Hamiltonian. Instead, they represent charge localized states that diagonalize the nuclear kinetic energy operator. ${ }^{127,128}$ Finding a transformation from the adiabatic eigenstates of the electronic Hamiltonian to such diabatic states is unfortunately not a trivial task, especially for extended systems ${ }^{129}$ where it generally may not even exist. ${ }^{130}$ The determination of $H_{a b}$ is therefore the most critical step in calculating polaronic hopping rates. A range of approaches approximating a diabatic electronic basis have been put forward in the literature. ${ }^{131}$ A key aspect to recognize in this respect is that the value of the electronic coupling element depends strongly on the orbital geometries of the respective diabatic wavefunctions, and as such also on the geometries of the involved donor and acceptor sites. It is of central importance to find diabatization methods that are both computationally efficient and accurate, especially in view of amorphous systems where calculations need to be carried out for many configurations. One commonly made assumption ${ }^{132,133}$ is an exponential distance dependence of electronic coupling. This is rationalized with an exponential decay of the diabatic wavefunction tails and allows for extrapolation of the coupling from just a few donor-acceptor distances.

As a further observation, we note that there is an exact relationship between diabatic and adiabatic state energies for a two-state donor/acceptor system. In this case, the energies of the two adiabatic states - labeled $E_{1}$ and $E_{2}$ in the following - are connected to the diabatic state energies $E_{a}$ and $E_{b}$ via the absolute 
value of the diabatic coupling as

$$
E_{1,2}=\frac{1}{2}\left(E_{a}+E_{b} \pm \sqrt{\left(E_{a}-E_{b}\right)^{2}+4\left|H_{a b}\right|^{2}}\right) .
$$

The above expression can simply be obtained by determining the characteristic polynomial of a two-state diabatic Hamiltonian matrix. A further-and highly utilized - simplification can be obtained for a symmetric problem such as a homo-molecular dimer, i.e. equivalent sites $a$ and $b$, where the energies of both diabatic states are exactly identical $E_{a}=E_{b}$. It is then easy to see that the excited state gap $\Delta E_{12}$ (cf. $\Delta^{\ddagger}$ in Fig. 4) is equal to twice the coupling electronic matrix element

$$
\Delta E_{12}=E_{2}-E_{1}=2\left|H_{a b}\right| .
$$

This implies that in symmetric cases the coupling can be calculated with any method that yields accurate adiabatic ground and excited state energies, $E_{1}$ and $E_{2}$. This fact was among others exploited by Brédas and co-workers for organic crystals to study the geometrical dependency of coupling matrix elements. ${ }^{134,135}$ Recently, in the context of DFT-based calculations, e.g. fully eigenvalue self consistent $\mathrm{GW}^{136}$ has been shown to yield excellent results for the ground and excited state properties of organic crystals. Unfortunately, for asymmetric cases no such simple correspondence exists and some form of diabatization is needed for the calculation of electronic couplings.

Over the years a large number of methods aimed at calculating electronic coupling elements has been published. ${ }^{131,137}$ They differ in several aspects, such as the construction of the Hamiltonian (wavefunction vs DFT vs semi-empirical approaches), the definition of the diabatic states, or whether full determinants or only frontier orbitals are coupled. Especially in the field of quantum chemistry, wavefunction based diabatization methods have long been the focus of active research. Early examples of such approaches include the block diagonalization ${ }^{138}$ of the adiabatic Hamiltonian - discussed in Sec. 3.7.5 - the line integral approach, ${ }^{139,140}$ or the calculation of di- abatic states from molecular properties, ${ }^{141}$ such as e.g. dipole moments. ${ }^{142}$ These then led to more advanced methods such as the generalized Mulliken-Hush method ${ }^{129}$ (cf. Sec. 3.7.1), multi-step block diagonalization of complete active space self-consistent field (CASSCF) wavefunctions, ${ }^{143}$ and the construction of approximate, "regularized" diabatic states by removing only the leading nuclear kinetic coupling terms from an adiabatic representation. ${ }^{144}$ For the sake of brevity, we here describe in detail only some of the available methods, limiting the discussion to the most common approaches found in literature. Recent, more focused discussions of the merits and shortcomings of coupling element calculation methods can be found for example in references. ${ }^{133,145-147}$

\subsubsection{The generalized Mulliken-Hush method}

In the last twenty years the generalized Mulliken-Hush (GMH) method, as based on early work by Mulliken ${ }^{148}$ and Hush ${ }^{149,150}$ and put forward by Cave and Newton, ${ }^{129,151}$ has become the de-facto reference method for the determination of diabatic coupling elements. ${ }^{133,145}$ It is based on the assumption that diabatic states localized at different sites possess zero off-diagonal elements of the dipole moment matrix for ground- and first excited states. This is ultimately based ${ }^{142}$ on the simple observation that the transition dipole moment between ground- and excited states tends to vanish in the - charge delocalizedadiabatic picture but necessarily takes on a relatively large finite value for - charge localizeddiabatic states. At the TS both adiabatic states are degenerate - and thus "rotate into each other" in the terms of quantum chemistry ${ }^{142}$ which gives rise to a sharp change in the dipole moment functions of the adiabatic state. At the TS a transformation between adiabatic and diabatic states can thus be found by diagonalizing the adiabatic dipole matrix. ${ }^{142}$

In addition, only the components of each dipole matrix element in the direction defined by the difference between adiabatic dipole moments of the ground and excited states are con- 
sidered. For a two-state problem as considered here, one thus searches for the set of states diagonalizing the dipole moment matrix. The transformation to these states from the adiabatic ground and first excited states is then taken as the diabatic transformation matrix. Denoting by $Z_{u v}$ the elements of the dipole moment matrix for adiabatic states $u, v=1,2$, the diabatic coupling element is thus

$$
\left|H_{a b}^{\mathrm{GMH}}\right|=\frac{\left|Z_{12}\right|}{\sqrt{\left(Z_{11}-Z_{22}\right)^{2}+4\left|Z_{12}\right|^{2}}} \Delta E_{12} .
$$

Note that in the case of a symmetric donor/acceptor system $\left(Z_{11}=Z_{22}\right)$ this expression for the coupling reduces trivially to the exact value of half the ground/first excited state energy splitting of eq. (35). Also, in literature the dipole moment matrix is generally denoted by the letter $\mu$, which we avoid here in order to distinguish it from the carrier mobility.

The GMH method is thus applicable to every electronic structure method that yields an accurate excited state splitting and gives access to transition dipole moments of these states. It is thus most often used in conjunction with wavefunction based approaches or hybridlevel time-dependent density functional theory (TDDFT), both of which tend to be computationally rather demanding for large systems and extended solids as of interest here.

\subsubsection{Constrained density functional theory}

There are a number of formulations of constrained density functional theory (CDFT). They nevertheless all rest on the same idea of adding an external potential to the density functional theory (DFT) electronic Hamiltonian in order to restrict the self-consistent field (SCF) cycle to a certain pre-determined molecular reference charge distribution. In the context of charge transfer between a donor and an acceptor site, this allows for the generation of approximate diabatic states by forcing the transferred charge (electron or hole) to localize at certain atoms or groups of atoms that comprise the donor and acceptor groups, respectively. As pointed out e.g. by $\mathrm{Wu}$ and van Voorhis, ${ }^{152}$ this basic idea is almost as old as the field of computational electronic structure theory itself, with its first use for a small model system already in 1963 by Karplus and Mukherji. ${ }^{153}$ Later it was, among others, picked up by Dederichs, ${ }^{154}$ Akai, ${ }^{155} \mathrm{Hy}-$ bertsen, ${ }^{156}$ Zhang, ${ }^{157}$ Warshel ${ }^{158,159}$ and Behler et al. ${ }^{160,161}$ for first studies of defect states, magnetism and charge transfer reactions. Today, most modern applications of the CDFT approach for molecular systems are based on work by $\mathrm{Wu}$ and van Voorhis, ${ }^{152,162-164}$ who formulated CDFT in form of a scleronomic (i.e. not explicitly time-dependent) constraint on the charge (or spin) density which is added to the Kohn-Sham energy functional via a Lagrange multiplier. This new energy functional $F\left[\rho, V^{\mathrm{L}}\right]$, acting on an electron density $\rho$ then reads $^{92}$

$$
F\left[\rho, V^{\mathrm{L}}\right]=E[\rho]+V^{\mathrm{L}}\left(\int w(\mathbf{r}) \rho(\mathbf{r}) d \mathbf{r}-N_{\mathrm{c}}\right),
$$

where the first term denotes the unconstrained Kohn-Sham energy functional and the second term the constraint. There, $V^{\mathrm{L}}$ is the Lagrange multiplier regulating the strength of the external potential, $w(\mathbf{r})$ is the weight function defining the regions of space that the charge is constrained to, and $N_{\mathrm{c}}$ is the desired charge in the region defined by $w$. Note that the Lagrange multiplier can generally not be determined analytically, but has to be optimized separately in a loop on top of the normal density SCF cycle. Also, it is evident from eq. (37) that the final, self-consistent charge-constrained state will depend somewhat on the choice of the weight function. ${ }^{92}$ Yet, this at first severe drawback is alleviated greatly in the calculation of coupling elements (see below), which in practice show only a limited sensitivity to the weight function. ${ }^{165}$ Nevertheless, this problem is analogous to attributing electronic charges to atoms in molecules. In the case of DFT, many different definitions of such charges exist - e.g. by Mulliken, ${ }^{166}$ Hirshfeld ${ }^{167}$ or Bader ${ }^{168}$ to name only a few of the most popular ones. Among these, 
a real-space definition of $w(\mathbf{r})$, often based on a form of Hirshfeld charges, has emerged as the standard choice for the study of charge transfer in the diabatic picture, ${ }^{11,132,169}$ due to their very good accuracy, ${ }^{133,145}$ robustness, and basis set independence. ${ }^{170}$

For a constraint on the charge in a region $D$ of a system with $M$ atoms, the Hirshfeld weight function reads

$$
w(\mathbf{r})=\frac{\sum_{u \in D} \rho_{u}\left(\mathbf{r}-\mathbf{R}_{u}\right)}{\sum_{u=1}^{M} \rho_{u}\left(\mathbf{r}-\mathbf{R}_{u}\right)},
$$

where $\rho_{u}\left(\mathbf{r}-\mathbf{R}_{u}\right)$ is the non-interacting, sometimes also called pro-molecular, free atom density of atom $u$ on position $\mathbf{R}_{u}$. For charge transfer between a donor and an acceptor group the constraint can actually also be expressed as a constraint on the charge difference between the groups. ${ }^{92}$ This avoids the need to define absolute charges of the respective donor and acceptor groups. An example of a weight function for a charge transfer dimer of tetracene molecules in a tetracene crystal is shown in Fig. 7.

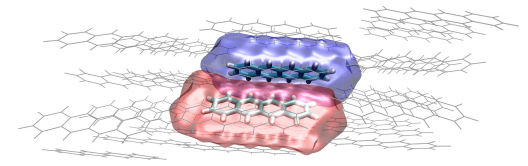

Figure 7: Hirshfeld weight function for a donor/acceptor pair of molecules in a tetracene crystal. Red shaded areas depict positive (charge accumulating) regions, blue areas negative (charge depleting) regions of the weight function. Shown in grey are surrounding molecules not directly included in the weight function.

Within the CDFT framework the diabatic state wavefunctions $\psi_{a}^{\mathrm{CDFT}}, \psi_{b}^{\mathrm{CDFT}}$ are approximated by those of Kohn-Sham eigenstates of suitably constrained DFT calculations with Hamiltonians $\mathcal{H}^{\mathrm{KS}}\left[\rho_{a}\right]+V_{a}^{\mathrm{L}} w_{a}$ and $\mathcal{H}^{\mathrm{KS}}\left[\rho_{b}\right]+$ $V_{b}^{\mathrm{L}} w_{b}$, respectively. The Hamiltonian transition matrix elements between both states can then in principle be determined as

$$
\begin{aligned}
H_{a b}^{\mathrm{CDFT}}= & \left\langle\psi_{a}^{\mathrm{CDFT}}\left|\mathcal{H}^{\mathrm{KS}}\left[\rho_{b}\right]\right| \psi_{b}^{\mathrm{CDFT}}\right\rangle \\
= & F_{b}\left\langle\psi_{a}^{\mathrm{CDFT}} \mid \psi_{b}^{\mathrm{CDFT}}\right\rangle \\
& -V_{b}^{\mathrm{L}}\left\langle\psi_{a}^{\mathrm{CDFT}}\left|w_{b}\right| \psi_{b}^{\mathrm{CDFT}}\right\rangle \\
H_{b a}^{\mathrm{CDFT}}= & \left\langle\psi_{b}^{\mathrm{CDFT}}\left|\mathcal{H}^{\mathrm{KS}}\left[\rho_{a}\right]\right| \psi_{a}^{\mathrm{CDFT}}\right\rangle \\
= & F_{a}\left\langle\psi_{b}^{\mathrm{CDFT}} \mid \psi_{a}^{\mathrm{CDFT}}\right\rangle \\
& -V_{a}^{\mathrm{L}}\left\langle\psi_{b}^{\mathrm{CDFT}}\left|w_{a}\right| \psi_{a}^{\mathrm{CDFT}}\right\rangle .
\end{aligned}
$$

Note that, in this formulation $H_{a b}^{\mathrm{CDFT}}$ would not be guaranteed to equal $H_{b a}^{\mathrm{CDFT}}$. This complicates the comparison of CDFT transition matrix elements to those determined with other methods or with experiment. Furthermore, the CDFT-generated diabatic reference wavefunctions are not generally orthogonal, as they are not eigenstates of the same Hamiltonian. States $\psi_{a}^{\mathrm{CDFT}}$ and $\psi_{b}^{\mathrm{CDFT}}$ generally have non-vanishing overlap $\left\langle\psi_{b}^{\mathrm{CDFT}} \mid \psi_{a}^{\mathrm{CDFT}}\right\rangle=S_{a b}^{\mathrm{CDFT}}$. This motivates a transformation as done in the GMH method (see Section 3.7.1), i.e. to arrive at the final diabatic states they are transformed such that the final wavefunctions $\psi_{a, b}$ diagonalize both their overlap matrix $\mathbb{S}=\left\langle\psi_{u} \mid \psi_{v}\right\rangle$ and the weight matrix $\mathbb{W}=\left\langle\psi_{u}\left|w_{v}\right| \psi_{v}\right\rangle$ with $u$ and $v \in\{a, b\}$. Matrix elements $H_{a b}=H_{b a}$ in the basis of the orthogonal states are then symmetric and can be compared to other methods.

In passing we note that an analogous formulation of CDFT for extended solid-state systems was put forward by Behler and co-workers. ${ }^{161} \mathrm{In}$ their work, the constraint potential is not determined via a Lagrange multiplier but rather via the equalization of the Fermi levels between the constraint regions. This nevertheless leads to equations very similar to those outlined above. On a final note we also point out that recently Rapacioli, Spiegelman et al. combined the CDFT approach with a self-consistent charge tight-binding formulation of DFT. ${ }^{171-173}$ In the latter method, the total energy functional is expanded to second order in a localized basis set, to ultimately yield a Hamiltonian matrix analogous to "full" DFT but with precalculated and tabulated elements for each atomic basis function. An in-depth review of DFTB methods can for example be found in refs. ${ }^{174-176}$ 


\subsubsection{Fragment orbital methods}

The fundamental assumption behind the fragment orbital (FO) family of methods is that only the frontier orbitals directly partaking in the charge transfer do change between initial and final diabatic state. ${ }^{177,178}$ All others remain unperturbed. To illustrate this assumption, we consider the transfer of an excess electron from a donor group to an acceptor group. Excluding the excess electron, the donor group has $M$ occupied orbitals while the acceptor has - in general $-N$ occupied orbitals. Denoting these single electron orbitals as $\phi_{\mathrm{D}, \mathrm{A}}^{i}$ for donor and acceptor, respectively, diabatic wavefunctions are given by

$$
\begin{aligned}
\psi_{a} & =\frac{1}{\sqrt{(M+N+1) !}} \\
& \times \operatorname{det}\left(\phi_{\mathrm{D}}^{1} \ldots \phi_{\mathrm{D}}^{M} \phi_{\mathrm{D}}^{M+1} \phi_{\mathrm{A}}^{1} \ldots \phi_{\mathrm{A}}^{N}\right) \\
\psi_{b} & =\frac{1}{\sqrt{(M+N+1) !}} \\
& \times \operatorname{det}\left(\phi_{\mathrm{D}}^{1} \ldots \phi_{\mathrm{D}}^{M} \phi_{\mathrm{A}}^{1} \ldots \phi_{\mathrm{A}}^{N} \phi_{\mathrm{A}}^{N+1}\right) .
\end{aligned}
$$

Under the above assumption of otherwise constant orbitals, the calculation of the transition matrix element between wavefunctions $\psi_{a}$ and $\psi_{b}$ reduces to determining the Kohn-Sham matrix element between the two single-electron frontier orbitals

$$
H_{a b}^{\mathrm{FO}-\mathrm{DFT}} \approx\left\langle\phi_{\mathrm{D}}^{N+1}\left|\mathcal{H}^{\mathrm{KS}}\right| \phi_{\mathrm{A}}^{M+1}\right\rangle .
$$

This implies that-given the knowledge of the effective single-particle Hamiltonian of the system - the matrix element only depends on orbitals which are localized on either the donor or the acceptor. In the case of inter-molecular charge transfer this gives rise to the idea of treating the donor and acceptor as fragments in two separate calculations and then combining their wavefunctions to determine $\mathcal{H}^{\mathrm{KS}}$ and the transition matrix element. This approach guarantees the localization of the diabatic wavefunctions on the respective fragments independent of any errors of the underlying method, such as the electron delocalization error of semilocal DFT. ${ }^{95}$ On the other hand, in separating donor and acceptor fragments any direct influ- ences between them - predominantly polarization of one fragment through the charge density on the other one - are neglected. Furthermore, the way how the full Hamiltonian is constructed from the fragment wavefunctions gives rise to a number of different "flavors" of the FO-DFT method. ${ }^{16}$ Specifically this concerns the number of electrons used to construct the reference donor and acceptor states, as well as $\mathcal{H}^{\mathrm{KS}}$.

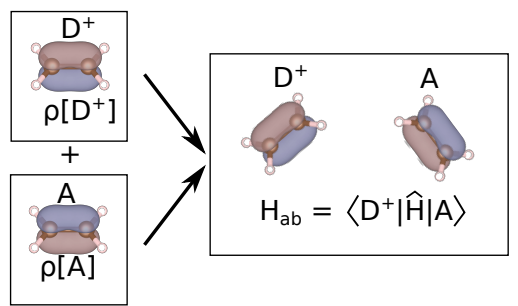

Figure 8: Sketch of the fragment orbital DFT approach for hole transfer across an ethylene dimer. Both fragments, donor and acceptor, are treated separately when determining their SCF ground state wavefunctions and densities $\rho\left[\left\{\mathrm{D}, \mathrm{A}^{+}\right\}\right]$. These are then used to construct a Hamiltonian of the combined system, with the wavefunctions acting as reference orbitals for the diabatic state.

In the original formulation of the method by Senthilkumar and co-workers, ${ }^{177}$ regardless of the actual charge transferred, the Hamiltonian is always constructed from the orbitals of the neutral fragments and the reference states are taken as the lowest unoccupied molecular orbitals (LUMO) in the case of electron transfer (ET) and the highest occupied molecular orbitals (HOMO) in case of hole transfer (HT). In this approach the Hamiltonian is therefore constructed from a charge density that deviates from that of the real combined system by exactly the charge that is initially on the donor and that is actually transferred, $\mathcal{H}^{\mathrm{KS}}=$ $\mathcal{H}^{\mathrm{KS}}[\rho(M+N)]$. Computationally, this holds the advantage that only neutral fragments need to be calculated, but can lead to systematic deviations of the matrix elements due to the deviating number of electrons. ${ }^{133,145}$

Another approach is therefore to treat both fragments in the reduced state $(M+N$ electrons in the case of $\mathrm{HT}$ and $M+N+2$ for ET) in order to determine the reference states and to con- 
struct $H^{\mathrm{KS}}$ from these orbitals with the occupation number of one of the frontier orbitals set to zero $\left(\phi_{\mathrm{D}}^{N}\right.$ and $\phi_{\mathrm{A}}^{M}$ for HT states $\psi_{\mathrm{a}}$ and $\psi_{\mathrm{b}}$, respectively, and $\phi_{\mathrm{D}}^{N+1}$ and $\phi_{\mathrm{A}}^{M+1}$ for ET states $\psi_{a}$ and $\psi_{b}$, respectively). ${ }^{37,132}$ This approach guarantees the correct number of electrons in the effective Hamiltonian $\mathcal{H}^{\mathrm{KS}}=\mathcal{H}^{\mathrm{KS}}[\rho(M+N-c)]$, where $c=+1$ for electron transfer and $c=-1$ for hole transfer. In non-symmetric systems this creates in principle two Hamiltonians that differ by the fragment orbital the electron was removed from, in close analogy to the situation in the CDFT approach. Due to numerical inaccuracies this can in turn lead to differences between the two matrix elements $H_{a b}$ and $H_{b a}$ and thus a non-Hermitian diabatic Hamiltonian. Standard practice in such cases is to average over the two off-diagonal elements, which amounts to a symmetrization of the two-state Hamiltonian. This approach does, however, neglect (HT) or overestimate (ET) charge reorganization within the respective fragments due to the excess charge. ${ }^{179}$

A most recent version of FO-DFT ${ }^{179}$ does also consider these intra-fragment effects of the excess charge by constructing the reference state from a charged and an uncharged fragment, e.g. $\psi_{\mathrm{D}}^{M+c}$ and $\psi_{\mathrm{A}}^{N}$ as illustrated in Fig. 8. The Hamiltonian constructed this way not only has the correct overall number of electrons but also directly includes orbital relaxations due to the excess charge. This on the other hand, comes at the price of more approximate reference orbitals. All three of these "flavors" of FO-DFT have been compared and critically discussed in a recent study by Schober and co-workers. ${ }^{179}$ In this work results showed a clear advantage of the latter charged fragment-orbital procedure, potentially yielding much greater accuracy than "standard" FO-DFT approaches and at very little additional computational cost due to the need to calculate both charged and neutral fragments. As a sidenote, Baumeier et al. ${ }^{180}$ as well as Sutton et al. ${ }^{181}$ recently demonstrated in their exhaustive studies of density functionals, the functional dependency of FO-DFT calculated coupling values for organic semiconductors. The latter demonstrated a near linear correlation of $H_{a b}$ with the amount of exact exchange in the functional, ultimately scaling the coupling by almost a factor of two from GGA functionals to pure Hartree-Fock calculations. This further underlines the need to carefully benchmark calculated coupling elements against available test-data. ${ }^{133,145}$

Finally, it is important to note that, although successfully applied by a number of groups, ${ }^{16,104,133,145,177,179,182}$ fragment orbital approaches are not all restricted to the use with DFT. Indeed any electronic structure method that allows for a combination of fragment wavefunctions and gives access to a combined Hamiltonian would be suitable. Elstner, Kubař and co-workers, for instance, presented a series of studies based on a DFT tight-binding (see above) formulation of the fragment orbital approach (FO-DFTB). ${ }^{176}$ The early research focus there lay on charge transport in DNA ${ }^{183-185}$ and bio-molecular poly-peptides. ${ }^{186,187}$ Most recently though the FO-DFTB approach was also applied to CT in organic semiconductors. ${ }^{188,189}$ There it was also demonstrated that environmental effects can straightforwardly be incorporated into FO calculations, e.g. via quantum mechanical/molecular mechanical embedding approaches. ${ }^{176,190}$ Concerning the accuracy of this approach, recent benchmark studies ${ }^{133,145}$ indicated relative errors of FO-DFTB matrix elements to be $\sim 10-20 \%$ larger than that of the respective FO-DFT flavors, yet, of course, at much reduced computational cost.

Even more economical variants of the FO scheme are based on semi-empirical electronic structure methods such as Zerner's independent neglect of differential overlap (ZINDO). ${ }^{191}$ In this method, the Hamiltonian is also constructed in a localized minimal basis, yet with empirically determined parameters. Kirkpatrick and co-workers showed ${ }^{27,192,193}$ the feasibility of such an approach even for very large systems. Finally, Akimov ${ }^{194}$ recently employed a fragment orbital scheme based on extended Hückel theory to determine electronic couplings for use in the non-adiabatic propagation of charge carriers (cf. sec. 5.3). 


\subsubsection{Frozen density embedding}

The frozen density embedding (FDE) ansatz, initially presented by Wesolowski and Warshel ${ }^{158}$ and later adapted to electronic coupling calculations by Pavanello and Neugebauer, ${ }^{195}$ rests on the partition of a large, but non-covalently bound system into smaller fragments. The total density of the system is thereby expressed as a sum over the number $N_{\mathrm{S}}$ of sub-system densities $\rho_{w}(r)$

$$
\rho(r)=\sum_{w=1}^{N_{\mathrm{S}}} \rho_{w}(r) .
$$

The main difference to FO-DFT is that the fragments do interact with each other via their Kohn-Sham effective potentials. In essence, for each fragment a DFT SCF cycle is performed including a constant embedding potential described as the sum of the Kohn-Sham potentials of all other fragments. This in turn yields a new effective potential per fragment. This change in potential has to be accounted for in an outer SCF cycle for each fragment to respond to the presence of the others fragment. ${ }^{196,197}$ As an illustration of this approach we depict in Fig. 9 the local part of a FDE potential for a cluster of tetracene molecules.

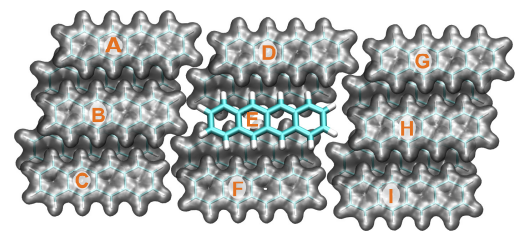

Figure 9: Illustration of the local part of the iso-surface of the embedding potential of a cluster of tetracene molecules acting on a central molecule (calculated with DFT-PBE ${ }^{198}$ and using the FHI-aims code ${ }^{199}$ ). In an FDE calculation, the embedding potential is constructed as a superposition of the potentials of each participating fragment $\{\mathrm{A}-\mathrm{D}, \mathrm{F}-\mathrm{I}\}$, with one of the fragments treated explicitly $\{\mathrm{E}\}$. Density and potential of the central fragment $\mathrm{E}$ would then be determined by treating the embedding potential as fixed, to be used in the determination of the embedding potential of the other fragments in an outer SCF cycle.
Initially this ansatz was used to efficiently determine DFT energies of large super-systems. It was thereby noted early on that the FDE method naturally suppresses the semilocal DFT electron delocalization error and yields fragment-localized charge states similar to the diabatic states of electron transfer. ${ }^{200-202}$ Pavanello and co-workers have pointed out ${ }^{146,203,204}$ that this is mostly due to the missing orthogonality between orbitals of different fragments, the localized initial guess, basis sets which effectively rule out delocalization of orbitals over more than one fragment, as well as effective repulsive regions near the frozen densities of other fragments.

Having constructed the diabatic reference states one could in principle proceed similarly to the FO-DFT or CDFT methods by determining the combined system's Hamiltonian and extracting the respective matrix elements. The approach chosen by Pavanello ${ }^{195,203,205}$ instead follows a slightly different path, observing that the coupling between (non-orthogonal) diabatic states $a$ and $b$ can be approximated as

$$
H_{a b} \approx S_{a b} E\left[\rho^{(a b)}(\mathbf{r})\right],
$$

where $S_{a b}$ is the overlap between the two KohnSham determinants of $a$ and $b, E\left[\rho^{(a b)}(\mathbf{r})\right]$ denotes an energy functional of a transition density

$$
\rho^{(a b)}(\mathbf{r})=\sum_{u v}^{\text {occ }} \phi_{u}^{(a)}(\mathbf{r})\left(\mathbb{S}^{(a b)}\right)_{u v}^{-1} \phi_{v}^{(b)}(\mathbf{r}),
$$

and the sum goes over all occupied states. The overlap matrix between all orbitals contributing to $a$ and $b$ is denoted by $\mathbb{S}^{(a b)}$. This approximation can be seen as motivated by the solution of a generalized eigenvalue problem for the - generally unknown - diabatic Hamiltonian. ${ }^{206}$ In order to yield a coupling element comparable to other methods, the final step in this procedure is an orthogonalization step of 
the resulting diabatic Hamiltonian matrix via

$$
H_{a b}^{\mathrm{FDE}}=\frac{1}{1-S_{a b}^{2}}\left(H_{a b}-S_{a b} \frac{H_{a a}+H_{b b}}{2}\right) .
$$

The energy functional $E\left[\rho^{(a b)}(\mathbf{r})\right]$ appearing in eq. (43a) in principle denotes an appropriate energy functional of the full system evaluated for the transition density $\rho^{(a b)}(\mathbf{r})$ given in eq. (43b). ${ }^{203}$ Yet, it can also be calculated from fragments according to the frozen density embedding scheme.

\subsubsection{Block diagonalization of the elec- tronic Hamiltonian}

Based on similar ideas as the fragment orbital method, block diagonalization (BD) ${ }^{138,143,151}$ approximates the coupling between the two diabatic wavefunctions by the off-diagonal elements of a reference Hamiltonian in a localized basis. Initially conceived for wavefunctionbased electronic structure methods, the approach has found use with DFT in the context of extended systems. ${ }^{207}$ We will therefore illustrate it in terms of a Kohn-Sham DFT Hamiltonian, but keeping in mind that the method can equally be used with Hartree-Fock, single- or multi-reference wavefunction based approaches. The basic principle of the BD method is to transform the Hamiltonian matrix from a localized atomic basis into a basis of eigenstates of fragments of the complete system. These fragments are chosen analogously to the FODFT ansatz but, as will be seen below, can also be covalently bonded to other fragments. The basis of fragment eigenstates is found by separate diagonalization of the Hamiltonian submatrices belonging to each of the fragments, where the fragments are identified by their respective localized basis functions. Within these eigenspaces one can then determine the frontier orbitals by counting the states up to the HOMO and LUMO orbitals of the fragments. The unitary transformation $\mathbb{U}$ matrix of the complete Hamiltonian is thereafter simply given as a combination of the eigenvectors $\mathbb{V}_{a, b}$ of the fragments $a$ and $b$

$$
\mathbb{U}=\left(\begin{array}{cc}
\mathbb{V}_{a} & 0 \\
0 & \mathbb{V}_{b}
\end{array}\right)
$$

The electronic coupling $H_{a b}$ is finally extracted from the thus transformed Hamiltonian as the matrix element between the respective frontier orbitals as illustrated in Fig. 10.

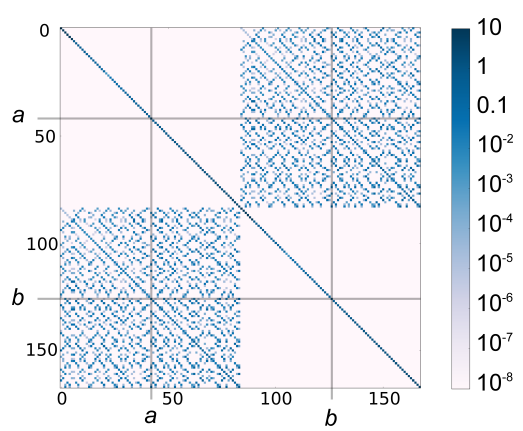

Figure 10: Demonstration of the blockdiagonalization method for a benzene dimer (calculated with DFT-PBE ${ }^{198}$ and using the FHI-aims code ${ }^{199}$ ). Shown is an orthogonalized and block diagonalized Hamiltonian matrix, for illustrative purposes in a logarithmic scale of the absolute values. Elements belonging to the approximated HOMO orbitals on molecules a and $b$ are highlighted by grey lines.

Unfortunately, this method does suffer from some ambiguity when comparing to other methods, since the localized basis functions of the respective fragments generally show nonvanishing overlap with each other. This means that in order to obtain meaningful Hamiltonian matrix elements ${ }^{208-210}$ it is advantageous to introduce a basis set orthogonalization step, e.g. employing the symmetric method of Löwdin 211,212

$$
\begin{gathered}
\mathscr{H}_{\text {ortho }}=\mathbb{X}^{T} \amalg \mathbb{X} \\
\mathbb{X}=\mathbb{S}^{-1 / 2},
\end{gathered}
$$

where $\mathbb{S}$ denotes the overlap matrix. Complications arise here, because any orthogonalization scheme necessarily mixes the basis functions and thus potentially blurs the clear separation between donor and acceptor fragment orbitals. Yet, as e.g. pointed out by Thoss and co-workers, ${ }^{207}$ the symmetric approach has 
two advantages. First, it is least biased compared to other methods, such as for example the Gram-Schmidt scheme, where all basis functions are orthogonalized with respect to one unchanged reference basis function. ${ }^{212,213}$ Second, the Löwdin method preserves the character of the basis in a least squares sense such that its localization is mostly retained and thus the separation between fragments is mostly upheld. ${ }^{214}$ Yet, there are still systems where the donor/acceptor separation fails at this stage, especially in the case of larger basis sets necessary for instance to represent unoccupied orbitals. ${ }^{215}$ Careful consideration of the resulting reference orbitals is therefore generally necessary.

\subsubsection{Analytic overlap method}

The methods discussed so far, while reasonably accurate and significantly cheaper than quantum chemical reference methods, still require at least one DFT calculation per geometry. In the case of static, rate-based approaches a single-point DFT calculation per donor/acceptor pair is in general computationally affordable, even in systems with a large number of local donor/acceptor configurations. ${ }^{37}$ Yet, there are cases where even such comparatively undemanding approaches become unfeasible. An example would be a direct propagation of the excess charge in a non-adiabatic MD scheme as discussed in Section 5.3 below. There, or in the case of snapshot sampling for disordered systems, a rapid on-the-fly estimation of $H_{a b}$ is essential. In such cases one needs to resort to semi-empirical approaches, for instance FO-DFTB where relevant integrals are tabulated and readily available for a given molecular geometry. An even simpler way to rapid estimation of electronic couplings was recently suggested by Gajdos et al. ${ }^{216}$ Their analytic overlap method (AOM) rests on the idea of establishing a correlation between the electronic coupling and a descriptor that can be very rapidly calculated for a given donor-acceptor geometry. A good linear correlation between the electronic coupling and singly occupied molecular orbital (SOMO) overlap $S_{a b}^{\mathrm{SOMO}}$ was for instance identified. This is analogous to resonance integral calculations in extended Hueckel theory, which only differ in the type of reference state used in place of the atomic orbitals. Furthermore, Gajdos et al. approximated the overlap integral through an analytical expression, by projecting the frontier orbitals of a reference DFT calculation onto Slater-type $p$-orbitals. This way, for not too radical departures from the molecular reference geometry, $S_{a b}^{\mathrm{SOMO}}$ can be estimated without any further DFT calculations for the different geometries. For example, along an MD-trajectory the direction of the atomic $p$-orbital basis functions that form a given fragment $\pi$-orbital are updated at each nuclear time step. This is in a first approximation performed simply by enforcing the $p$-orbital axis to lie perpendicular to the $\pi$-conjugated plane defined by the atom it is centered on and its neighboring atoms.

In the AOM approximation the Hamiltonian matrix element reads

$$
H_{a b}^{\mathrm{AOM}}=C S_{a b}^{\mathrm{SOMO}}
$$

where $C$ is a constant of proportion obtained by a linear fit to $H_{a b}$ values from explicit electronic structure calculations. For a training set of small organic molecules Gajdos et al. determined $C$ to be $1.819 \mathrm{eV}$. The same linear correlation applied remarkably well to a test set of $\pi$-conjugated molecules not included in the calibration. With a view on dynamical trajectories, the authors further tested the importance of updating the $p$-orbital expansion coefficient at each nuclear time step and found that it can be neglected for rigid molecules. ${ }^{93}$ However, for larger molecules with soft intramolecular modes (like e.g. polymers) the fragment orbitals may undergo large electronic reorganizations as the nuclei evolve. In this case one could carry out an ensemble of reference electronic structure calculations for important conformations and use on-the-fly interpolation methods to reconstruct the fragment orbital during the MD simulation.

Due to the fact that this method rests solely on analytical terms and just a single reference DFT calculation per molecule is required, its speed easily surpasses any other method on the 
current list, while nevertheless reaching comparable accuracies. While Gajdos et al. observed a remarkably good linear correlation for a wide range of $\pi$-conjugated compounds, it may be advantageous (and straightforward) to reparametrize the linear relation for a given molecule under consideration for a further refinement of the coupling value estimation. The constant of proportionality is thereby very sensitive to the exponential decay of the frontier orbitals. Hence, application of AOM to ET between non-organic/non $\pi$-conjugated donor acceptor systems would require parametrization of the relation.

\subsubsection{Super-exchange effective cou- plings}

While not a diabatization method, we consider here also the case of effective couplings for super-exchange (SE) charge transport. All previously mentioned approaches considered direct $\mathrm{ET}$ between donor and acceptor with the space between them being empty (vacuum). If the space between donor and acceptor is made up of molecules or a covalent bridge connecting them, most of these methods can still be used to compute couplings. However, the values quickly become very small with increasing bridge length. Here the SE model for ET can be used to estimate effective electronic couplings. In this method it is assumed that the ET is mediated by the lowest unoccupied and/or highest occupied orbitals of the bridge. These bridging orbitals possess considerably different site energies $\left(\Delta G^{0} \neq 0\right)$ even without external electric field, or they could simply be considered as other diabatic states.

Examples for SE can be found in monocrystals bearing point defects, among different molecules in heterocrystals, or in disordered systems. ${ }^{217}$ In the SE model the effective coupling between donor and acceptor sites can be effectively be expressed using the Green's function of the bridge ${ }^{35}$

$$
\mathbb{G}^{\mathrm{B}}(E)=\left(E \mathbb{S}^{\mathrm{B}}-\mathfrak{H}^{\mathrm{B}}\right)^{-1},
$$

where $\mathbb{S}^{\mathrm{B}}$ and $\mathbb{H}^{\mathrm{B}}$ are overlap and Hamiltonian matrices of the bridge orbitals, and $E$ is the tunneling energy, which is generally taken as equal to the energy at the transition state between initial and final state. ${ }^{14,218}$ The bridge-mediated effective coupling element is then given as a sum over all bridge states $N^{\mathrm{B}}$

$$
H_{a b}^{(\mathrm{SE})}=\sum_{u, v=1}^{N^{\mathrm{B}}}\left(H_{a u}-E S_{a u}\right) G_{u v}^{\mathrm{B}}\left(H_{b v}-E S_{b v}\right)
$$

Here, $H_{a u}$ and $H_{b v}$ are the coupling elements between the bridge states and the donor and acceptor orbitals, respectively, while $S_{a u}$ and $S_{b v}$ are the respective overlap matrix elements.

While super-exchange models have so far been mostly applied in bio-chemical simulations, ${ }^{14,219,220}$ first applications in organic solids recently started to emerge, ${ }^{217}$ and more can be expected as research moves towards more complex systems.

\subsection{Kinetic Monte Carlo simula- tion of $\mu$}

Initially developed to track radiation damage in solids ${ }^{221}$ the kinetic Monte Carlo (kMC) method evolved in the past 50 years into the main method to study the dynamical evolution of reaction networks connected by rare events. ${ }^{28,222,223}$ As already alluded to earlier, charge carrier hopping through a solid can be viewed as exactly such a process. ${ }^{27,91,193,224}$ This is illustrated in Fig. 11, where we depict the network of all possible nearest and next-nearest neighbor carrier hops in a naphtalene crystal. The basic assumption behind a hopping model is that carriers are localized on individual sites (here individual naphtalene molecules) and percolate as a series of discrete hops between sites $a$ and $b$ with a rate $k_{a b}$. Such a problem is ideally suited for a solution with $\mathrm{kMC}$. The advantage of a kMC approach over the analytical solution with eq. (11) is that it can also be applied to less regular systems, including for example lattice defects.

Among a variety of different, numerically efficient kMC algorithms, we will limit our discus- 


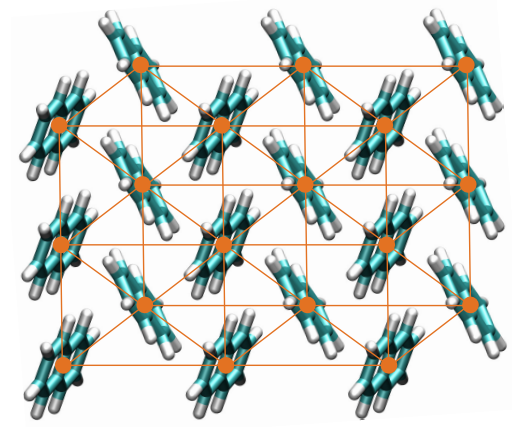

Figure 11: Illustration of a possible hopping network in a naphtalene crystal. Shown is a 2D cut of the crystal with each nearest and nextnearest neighbor sites connected to each other.

sion to the common, so-called "rejection free" procedure. ${ }^{225}$ In the case of just a single excess charge carrier moving through a system, thus disregarding any interaction between carriers, the corresponding $\mathrm{kMC}$ algorithm turns out to be surprisingly simple

1. At each kMC step with the carrier localized at site $a$, determine the site $b$ for the next hop from the pool of connected sites with a probability proportional to the rate $k_{a b}$ associated with this connection. Technically, this can be achieved by picking a random number $p$ between 0 and $\sum_{b} k_{a b}$, where the sum goes over all sites connected with non-zero rate to the current site $a$. The site $b$ to hop to is then determined by finding which interval

$$
p \in\left[\sum_{b^{\prime}=0}^{b} k_{a\left(b^{\prime}-1\right)}, \sum_{b^{\prime}=0}^{b+1} k_{a\left(b^{\prime}-1\right)}\right],
$$

the chosen random variable falls into.

2. Having picked the process $a \rightarrow b$ to execute, the simulation timer needs to be advanced. The waiting times $t$ for this process to occur are hereby exponentially distributed

$$
p_{a b}(t)=k_{a b} e^{k_{a} b t}
$$

Thus, the next step is to pick an exponentially distributed random variable to advance the simulation clock.
3. Having picked a process and a waiting time the final step in a $\mathrm{kMC}$ cycle is to move the carrier to the new site $b$ and return to step 1.

This $\mathrm{kMC}$ cycle is repeated either for a number of steps or until a given desired total simulation time $\tau$ is reached. The procedure hereby mimics charge diffusion trajectories through the molecular solid. In order to determine the mobility according to either eq. (4) or (6) one needs to determine either the average drift velocity or the root mean square displacement, respectively. This can generally be achieved by averaging over many created kMC trajectories. In the standard case of time-independent fields and bulk crystals this is typically replaced by a time-average over one sufficiently long kMC trajectory. For the average drift velocity one thereby considers that each carrier hop $a \rightarrow b$ in the trajectory covers the distance vector between the centers of mass $\mathbf{d}_{a b}$. For the Einstein-Smoluchowski mobility expression, eq. (6), these distances can be square-averaged and tabulated versus the corresponding waiting times $t$ to yield the component-resolved MSD and thus the diffusion matrix $D_{i j}$. For the general mobility equation, eq. (4), a field $\mathbf{E}$ can be applied, which corresponds to adjusting the hopping rates (eqs. (13-18), depending on which rate theory is considered). The drift velocity averaged over the whole simulation is then simply determined by the distance vector between the center of mass of the initial site for the excess charge $\mathbf{R}_{\text {initial }}$ and its final site $\mathbf{R}_{\text {final }}$-possibly taking care of periodic boundary conditions - and the overall simulation time $\tau$

$$
\langle\mathbf{v}\rangle=\frac{\mathbf{R}_{\text {final }}-\mathbf{R}_{\text {initial }}}{\tau} .
$$

As noted, this simplified approach applies only to the diffusion of a single excess charge carrier, or equivalently to a negligible interaction between excess charge carriers. This approximation holds typically only for very dilute carrier concentrations. For higher carrier densities one generally needs to include interactions with other carriers to achieve a faithful representation of carrier diffusion. ${ }^{226}$ Prominently this concerns the "blocking" of sites by carriers 
or finite Coulomb interactions between carriers localized at neighboring sites. The practical treatment of such lateral interactions in $\mathrm{kMC}$ simulations is still very much subject of active research and we refer the interested reader to ${ }^{227-231}$ and references therein for a discussion of present state-of-the-art approaches.

\subsection{Applications}

The use and value, but also the limitations of hopping models in the context of charge transport in organic crystals is documented in a wide range of literature. $\mathrm{C}_{60}$ and other fullerene derivatives are certainly among the most thoroughly studied molecular solids of the last decade. In 2009 Nelson and co-workers ${ }^{193}$ employed a Marcus-hopping model, based on semi-empirical electronic structure calculations, to study the grain-size dependence of the mobility in disordered $\mathrm{C}_{60}$ films. In good agreement to experiment, their simulations showed only a slight dependence of the mobility on the grain size. The same group later studied the effect of $\mathrm{C}_{60}$ functionalization on packing order and thus mobility. ${ }^{26}$ The actual validity of the hopping model in general and the specific hopping regimes (Section 3.1) in particular, was only subsequently scrutinized by some of us, first for unmodified $\mathrm{C}_{60}$ fullerenes ${ }^{37}$ and later for the popular PCBM modification. ${ }^{38}$ In these DFT-based studies outliers with large coupling elements did cast doubt on the general applicability of the hopping model in these systems. On the other hand, experimental mobilities could still be reproduced to within the correct order of magnitude. In a similar approach, Idé and co-workers ${ }^{232}$ studied the mobility of crystalline fullerene derivatives-PCBM and 1-(3-methoxycarbonyl)propyl-1-thienyl-[6,6]methanofullerene (ThCBM) - in a hoppingbased $\mathrm{kMC}$ model to estimate the influence of chemical modification on the fullerenes' transport properties.

With regards to other molecules, Di Motta et al. ${ }^{233}$ estimated charge diffusion constants for benzoquinone derivatives - 2,3dichloro-5,6-dicyano-1,4-benzoquinone(DDQ), tetrachlorobenzo-1,4-quinone (TCQ), and tetracyanoquinodimethane (TCN) as well as pentacene - to establish their suitability as resistive molecular memories. A similar approach was recently followed by Huong, Tai and Nguyen for modified thiophene compounds. ${ }^{234}$ McMahon and Troisi ${ }^{235}$ evaluated the reorganization free energies of several polyacenes as a measure for their hopping mobility, and Cornil and co-workers employed hopping models to study interfacial dielectric layers of pentacene on polystyrene and polymethacrylate. ${ }^{236}$

Yet, application of hopping models is not solely confined to ordered molecular crystals. For example, Friederich et al. ${ }^{99}$ recently published a study of disorder effects of amorphous materials composed of coordination complexes (tris(8-hydroxyquinolinato)aluminium) and molecules (N,N'-di(1-naphthyl)-N, $\mathrm{N}^{\prime}$-diphenyl(1,1'-biphenyl)-4,4'-diamine) and could accurately reproduce experimental mobilities over a range of four orders of magnitude. Even more interest was garnered by organic polymer devices, which, due to their disordered nature, can for the most part also be treated with hopping models. Examples for this are the work of Athanasopoulos et al. on polydioctylfluorene, ${ }^{237}$ the works of Nelson, ${ }^{193}$ Fornari, ${ }^{62}$ and Pershin ${ }^{238}$ and their respective co-workers on various modifications of polythiophenes, as well as the work of Walker on polyfluorenes and polyvinylenes, ${ }^{239}$ all of which rest on hopping models. In general, the straightforward applicability of the model and the wealth of relatively inexpensive methods to determine its parameters make hopping models still one of the most used models for charge transport in organic solids.

\section{Band transport regime}

The other regime in which charge transport in condensed matter is mainly discussed is the exact opposite of charge hopping. Whereas in the hopping model charges are assumed to be localized on discrete sites, band theory assumes a delocalization of charge carriers in periodic carrier bands. There is a wealth of literature on the topic of band transport, and (mainly in- 
organic) semiconductor research has made immense progress in refining band models over the years. ${ }^{240-243}$ Apart from a more complex crystal structure that is bound by a mixture of covalent and - importantly - dispersive forces, ${ }^{136}$ there is no fundamental difference between a highly pure organic and an inorganic semiconductor that would prohibit the application of these band models also to charge transport in organic solids.

The fundamental basis of band theory is Bloch's theorem. This theorem establishes the general form of an electron orbital in a periodic potential as a superposition of plane waves modified by a set of periodic functions. By construction, band theoretical approaches are thus restricted to ordered and relatively defect-free crystals, and therefore not suited for disordered semiconductors such as polymeric solids.

Considering the general definition of charge mobility of eq. (4), the defining quantity is again the field-dependent time-averaged velocity of the carrier. In this particular case, this velocity corresponds to the average of the group velocity $\mathbf{v}_{g}$ of the charge carrier's wavepacket. Given a wave-packet composed of Bloch-functions with wave-vectors near a reciprocal space vector $\mathbf{k}_{o}$ and belonging to energy band $\alpha$, the field-free group velocity is given by the reciprocal space gradient of the dispersion relation

$$
\mathbf{v}_{\mathrm{g}}=\left.\frac{1}{\hbar} \nabla_{\mathbf{k}} \varepsilon_{\alpha}(\mathbf{k})\right|_{\mathbf{k}=\mathbf{k}_{o}}
$$

Thus, all transport properties of the periodic medium are determined by its band structure represented as a reciprocal space function of electronic eigenenergies, i.e. the dispersion relation $\varepsilon_{\alpha}(\mathbf{k})$ (see Fig. 12 below for a representative example). In fact, it is relatively straightforward to show ${ }^{34}$ that the change of velocity of a particle with charge $q$ in band $\alpha$ of a periodic semiconductor and due to an applied electric field $\mathbf{E}$ simply follows a Newtonian equation of motion

$$
F_{i}=q E_{i}=\left(\frac{1}{m_{\alpha}^{*}}\right)_{i j}^{-1} \frac{d v_{\mathrm{g}, j}}{d t}
$$

albeit with a generalized effective mass $\left(\underline{\mathbf{m}}_{\alpha}^{*}\right)$, defined as the curvature of the respective band dispersion relation

$$
\left(\frac{1}{m_{\alpha}^{*}}\right)_{i j}=\frac{1}{\hbar^{2}}\left(\frac{\partial^{2} \varepsilon_{\alpha}(\mathbf{k})}{\partial k_{i} \partial k_{j}}\right) .
$$

Here, $v_{\mathrm{g}, i}$ and $E_{i}$ denote the $i$-th component of the group velocity and the electric field, respectively. Note that eq. (54) is a harmonic approximation valid only near valence band maximum (VBM) and conduction band minimum (CBM) for holes and electrons, respectively. It is therefore limited to small enough electric fields. Even then, without any dissipative terms this would imply an indefinite acceleration of charge carriers due to the electric field. Yet, in a realistic system there are other effects to consider which act in opposition to the particle's acceleration. These are mainly collisions with defects and impurities, as well as phononic lattice vibrations. The interplay between acceleration through the field and scattering at such impurities leads ultimately to a steadystate condition and thus an effective velocity for the charge carrier.

Charge transport in such a regime is statistically described by the Boltzmann transport equation, which considers the relative change in the carrier distribution functions $f$ when subjected to an external field. Thereby, it accounts for the actions of the field - driving the system out of equilibrium, the diffusion of the carriers, and collisions of the carriers with phonons and impurities, ${ }^{244}$

$$
\frac{\partial f}{\partial t}=\left(\frac{\partial f}{\partial t}\right)_{\text {field }}+\left(\frac{\partial f}{\partial t}\right)_{\text {diffusion }}+\left(\frac{\partial f}{\partial t}\right)_{\text {collision }} .
$$

It can either be solved by performing a Monte Carlo integration in momentum space ${ }^{245}$ (a technique that although successfully used for inorganic systems has not yet widely been applied to organic semiconductors) or by introducing the so called relaxation-time approximation (RTA). ${ }^{246}$ In the latter method, one linearizes the relaxation of an electron distribution in a given energy band and distorted by an external field towards the field-free ground state. 
This leads to a time constant, the relaxation time $\tau^{\mathrm{s}}$, which is the average time betweenelastic - scattering events that reset the carrier distribution in a band. In general, the relaxation time is - in exact analogy to the effective mass - a tensor of rank 2, yet for the sake of simplicity it is often transformed into the principal axes of the crystal. $^{247}$ In the following we will thus, for improved legibility, also give only the diagonal elements of the involved tensorial quantities, unless stated explicitly otherwise. For a full discussion of band-mobilities including anisotropies we refer the reader to a more specialized discussion in ref. ${ }^{248}$

In this simplified formulation and within the RTA, the carrier group velocity is given as ${ }^{249}$

$$
v_{\mathrm{g}, i}=q E_{i} \tau_{i}^{\mathrm{s}}\left(\frac{1}{m_{\alpha}^{*}}\right)_{i i} .
$$

Inserting this result for the average carrier velocity into the definition of the mobility, eq. (4), yields

$$
\mu_{i i}=q \tau_{i}^{\mathrm{s}}\left(\frac{1}{m^{*}}\right)_{i i},
$$

without any explicit dependence on the applied electric field. Additionally, we here and henceforth drop the index $\alpha$ denoting the respective band of the charge carrier. In the vast majority of cases, it is understood that the charge carrier - electron or hole-occupies its respective lowest energy configuration. As such, the bands in question are those closest to the Fermi level, the CBM for electron transport or the VBM in the case of hole conduction.

In summary, in band theory there are two parameters, two 2nd rank tensors to be precise, that need to be evaluated computationally to determine the mobility. Namely, the effective mass, which can quite straightforwardly be estimated from the material's band structure, and the relaxation time. The latter is not as straightforward to calculate. It depends on macroscopic properties such as defect concentration, long-range order and-through phononic contributions - elastic properties of the material.

\subsection{Effective Mass}

As apparent from eq. (55), the effective mass is defined as the reciprocal-space Hessian matrix of the respective energy band. Reference points for the derivatives here are the respective global extrema of the bands in the first Brillouin zone, ${ }^{34}$ i.e. the VBM and CBM. If the extrema do not coincide with the $\Gamma$-point $(\mathbf{k}=(0,0,0))$, they can easily be identified in a conventional band structure plot of the energy bands along a reciprocal space path connecting the high-symmetry points of the Brillouin zone. An example for such a plot is shown in Fig. 12. Having identified the reference point,

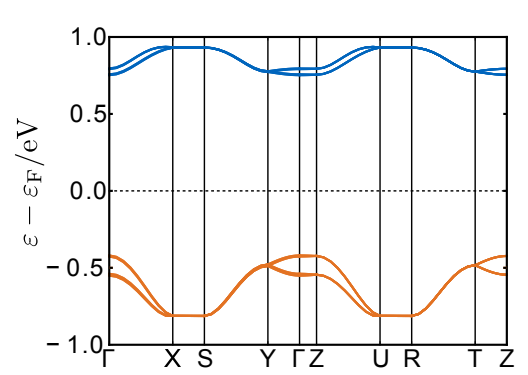

Figure 12: Semi-local (PBE) DFT valence (orange) and conduction (blue) band structure of a rubrene crystal (cf. eg. Szulczewski et al. ${ }^{250}$ ). Note the relatively large dispersion (curvature) of both, conduction and valence bands. In this case the effective mass would be estimated either at the $\Gamma$-point or the $\mathrm{Z}$ high-symmetry point. Also, given the band gap of $\approx 1.2 \mathrm{eV}$ and the Fermi level position centrally within the gap, the intrinsic charge carrier concentration would be very low (cf. section 2.1). To still yield non-vanishing conductivity, mobile charge carriers would have to be introduced either via defects or doping.

the next step is to estimate the Hessian matrix. As the band structure is generally not known in a closed analytic form, the derivatives have to be taken numerically along the three reciprocal basis vectors. Unfortunately, in three dimensions this a tedious task due to the mixed derivatives $i \neq j$.

In an isotropic medium or in the case of only one direction being of interest, there is fortunately a simpler way to estimate the effective mass. Observing that a (nearly) free electron 
gas model shows a parabolic dependence of the band energy on the reciprocal space vector in the vicinity of the band extremum with energy $\varepsilon_{0},{ }^{34}$ the following relationship holds

$$
\varepsilon(\mathbf{k})=\varepsilon_{0}+\frac{\hbar^{2}|\mathbf{k}|^{2}}{2 m^{*}},
$$

or in the fully anisotropic case

$$
\varepsilon(\mathbf{k})=\varepsilon_{0}+\frac{1}{2} \sum_{i} \sum_{j} \hbar^{2} k_{i} k_{j}\left(\frac{1}{m^{*}}\right)_{i j}
$$

with the sums running over the three components of the vector $\mathbf{k}$. In this case the effective mass $m^{*}$ can simply be acquired from a parabolic fit to energy eigenvalues calculated for a number of $\mathbf{k}$-points around the extremum.

\subsubsection{Tight-binding model of electronic band structures}

There are a number of electronic structure methods which yield the band structure for a periodic organic solid with varying degrees of accuracy and computational cost. In the semiconductor community, the prevalent method is undoubtedly DFT, but there have also been successful applications of DFTB and semiempirical methods. A straightforward way to determine the effective mass in all these methods proceeds through the explicit calculation of $\varepsilon(\mathbf{k})$ at numerous $\mathbf{k}$-points for an extended crystal described in a periodic supercell, and subsequent calculation of numerical derivatives or parabolic fitting. A different and computationally much cheaper approach for determining the band structure of organic solids was already suggested in the 1960 s by LeBlanc $^{241}$ and Jortner and co-workers, ${ }^{251}$ but is still actively developed and used today. ${ }^{252-254}$ Observing the abundance, efficiency and relative accuracy of methods to determine couplings between charge-localized states of molecular dimers and their respective on-site energies (cf. Section 3.7), they devised a tight-binding model on the basis of these states. For a crystal with two molecules sitting at two different site types 1 and 2 per unit-cell the band dispersions can then for instance be written as ${ }^{252,254}$

$$
\begin{aligned}
\varepsilon(\mathbf{k})= & \frac{\tilde{H}_{11}(\mathbf{k})+\tilde{H}_{22}(\mathbf{k})}{2} \\
& \pm \sqrt{\frac{\left(\tilde{H}_{11}(\mathbf{k})-\tilde{H}_{22}(\mathbf{k})^{2}\right.}{2}+\left(\tilde{H}_{12}(\mathbf{k})\right)^{2}}
\end{aligned}
$$

where the "+" sign holds for electrons and the "_" sign for holes. The coupling functions $\tilde{H}_{n m}(n, m=1,2)$ are related to the couplings between the molecular sites in the crystal. If analogously to reference ${ }^{254}$ — we denote the couplings between symmetrically equivalent sites in the crystal as $H_{s 1}$ and $H_{s 2}$ for site type 1 and 2, respectively, and we denote the couplings between a central molecule and its four nearest other type neighbors as $H_{n 1}, H_{n 2}, H_{n 3}$, and $H_{n 4}(n=1,2)$, respectively (cf. Fig. 13$)$, the respective periodic couplings $\tilde{H}_{n m}$ in a periodic tight-binding ansatz are given by

$$
\begin{aligned}
\tilde{H}_{11} & =2 H_{s 1} \cos \mathbf{k} \cdot \mathbf{R}_{s 1} \\
\tilde{H}_{22} & =2 H_{s 2} \cos \mathbf{k} \cdot \mathbf{R}_{s 2} \\
\tilde{H}_{12} & =H_{n 1} e^{i \mathbf{k} \cdot \mathbf{R}_{n 1}}+H_{n 2} e^{i \mathbf{k} \cdot \mathbf{R}_{n 2}} \\
& +H_{n 3} e^{i \mathbf{k} \cdot \mathbf{R}_{n 3}}+H_{n 4} e^{i \mathbf{k} \cdot \mathbf{R}_{n 4}} .
\end{aligned}
$$

As depicted in Fig. 13, $\mathbf{R}_{x}$ are thereby the direction vectors corresponding to the respective couplings listed above.

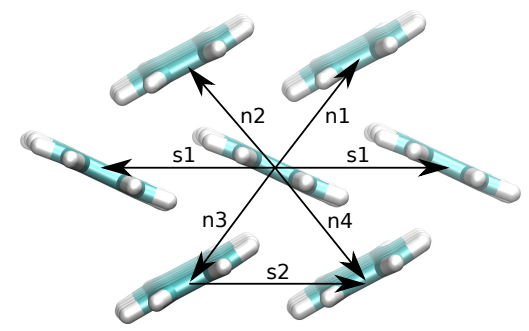

Figure 13: Charge transfer-relevant directions in a pentacene crystal. Couplings and thus band dispersion in the out-of-plane direction not pictured here are negligible.

The great advantage of such a model is obviously its closed analytical expression for the band structure. This allows for a direct analytical analysis with respect to extrema andmore importantly - an anaytical calculation of the Hessian matrix and thus the effective mass. 
The approximations underlying this approach are somewhat similar to those of the hopping model, in that only the first neighbor shell of a molecule is included, as opposed to the full periodic structure present in a crystal.

\subsection{Relaxation Time}

In order to determine the relaxation time within the RTA one has to consider the exact scattering mechanisms at play. As mentioned earlier these are mainly elastic collisions of charge carrier wave-packets with impurities, as well as with optical and acoustic phonons. If these three mechanisms are statistically independent, the total scattering rate in a direction $i$ - the inverse of the relaxation time tensor element $\tau_{i i}^{\mathrm{s}}$ - is simply the sum over the rates of all three events

$$
\frac{1}{\tau_{i i}^{\mathrm{s}}}=\frac{1}{\tau_{i i}^{\mathrm{imp}}}+\frac{1}{\tau_{i i}^{\mathrm{opt}}}+\frac{1}{\tau_{i i}^{\mathrm{ac}}}
$$

where $\underline{\tau}^{\text {imp }}$ is the relaxation time tensor for impurity scattering, and $\underline{\tau}^{\text {opt }}$ and $\underline{\boldsymbol{\tau}}^{\text {ac }}$ are the relaxation time tensors for optical and acoustic phonon scattering, respectively. All three of these can in general be determined based on Fermi's golden rule. ${ }^{249}$ Considering a scattering event that relaxes a charge carrier from state $|\alpha, \mathbf{k}\rangle$, where $\alpha$ again enumerates the energy bands and $\mathbf{k}$ is the wave-vector, to any other state $\left|\alpha^{\prime}, \mathbf{k}^{\prime}\right\rangle$ the rate is ${ }^{249,255}$

$$
\begin{aligned}
\frac{1}{\tau_{i i}} & =\frac{2 \pi}{\hbar} \sum_{\alpha^{\prime}, \mathbf{k}^{\prime}}\left|\left\langle\alpha, \mathbf{k}|\Delta \hat{V}| \alpha^{\prime}, \mathbf{k}^{\prime}\right\rangle\right|^{2} \delta\left[\varepsilon_{\alpha}(\mathbf{k})-\varepsilon_{\alpha^{\prime}}\left(\mathbf{k}^{\prime}\right)\right] \\
& \times\left(1-\frac{v_{i}(\alpha, \mathbf{k})}{v_{i}\left(\alpha^{\prime}, \mathbf{k}^{\prime}\right)}\right)
\end{aligned}
$$

where the last term is a weight function for the scattering angle. ${ }^{256}$ The operator $\Delta \hat{V}$ appearing in the coupling element between initial and final states describes the interaction of the electron with the lattice according to the given scattering mechanism.

In the case of lattice impurities, which mainly act as traps for electrons or holes, $\Delta \hat{V}$ would for example be a screened Coulomb operator. To date there are only few approaches to accurately determine $\underline{\tau}^{\text {imp }}$, even less so in the case of molecular organic solids. On the other hand, organic crystals displaying high carrier mobilities tend to be relatively pure and defect free. This results in low impurity scattering rates (large elements of $\underline{\boldsymbol{\tau}}_{\text {imp }}$ ) as compared to phononic mechanisms. The latter tend to be even more pronounced in organic semiconductors due to their weak binding and thus large thermal fluctuations. ${ }^{257,258}$ Most theoretical studies therefore focus on electron-phonon scattering as the main mechanism of charge carrier energy dissipation. ${ }^{249,254,259}$ For such charge-carrier/phonon scattering, $\Delta \hat{V}$ becomes the change of the single electron potential due to a strain on the lattice. In this case, the central matrix element in eq. (63) can for example be determined via the deformation potential model. $\Delta \hat{V}$ is there taken to depend linearly on strain with a coupling constant $\underline{\mathbf{D}}_{\text {def }}$, the eponymous deformation potential. Most generally, the latter is of course also a tensor of rank 2 , but in considering only the diagonal elements of the scattering time tensor we only need to consider diagonal elements $D_{\text {def }, i i}$ of the deformation potential. Bardeen and Shockley ${ }^{255}$ already showed in 1950 that the relaxation rate along a direction $i$ due to acoustic phonons is given by

$$
\tau_{i i}^{3 \mathrm{D}^{\mathrm{ac}}}=\frac{\sqrt{8 \pi} C_{i}^{3 \mathrm{D}} \hbar^{4}}{3\left(k_{\mathrm{B}} T\right)^{3 / 2} D_{\mathrm{def}, i i}^{2}\left(m_{i i}^{*}\right)^{3 / 2}}
$$

The deformation potential constant in direction $i, D_{\text {def }, i i}$ can in this model straightforwardly be calculated from the response of e.g. the energy eigenvalue of the band in question (VBM or $\mathrm{CBM}$ ) at the extremal $\mathbf{k}$-point $\left(\mathbf{k}_{e}\right)$ to a lattice deformation $\delta l_{i}$ along direction $i$,

$$
D_{\mathrm{def}, i i}=\left.\frac{\partial \varepsilon(\mathbf{k})}{\partial \delta l_{i}}\right|_{\mathbf{k}=\mathbf{k}_{e}} .
$$

Finally, in contrast to $D_{\text {def, } i i}$ which is the single electron response to a lattice deformation, $C_{i}^{3 \mathrm{D}}$ in eq. (64a), is the crystal's elastic constant in direction $i$ and therefore a measure for the total energetic response to lattice strain. In a harmonic picture of lattice interactions it is, 
in a 3D lattice, given by

$$
C_{i}^{3 \mathrm{D}}=\frac{1}{V} \frac{\partial^{2} E_{\mathrm{tot}}}{\partial \delta l_{i}^{2}}
$$

where $V$ is the total volume of the crystal unitcell in the unstrained configuration, and $E_{\text {tot }}$ is the total (nuclear and electronic) energy of the unit-cell.

From a computational viewpoint both $\mathbf{C}^{3 \mathrm{D}}$ and $\underline{\mathbf{D}}_{\text {def }}$ are accessible by numerical differentiation or parabolic and linear fitting procedures, respectively. This also demonstrates the simplification brought about by a treatment of band transfer in the principal axes of the crystal. Both parameters necessary to assess the relaxation time can be determined from a number of DFT geometry-optimization calculations in stretched and compressed unit-cells. ${ }^{249}$

With the above equations, it is in total quite straightforward to determine the (RTA) band mobility along the principal axes of a threedimensional periodic solid. Yet, especially in organic crystals, ${ }^{254}$ transport is often constricted to a lower dimensionality, such as 1D in the case of tightly stacked molecules, or 2D for molecules that couple only in-plane. ${ }^{254,259} \mathrm{In}$ such cases slightly modified expressions apply. The one-dimensional expression was derived by Beleznay and co-workers ${ }^{260}$ and is very similar to the 3D case. Denoting for consistency with the multi-dimensional cases the direction along which the transport occurs with the index $i$

$$
\tau_{i}^{1 \mathrm{D}}=\frac{C_{i}^{1 \mathrm{D}} \hbar^{2} \sqrt{m_{i}^{*}}}{\sqrt{2 \pi k_{\mathrm{B}} T} D_{\mathrm{def}, i i}^{2}}
$$

with the elastic constant

$$
C_{i}^{1 \mathrm{D}}=\frac{1}{l_{i}} \frac{\partial^{2} E_{\mathrm{tot}}}{\partial \delta l_{i}^{2}} .
$$

Here, the prefactor is determined by the length $l_{i}$ of the respective unit-cell vector. In the twodimensional case, the relaxation time is generally a $(2 \times 2)$ tensor, yet again we restrict the discussion to the principal axes denoted by $i$. It is given by ${ }^{261-264}$

$$
\tau_{i}^{2 \mathrm{D}}=\frac{\hbar^{3} \rho_{\mathrm{m}} v_{s}^{2} L_{\mathrm{eff}}}{k_{\mathrm{B}} T D_{i}^{2} m_{i}^{*}}
$$

where $\rho_{\mathrm{m}}$ denotes the mass density of the $2 \mathrm{D}$ system and $v_{s}$ the velocity of sound in the system. Differences in this $2 \mathrm{D}$ expression to the $1 \mathrm{D}$ and $3 \mathrm{D}$ case stem from the fact that here the relaxation expression explicitly includes the degree of localization in the direction perpendicular to the plane of transport. This is owed to the fact that restriction of the wavefunction to the $2 \mathrm{D}$ symmetry leads to quantization in only one direction, but a continuum of Bloch states in the perpendicular direction. ${ }^{261}$ This is expressed through the localization length $L_{\text {eff }}$

$$
\left(L_{\text {eff }}\right)^{-1}=\int d r_{\perp}\left|\xi\left(r_{\perp}\right)\right|^{4}
$$

where $\xi\left(r_{\perp}\right)$ is an envelope function which describes the localization along the perpendicular direction $r_{\perp}$. In the case of organic molecular crystals, it can often be approximated through the extent of the molecule in this perpendicular direction. ${ }^{254,259}$ Apart from these small differences in the underlying equations, the computational approach to determine the relaxation times in $1 \mathrm{D}$ and $2 \mathrm{D}$ is identical to that in $3 \mathrm{D}$.

As final remark, note that all of the above relations still rely on a harmonic picture of lattice vibrations, which in weakly bound organic solids might not necessarily be accurate. Improvements over the calculation of purely harmonic phonon modes can be made based on e.g. the quasi-harmonic approximation by Togo and co-workers, ${ }^{265}$ where elastic properties and phonon frequencies of a solid also show a direct dependence on the unit cell strain. While not yet applied to organic solids, such an approach should be considered for future studies of band transport in organic solids.

\subsection{Applications}

As discussed above, the coherent band-like transport regime mostly applies for highly pure and ordered organic solids. While the theoretical foundations have been proposed al- 
ready in the $1960 \mathrm{~s}$, it is only in the last 15 years that band models have increasingly been used to describe the transport in such materials. Cheng and co-workers ${ }^{252}$ determined the mobility in oligoacene single crystals based on semi-empirical estimates of the band structure. Their model first explained experimental trends of hole and electron mobilities with regards to conjugation lengths. Similarly, Giuggioli, Andersen and Kenkre ${ }^{266}$ rationalized the dependence of the mobility on temperature in pentacene. Northrup ${ }^{259}$ employed the band model in the deformation potential approximation to address the superior hole transport properties in two thiophene modifications (DNTT$\mathrm{C}_{10}$ and BTBT-C $\mathrm{C}_{12}$ ) and thin-film pentacene. Later Xi et al. ${ }^{249}$ employed an analogous approach to predict mobilities of a variety of materials ranging from graphene nano-ribbons to oligoacenes, diacene-fused thienothiophenes and various modifications thereof.

In a comparative study Kobayashi and coworkers $^{254}$ determined hopping and band mobilities for pentacene, rubrene and a benzothiophene $\left(\mathrm{C}_{8}\right.$-BTBT). In line with the theoretical considerations concerning the validity of band and hopping models (see below), as well as with experimental results, they found band transport to be the dominant mechanism at room temperature. Li et al. ${ }^{267}$ determined the temperature dependence of the effective mass in rubrene from a tight binding approach and successfully reproduced the experimentally observed conductivity behavior. Finally, very recent results revealed band-like behavior even for weakly coupled directions in highly anisotropic rubrene crystals. In a combined experimental/theoretical study, Bluelle and co-workers ${ }^{243}$ demonstrated a band-like temperature dependence of the mobility in rubrene for directions perpendicular to the strongly coupled high mobility plane. This was explained as an effect of dynamic disorder, which demonstrates again the need for a careful selection of the correct transport model for a given system.

\section{Intermediate regime}

There are numerous cases of charge transport in organic solids which do not adhere to a mechanism covered by either of the two hitherto discussed, limiting regimes. The charge carrier is neither localized at a given site, nor fully delocalized in a carrier band. With respect to electronic and electron-phonon coupling this can be seen as an intermediate regime. Before going into more detail on simulation techniques for corresponding intermediate charge transport mechanisms, we briefly summarize the ranges of validity for both limiting cases to point out why neither can yield a fully satisfactory description of charge transport in organic solids.

\subsection{Ranges of validity}

\subsubsection{Polaronic hopping model}

In order for a polaronic model to apply, the charge carrier has to exist in a localized form on one or a given (small) number of sites. In organic solids the most natural choice of such sites appears to be individual molecules in the crystal. Leaving the effect of molecular lattice vibrations aside for the moment, a localized state according to the rate theories described by eq. (13) exists as long as a barrier $\Delta G^{\dagger}>0$ separates initial and final state. In a perfect periodic crystal without any driving force the existence of such a barrier rests on the interplay between the electronic coupling and the reorganization energy, cf. Fig. 4. The crossing point from positive to vanishing activation energy can for this case be established from eq. (16), by determining the root of $\Delta G^{\ddagger}$ with respect to $\lambda$ and $H_{a b}$. This yields

$$
H_{a b} \leq \frac{1}{2} \lambda\left(\text { for } \Delta G^{0}=0\right)
$$

as a limit for a non-vanishing activation energy, and thus the existence of a small polaron. To put this relation into perspective (see also Fig. 14), in molecular crystals the reorganization free energy tends to be rather small; typically around $130 \mathrm{meV}$ in the aforementioned ex- 
ample of fullerene crystals. Equation 67 then implies that a finite barrier exists only for an electronic coupling $\lesssim 65 \mathrm{meV}$.

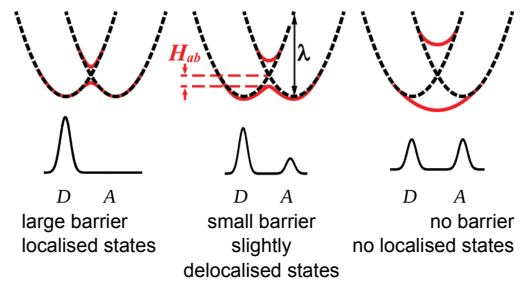

Figure 14: Illustration of the interplay of reorganization energy and electronic coupling in a two-state charge transfer model. Adapted with permission from ref. ${ }^{38}$ Copyright 2012 American Chemical Society.

Problems arise especially in organic solids with good transport properties, as these are precisely geared towards large coupling and small reorganization energies. ${ }^{38,268}$ Carrying out computations on a large database of diverse organic crystals, Schober et al. ${ }^{16}$ have shown that in many compounds electronic coupling values are above the $\lambda / 2$ threshold. This situation is further complicated when considering lattice vibrations and nuclear quantum effects. Indeed, often already the zero point motion of a large molecule along the charge transfer coordinate may hold sufficient energy to "push" it over small activation barriers. For instance, for the prominent case of $\mathrm{C}_{60}$, the corresponding $\mathrm{C}=\mathrm{C}$ stretch of the carbon cage amounts to $\approx 90 \mathrm{meV}$ of zero point energy.

In addition to the existence of a barrier, there is also a dynamic criterion for the validity of a hopping model. Ultimately based on transition state theory hopping models implicitly assume that after a hopping event, the nuclear degrees of freedom of the system return to an equilibrium in the new charge state. Yet, for a series of concerted hops, such an equilibration can only occur if the hopping rate is significantly lower than the rate of vibrational relaxation. This realization allowed Troisi ${ }^{269}$ to formulate a criterion on the maximally allowed hopping mobility to still consider a hopping mechanism. To allow a rapid evaluation, the criterion was expressed solely in materials' properties easily accessible to experiment. For a carrier of charge $e$ in a crystal with a center of mass distance between molecules $d_{a b}$, it reads

$$
\mu_{\text {hop }}<\frac{2 \pi c \tilde{\delta} q d_{a b}}{k_{\mathrm{B}} T}=\mu_{\mathrm{hop}}^{\max } .
$$

Here, $c$ denotes the speed of light, $k_{\mathrm{B}} T$ the thermal energy, and $\tilde{\delta}$ the Raman line broadening as a measure of the vibrational relaxation. Of the two system-dependent parameters, $\tilde{\delta}$ does not change much between different organic crystals and was taken as $\tilde{\delta}=$ $3 \mathrm{~cm}^{-1}$. The site-to-site distance along the maximally conducting axis of a semiconductor $d_{a b}$, on the other hand, does change significantly between crystals. In order to gauge the impact this has on the maximally allowed mobility we determined the limiting $\mu_{\mathrm{hop}}^{\max }$ for the largest $\left(d_{a b}=15 \AA\right)$ and smallest values $\left(d_{a b}=4 \AA\right)$ of $d_{a b}$ found in a recent screening study ${ }^{16}$ of organic semiconductors in the Cambridge structural database. ${ }^{15}$ This rough estimate yields $\mu_{\mathrm{hop}}^{\max }=0.03 \mathrm{~cm}^{2} \mathrm{~V}^{-1} \mathrm{~s}^{-1}(4 \AA)$ and $0.5 \mathrm{~cm}^{2} \mathrm{~V}^{-1} \mathrm{~s}^{-1}(15 \AA)$. The mobility regime where the hopping model is applicable according to Troisi's vibrational criterion is shown as an orange bar in Fig. 15.

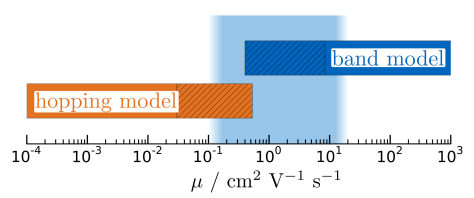

Figure 15: Illustration of the ranges of validity of hopping models (orange) and band models (blue). Ranges of the validity criterion depending on intrinsic materials' properties are depicted as hatched areas of the respective bars. The bright blue box in the background depicts the range of charge mobilities of common materials with data taken from. ${ }^{270}$ Parameters and validity criteria are described in the text, Sec. 5.1.1 for hopping and Sec. 5.1.2 for band transport, respectively.

\subsubsection{Band theory model}

The validity of the band model is closely tied to the existence of delocalized Bloch-like states in 
the crystal. Next to a near defect-free crystal structure, this implies that the mean free path of a charge carrier is much larger than the typical inter-molecular lattice spacing. ${ }^{252,271}$ Experimental relaxation times indicate that this is the case for highly pure crystals of $\mathrm{C}_{60}$, pentacene or rubrene at low temperatures. At higher temperatures though, the relaxation time and with it the mean free path length decreases significantly, indicating the formation of a more localized carrier wavefunction and therewith a breakdown of band-like transport. In analogy to the maximal mobility criterion of hopping transport, one can formulate a minimal mobility criterion observing that the mean free path length $\left\langle l_{\text {scatter }}\right\rangle$ of a carrier in one dimension is simply the product of group velocity $v_{g}$ and relaxation time $\tau_{s}$

$$
v_{g} \tau_{s}=\left\langle l_{\text {scatter }}\right\rangle
$$

Inserting this, together with the condition $\left\langle l_{\text {scatter }}\right\rangle>d_{a b}$, into the definition of the mobility, eq. (4), leads to the following criterion on $\mu$ :

$$
\begin{aligned}
& \mu_{\text {band }}>\frac{d_{a b}}{\tau_{s} E} \\
& \mu_{\text {band }}>\frac{d_{a b} q}{v_{g} m^{*}} .
\end{aligned}
$$

Here, $E$ denotes the external applied field. As this is not a property of the material, we also provide an equivalent form in eq. (70b) as a function of the effective mass $m^{*}$ and the group velocity. Compared to the hopping criterion, eq. (70b) depends on more system specific parameters, which are not necessarily easily accessible by experiment. To nevertheless provide a rough estimate for the ranges of validity we used parameters provided by Kobayashi and co-workers ${ }^{254}$ for three known band conducting materials, highly pure pentacene, rubrene, and 2,7-dioctyl[1] benzothieno[3,2b][1]benzothiophene, commonly known as C8BTBT. With group verlocities ranging from $5 \times 10^{3} \mathrm{~cm} / \mathrm{s}$ to $25 \times 10^{3} \mathrm{~cm} / \mathrm{s}$ and effective masses of $0.6 \times m_{e}$ to $1.3 \times m_{e}$ we arrive for the aforementioned representative distances of $d_{a b}=4-15 \AA$ at a range of minimally allowed mobilities of $0.4 \mathrm{~cm}^{2} \mathrm{~V}^{-1} \mathrm{~s}^{-1}$ to $8 \mathrm{~cm}^{2} \mathrm{~V}^{-1} \mathrm{~s}^{-1}$. The resulting ranges of validity are depicted in blue in Fig. 15.

Note that, while this is only a rough general estimate, each system can be gauged according to the criteria provided in eqs. (68) and (70b). Also, ranges depicted in Fig. 15 seem to suggest an overlap of validities of hopping and band transport models. This is in fact not necessarily the case, considering that due to influence of $d_{a b}$, no system can be at the same time in the ranges of high hopping mobilities but low band mobilities. Note that there exists a similar criterion for disordered solids originally provided by Mott and Davis, ${ }^{272}$ which yields similar minimal band mobilities in the range of around a few $10 \mathrm{~s}$ of $\mathrm{cm}^{2} \mathrm{~V}^{-1} \mathrm{~s}^{-1} \cdot{ }^{273}$

In addition, it is important to note that approximations to the relaxation time rest almost exclusively on a phononic description of thermal effects, cf. Section 4.2. Yet, by definition phonons are based on a harmonic view of lattice vibrations, as originally developed for inorganic solids. In organic solids, on the other hand, site-site interactions are often comparably weak (arising from dispersion forces) and internal vibrational degrees of freedom may couple to inter-molecular motion. The overall effect of both is to yield particularly pronounced anharmonic contributions to thermal molecular motion in an organic solid, which cannot be captured in a simple phononic band model. ${ }^{61,274}$

Finally, although electronic band theory does include interactions of electrons and nuclei (mostly via effective models such as the deformation potential approach), contributions due to the polarization of the surroundings are generally neglected within the assumption of full carrier delocalization. In an intermediate regime between band and small polaron model, carriers are instead still delocalized over many molecular units, but nevertheless induce a polarization response by the crystal in the form of a large polaron (cf. Fig. 16). The existence of such a spread out state does depend on temperature and polarizability of the material, and can no longer be described within the confines of traditional band theory. Effective correction 
approaches, developed to capture such effects, will be discussed in Section 5.2 below. After that, methods explicitly following the timepropagation of the charge carrier wavefunction, thus yielding an atomistic treatment without any assumptions on the mechanism, will be treated in Section 5.3.

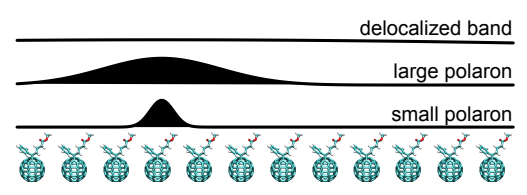

Figure 16: Illustration of charge carrier localization in a molecular crystal. (Top) Carrier probability function $|\psi|^{2}$ in a fully delocalized band (Bloch) state; (middle) large polaron delocalized over a number of molecular sites; (bottom) small polaron more or less localized on a single site. Normalization of wavefunctions here is arbitrary for illustration purposes.

\subsection{Polaronic band theories}

Early approaches to reconcile the effects of nuclear and electronic motion in band theories have been around since the late 1950s in the form of model Hamiltonians, cf. Section 4.1.1. In his pioneering work, Holstein ${ }^{122,123}$ formulated a Hamiltonian that couples electronic states locally to the phononic modes of the system. This basic approach has found many applications in the past ${ }^{275-278}$ and is also undergoing continuous improvement, the most notable being its combination ${ }^{61,279}$ with Peierls type, non-local coupling terms. ${ }^{60,280,281}$ The general form of such a Holstein-Peierls Hamiltonian for tightly bound electrons reads ${ }^{61}$

$$
\begin{aligned}
\hat{H}^{\mathrm{HP}}= & \sum_{m n} H_{m n} \hat{a}_{m}^{\dagger} \hat{a}_{n}+\sum_{\mathbf{Q}} \hbar \omega_{\mathbf{Q}}\left(\hat{b}_{\mathbf{Q}}^{\dagger} \hat{b}_{\mathbf{Q}}+\frac{1}{2}\right) \\
& +\sum_{m n \mathbf{Q}} \hbar \omega_{\mathbf{Q}} g_{\mathbf{Q} m n}\left(\hat{b}_{\mathbf{Q}}^{\dagger}+\hat{b}_{-\mathbf{Q}}\right) \hat{a}_{m}^{\dagger} \hat{a}_{n}
\end{aligned}
$$

The three terms above are, in order, the electronic term, the phononic term and the electron-phonon coupling. Thereby, $\hat{a}_{m}^{(\dagger)}$ and $\hat{b}_{\mathbf{Q}}^{(\dagger)}$ are the electronic and phononic annihila- tion (creation) operators, respectively. Sums $m, n$ run over all electronic states which are coupled by $H_{m n}$, while $\mathbf{Q}$ enumerates phonons of energy $\hbar \omega_{\mathbf{Q}}$ and their wave-vectors. $g_{\mathbf{Q} m n}$, finally, couples electron and phonon states. A coupling $\mathbf{g}$ that is diagonal in $m$ and $n$, corresponds thereby to a local (Holstein) coupling, while a purely non-diagonal $g$ leads to a fully non-local (Peierls) model.

Correctly parametrized, such a Hamiltonian leads to eigenstates that fully describe a mixed, electronic/nuclear wavefunction. ${ }^{282}$ In the past, the parametrization has been successfully achieved by fitting to experimental or theoretical data. ${ }^{61,274,277}$ Yet, in the form given above the Hamiltonian does not lend itself to a straightforward $a b$ initio parametrization or even direct diagonalization. ${ }^{61}$ It is therefore generally transformed into a more suitable basis via the so-called polaron transformation, ${ }^{61,123,283,284}$ which is unitary and thus norm-conserving. ${ }^{285}$ In the new basis comprised of electrons and lattice distortions, the explicit electron-phonon coupling terms disappear and the Hamiltonian reads ${ }^{284}$

$\hat{H}^{\mathrm{HPpol}}=\sum_{m n} \tilde{H}_{m n} \hat{a}_{m}^{\dagger} \hat{a}_{n}+\sum_{\mathbf{Q}} \hbar \omega_{\mathbf{Q}}\left(\hat{b}_{\mathbf{Q}}^{\dagger} \hat{b}_{\mathbf{Q}}+\frac{1}{2}\right)$,

where $\tilde{H}_{m n}$ now represents the coupling of states in the interacting electron-phonon system and as such depends on both the electronic, as well as the electron-phonon coupling constants. In fact, $\tilde{H}_{m n}$ is not simply a number, but instead still contains phonon operators, thus fully including the effect of polaron/phonon scattering. ${ }^{284}$ Eigenvalues of this Hamiltonian consequently represent the energy levels of the now interacting polaronic electron bands. Given the knowledge of these bands, all the methods outlined in Section 4 for determining the charge carrier mobility can be applied as well. In fact, Hannewald and co-workers ${ }^{61}$ could demonstrate a distinct narrowing of the bandwidth with temperature due to polaronic effects that is not accounted for in standard, $T=0$ band theories. This narrowing is due to a decrease of the effective coupling $\tilde{H}_{m n}$-and 
with it an increase of effective mass - with increasing temperature due to a less perfect overlap of molecular orbitals in thermally excited systems. ${ }^{286}$ Compared to purely electronic band theories, charge carriers in phononic bands are thus effectively heavier, which ultimately leads to a decrease in mobility.

In refs. ${ }^{61}$ and ${ }^{285}$ the authors also outline a way to parametrize eq. (72) from ground state, $a b$ initio calculations. They fit apurely electronic - tight binding model of the band structure, cf. eq. (61), to DFT results in order to obtain the electronic couplings. The electron-phonon coupling is then determined numerically by displacing molecules in the crystal lattice and calculating the change of coupling. ${ }^{287}$ As an alternative to this fitting procedure, any method outlined in Section 3.7 could serve to determine the couplings directly as demonstrated by Troisi for rubrene. ${ }^{282}$ Parametrization of the Hamiltonian was performed on the level of semi-empirical electronic structure calculations based on crystal geometries taken from force field MD. This study and earlier work by Girlando and co-workers ${ }^{288}$ underscored the importance of both Holstein (local) and Peierls (non-local) terms for the description of electron-phonon coupling.

Finally, it is important to note that the electron-phonon coupling term appearing in the Holstein-Peierls and related models, cf. eq. (71), is related to the reorganization free energy contributions of hopping rate theories. Applying the polaron transformation to eq. (71) leads to a new Hamiltonian, eq. (72), with coupling elements $\tilde{H}_{m n}$ that are modulated by an electronphonon term. It has been shown ${ }^{13,61}$ that the leading contribution to this modulating term can be written as a sum over Huang-Rhys factors $S_{u}$, which according to eq. (26) amounts to the reorganization free energy $\lambda$. Together with the electronic coupling $H_{a b}, \lambda$ thus appears as a defining contribution in both hopping, band (via the relaxation time approximation), and intermediate theories. Therefore, they are important, and computationally cheaply accessible, targets for in-silico screening approaches. ${ }^{16}$

\subsection{Propagation of the charge- carrier wavefunction}

Considering again that the carrier mobility, eq. (4), is defined as a function of the average drift velocity only and that, according to the generalized Ehrenfest theorem, the time evolution of such an average is uniquely determined by the Hamiltonian, a direct calculation of $\mu_{i j}$ from a dynamical simulation of the charge carrier is, in principle, possible. Altogether, such an approach amounts to solving the time-dependent Schrödinger equation for the combined nuclear-electronic system. The two most common propagation techniques used in this context are Ehrenfest based mean field (MF) dynamics ${ }^{289}$ and Tully's surface hopping (SH) method. ${ }^{290-292}$ It is important to note that both techniques, though semiclassical in that nuclear degrees of freedom are treated classically, are not tied to propagation with model Hamiltonians. Especially SH has been applied in combination with a wide variety of electronic structure methods, ranging from fully $a b$ initio approaches based on multi-reference configuration interaction, ${ }^{293}$ configuration interaction, ${ }^{294}$ and complete active space self-consistent field, ${ }^{293,295,296}$ via DFT (in the form of TDDFT ${ }^{297-299}$ and open-shell Kohn Sham ${ }^{300,301}$ ) to semi-empirical ${ }^{302-306}$ and tight-binding DFT ${ }^{307-310}$ approaches. In practice though, the high computational cost restricts the application of full ab initio methods presently to small (reference) systems. ${ }^{133,145}$

Note that both charge propagation methods ( $\mathrm{MF}$ and $\mathrm{SH}$ ) discussed below are of a semi-classical nature in that nuclear degrees of freedom are treated classically with typically only the charge carrier itself evaluated in a fully quantum mechanical fashion. Such mixed quantum-classical approaches have shown to be an optimal combination of good accuracy and reasonable computational cost for the study of complex interactions between charge carriers and phonons. ${ }^{311}$ 


\subsubsection{Mean field propagation}

In the MF or Ehrenfest approach the evolution of the classical nuclear degrees of freedom is governed by the gradient of the expectation value of the system's total energy. This includes the time-dependent quantum degrees of freedom, described e.g. by a Hamiltonian such as eq. (72). In effect this means that the classical degrees of freedom move on a single averaged potential energy surface (PES). For a time-dependent QM wavefunction $|\phi(t)\rangle$, which in the MF approach is constructed as a coherent superposition of excited states, ${ }^{289}$ the PES value experienced at time $t$ is thus given by $E_{\mathrm{QM}}(t)=\langle\phi(t)|\hat{H}| \phi(t)\rangle$. Note that in this notation $E_{\mathrm{QM}}(t)$ only contains terms relating to the quantum degrees of freedom and thus leaves out interactions between the classically treated nuclear degrees of freedom. The resulting contribution $E_{\text {class }}(t)$ can be obtained on the level of a classical force field ${ }^{93}$ (discussed in more detail below) or by coupling the mean field approach to an external bath in a Langevin like approach. ${ }^{311}$ Given such an averaged, mean field PES the equations of motion for the classical degrees of freedom $\mathbf{R}$ are simply Newtonian

$$
\begin{aligned}
\ddot{R}_{i}(t) & =-\frac{1}{M_{i}} \nabla_{i} E_{\mathrm{tot}}(t) \\
& =-\frac{1}{M_{i}} \nabla_{i}\left[E_{\mathrm{QM}}(t)+E_{\text {class }}(t)\right],
\end{aligned}
$$

where $\ddot{R}_{i}$ and $M_{i}$ denote acceleration and mass (in this case that of the charge carrier) along degree of freedom $i$, and $\nabla_{i}$ is the spatial derivative component along $i$.

The time evolution of the QM system - here the charge carrier wavefunction - is then given by the time-dependent Schrödinger equation

$$
|\dot{\phi}(t)\rangle=-\frac{i}{\hbar} \hat{H}|\phi(t)\rangle .
$$

Propagation of the combined system can be carried out with any suitable integration scheme for differential equations such as e.g. the well known Runge-Kutta integrator, ${ }^{311,312}$ of at least fourth order. ${ }^{313}$ For an in-depth analysis of advantages and disadvantages of different prop- agation schemes we refer the reader to the work of Castro and Marques. ${ }^{314}$ In order to yield results independent of the chosen initial conditions, the propagation is, in analogy to any other dynamical sampling scheme, generally repeated for a number of different initial conditions. ${ }^{311}$ For the thus resulting ensemble of "trajectories" of the wavefunction $\left|\phi^{(l)}(t)\right\rangle$, with the superscript $l$ enumerating the trajectories one then calculates the MSD. Assuming a carrier particle initially fully localized at the origin, Wang and Beljonne showed ${ }^{311}$ that the MSD can be determined from the width of the charge distribution. In this case the MSD along one of the system's principal axes $i$ is calculated by evaluating the expectation values of the carrier position operator $r_{i}$ and its square ${ }^{242}$

$$
\begin{aligned}
\operatorname{MSD}_{i}(t)=\frac{1}{L} \sum_{u=1}^{L} & {\left[\left\langle\phi^{(u)}(t)\left|r_{i}^{2}\right| \phi^{(u)}(t)\right\rangle\right.} \\
& \left.-\left\langle\phi^{(u)}(t)\left|r_{i}\right| \phi^{(u)}(t)\right\rangle^{2}\right],
\end{aligned}
$$

where $L$ is the total number of trajectories. On the other hand, Spencer and co-workers, ${ }^{93}$ in simulating the propagation of a charge carrier along a chain of molecules, used the MSD of the centre of charge for the calculation of the diffusion coefficient

$$
\operatorname{MSD}_{i}(t)=\frac{1}{L} \sum_{u=1}^{L}\left\langle\phi^{(u)}(t)\left|r_{i}\right| \phi^{(u)}(t)\right\rangle^{2} \quad
$$

Note that both definitions given here have drawbacks. Equation (75) yields zero for a fully localized quantum particle hopping from one site to the other because there the width does not change in time. Equation (76), on the other hand, gives zero if the quantum particle delocalizes symmetrically around the origin and the center of charge therefore does not move. The right definition of the MSD thus has to be chosen based on the problem at hand.

As already outlined in Section 2.2 above, the linear section of the MSD function along axis $i$ is directly proportional to the diagonal element $D_{i i}$ of the diffusion tensor $\underline{\mathbf{D}}$ of the charge car- 
rier, cf. eq. (9). This, in turn can directly be inserted into the Einstein-Smoluchowski equation, eq. (6), to yield the mobility.

Advantages of the MF approach over other methods, such as the SH scheme described next, are its simplicity and that it can be derived from first principles. Its validity for charge transport simulations, on the other hand, is very much in question. In a general context this has been shown by Tully already in $1998^{291}$ and in the case of charge transfer in organic molecular systems it was reinforced in 2013 by Wang and Beljonne. ${ }^{311}$ They showed that while MF does indeed yield diffusion tensor elements and thus numerical values of the transfer rates within the correct order of magnitude in the hopping regime, it fails to yield a correct temperature dependence of the carrier mobility. The authors were able to ultimately trace back this failure to an assumption implicitly contained in the MF approach. Use of just a single PES implies an infinite decoherence time of the charge carrier state, which in the localized (hopping) limit of transport mechanisms was shown not to be fulfilled at all. Despite these severe shortcomings ${ }^{315}$ the MF approach is employed to this day and, if used with care, can lead to physically relevant results. ${ }^{58,59,188,189,282,316-319}$

\subsubsection{Surface hopping approaches}

The SH algorithm, presented by Tully, ${ }^{290}$ improves on some aspects over the mean field approach. Owing to the method's great popularity, a number of reviews and various modifications of the original approach are available in literature. ${ }^{12,292,320,321}$ The main difference with respect to the MF method is that one considers an ensemble of realizations, progressing on a single PES at any time, but with occasional, stochastic transitions ("jumps") between surfaces instead of one realization of the system evolving on an averaged PES. The motivation for such a treatment is best illustrated with a two-state model as illustrated in Fig. 4 above. There, the dynamics of a carrier wave-packet is governed not just by the ground state adiabatic PES, but also the excited state PES, in case of an incomplete (non-adiabatic) separa- tion of electronic and nuclear degrees of freedom. As outlined above, such a behavior is indeed likely in organic solids and in effect leads to a non-vanishing probability for a wave-packet to branch between the PESs.

The SH scheme (partially) realizes such a non-zero branching probability through discrete jumps between the PESs. The dynamics of the carrier wave-packet is again determined by a time-dependent Schrödinger equation

$$
i \hbar|\dot{\Psi}\rangle=\left(\mathbb{H}-i \hbar \mathbb{d}^{\mathrm{ad}}\right)|\Psi\rangle
$$

Here, $\mathbb{H}$ denotes the adiabatic Hamiltonian matrix (cf. Section 3.2) representation in a given basis $\left\{\left|\varphi_{n}\right\rangle\right\}$, while $\mathbb{d}^{\text {ad }}$ is the non-adiabatic coupling matrix with elements

$$
d_{m n}^{\mathrm{ad}}=\left\langle\psi_{m} \mid \frac{d \psi_{n}}{d t}\right\rangle .
$$

Note that basis functions are time dependent due to the general, non-adiabatic nature of the approach, where the motion of charge carriers represented as the expansion $|\Psi\rangle=\sum_{n} c_{n}\left|\psi_{n}\right\rangle$ is not time-scale separated from the nuclear motion. Thus basis functions localized at the nuclear coordinates can not be assumed as constant in time during propagation of the carriers. We also point out that eq. (77) is in fact identical to eq. (74), yet projected onto the adiabatic states of the system.

Classical degrees of freedom are again treated simply on the level of Newtonian dynamics, cf. eq. (73). The jumps from one adiabatic PES $(m)$ to another $(n)$ then occur with a finite probability $g_{m n}$ depending on the molecular dynamics timestep $\Delta t$ and the charge carrier's density matrix $a_{m n}=c_{m}^{*} c_{n}$

$$
g_{m n}=-\Delta t \frac{2 \operatorname{Re}\left(a_{m n}^{*} d_{m n}^{\mathrm{ad}}\right)}{a_{m n}}
$$

This particular SH probability is known as the so called "fewest switches" SH (FSSH) algorithm, as it minimizes the number of transitions between PESs.

In this scheme, a single trajectory alone does not carry much physical significance due to the stochastic transitions in the dynamics. Aver- 
aged over an ensemble of trajectories with appropriately chosen initial conditions, the charge mobility can be estimated similarly to the mean field approach. ${ }^{322}$ Also, for a whole set of realizations of the dynamics starting from different initial conditions the charge mobility can be estimated similarly to the mean-field approach.

Whereas FSSH appears to be a viable approach to the simulation of charge transport in organics, the original method suffers from a number of well known shortcomings compared to a fully quantum mechanical treatment. ${ }^{321,323-326}$ Standard FSSH, for example, and indeed also the MF approach, do not treat nuclear quantum effects such as tunneling or zero-point motion by construction. ${ }^{290}$ Recently, though, this limitation has been lifted and nuclear quantum effects have been incorporated into the method by combining it with ring polymer MD. ${ }^{327-329}$

Additionally, the essential asymmetry between quantum and classical degrees of freedom in this approach can lead to an overly coherent electron dynamics often termed the "decoherence-problem", which can lead to spurious results. ${ }^{323,330,331}$ The latter can have an especially large impact on charge-transfer rates to the point where $\mathrm{SH}$ dynamics is not able to recover the Marcus limit if applied to the respective hopping regime. ${ }^{332}$ This is highly system specific though and studies on simple molecular donor-acceptor complexes for instance found the effect of missing decoherence on electron transfer rates to be rather small. ${ }^{86,333}$ Various methods to correct for the missing decoherence have been suggested. These are for example the instant collapse of the electronic wavefunction to the active state ${ }^{323}$ or exponential damping of the inactive electronic states as e.g. used in the coherent switching with decay of mixing approach ${ }^{331}$ or in the energy-based decoherence correction. ${ }^{320}$ Landry and Subotnik derived $^{334}$ an augmentation to the fewest switches SH (a-FSSH) scheme that accounts for the missing electronic decoherence. They demonstrated that by adding a decoherence step, based on a propagation of the first moments of the positions and the momenta of the charge carrier, one can indeed recover the correct Marcus be- havior. ${ }^{335}$ This step is indeed just a "collapse" of the wavefunction, that occurs with a probability proportional to the inverse of the so-called coherence time. ${ }^{335}$

A further problem in practical applications of the original FSSH scheme is its insufficient treatment of so called trivial and "unavoided" crossings ${ }^{336}$ between PESs. ${ }^{321}$ In the context of CT in organic solids they can occur between electronic states that have very small electronic interaction (e.g. when they are far apart). If they come close in energy due to nuclear fluctuations their interaction is very small which leads to the occurrence of a cusp on the two PESs pictured as a double cone with the crossing point as common vertex. The non-adiabatic coupling vector between the two states, being inversely proportional to the energy gap, shows a very sharp peak at the crossing and the hopping probability $g_{m n}$ approaches 1 . This means that in an exact simulation of the nuclear and electronic dynamics excited states could de-excite simply by going through the vertex. In numerical FSSH runs, however, the cusp can be easily missed due to the finite nuclear time step. In this case the system does not change state as it should. Consequently, since the state index after and before the crossing remains the same, the new electronic state is now the active state i.e. a spurious long-range charge transfer has occurred. ${ }^{242,337,338}$ An insufficient treatment of trivial crossings thus can lead to serious artefacts in the simulated dynamics.

To counter this, efficient modifications to the FSSH scheme have been developed in recent years. The first approach is to track trivial crossings along the trajectory, either by detecting unphysical discontinuities of the involved PESs, ${ }^{339}$ or by following the overlap between the involved adiabatic states at different times along the trajectory. ${ }^{337}$ If indicated, a transition between the PESs is then simply enforced. This approach can be somewhat tedious and becomes inefficient if trivial crossing appear frequently. Secondly, one can venture to eliminate the interaction between weakly coupled states altogether, by treating only subsystems of the material at hand with FSSH, in the so called flexible surface hopping scheme. ${ }^{242}$ 
Then, all adiabatic states are necessarily physically close, eliminating the possibility of spurious long-range charge transfer. Although the method was applied successfully to simulate CT along a harmonic chain model, ${ }^{242}$ it is likely to have some limitations. For instance, it may not solve the trivial crossing problem in cases where electronic couplings between physically close diabatic states are very small or when the charge carrier is spatially strongly delocalized. Recently, Prezhdo and co-workers suggested an elegant and effective detection method for trivial crossings based on a rigorous sum rule for hopping probabilities. ${ }^{338}$ Violation of the sum rule at a given MD step indicates occurence of a trivial crossing, in which case the hopping probability to the adiabatic state closest in energy to the active adiabatic state is corrected. This method was shown to give excellent results for model systems. ${ }^{338}$ The only caveat is that it may not provide a correction to the hopping probability where more than two surfaces are involved in a trivial surface crossing.

A further drawback of the FSSH approach was shown by recent analyses for small model systems. ${ }^{340,341}$ These showed that surface hopping does not generally yield the exact equilibrium Boltzmann distribution of electronic states. The deviations from the exact Boltzmann limits, however, were also found to be small especially for systems with small electronic and strong non-adiabatic couplings.

Finally, it is evident that-compared to the single PES considered in the MF approachthe sampling efficiency of surface hopping is directly determined by the number of excited state surfaces that need to be included to describe a particular system. In metallic systems, for example, ${ }^{342}$ FSSH becomes very inefficient due to the large number of PES' contributing to a single band. In this case, hops occur extremely frequently as the energetic separation between the PES is small at any time requiring a large number of trajectories for proper sampling. ${ }^{342}$ Whether or not this will become a problem for the simulation of charge transport in organics remains to be seen.

In summary, recent research provided a great number of improvements over the original
FSSH scheme each dealing with different shortcomings. For each application the combination of these techniques has to be carefully chosen based on the processes expected to occur. A more in-depth review of FSSH and its stateof-the-art improvements can be found in reference. $^{321}$

\subsubsection{Other propagation methods}

Before going on to discuss a specific example of a combined nuclear-electronic dynamics we here briefly describe other techniques, which, though not yet as widespread in the field of organic solids, can also be employed to model CT processes.

Realtime time-dependent DFT As already mentioned in the introduction to Section 5.3, neither MF nor FSSH or its derivatives are limited to specific descriptions of the electronic degrees of freedom. Although we have, for the sake of brevity, limited the discussion to dynamics based on electronic model Hamiltonians, a more versatile electronic description exists e.g. in the form of realtime time-dependent DFT (RT-TDDFT). ${ }^{343}$ This is based on the Runge-Gross theorem, ${ }^{344}$ which is the timedependent analogue of the first HohenbergKohn theorem. ${ }^{345}$ It states that there is an exact mapping between the time-dependent density $\rho(\mathbf{r}, t)$ and the time-dependent potential $V(\mathbf{r}, t)$ and thus by extension also the timedependent wavefunction. Yet, analogously to ground state DFT, not every observable can easily be expressed in terms of the density. Thus, based on the work of van Leeuwen, ${ }^{346}$ one generally employs a reference system of non-interacting, though time-dependent, single electron orbitals with the same density as the full system. This treatment leads to a Hamiltonian comparable to ground state KohnSham DFT $^{347}$ in that it includes an unknown but time-dependent exchange-correlation (XC) term that needs to be approximated. This is, however, complicated by the fact that the hypothetical "exact" term couples XC contributions at different times leading to a memory effect. ${ }^{348,349}$ A number of studies investigated 
the effect of memory on RT-TDDFT, for example using an adiabatically exact approximation to the XC potential, which completely neglects XC-memory. ${ }^{350-353}$ Unfortunately, such a simple approach was shown to fail for CT problems, ${ }^{351,353}$ making it unsuitable for the tasks discussed in this review. Yet, there is ongoing research into more accurate approximations to the time-dependent XC functionals and more in-depth discussion of RT-TDDFT can for example be found in reference. ${ }^{343}$ Finally, while RT-TDDFT provides a way to propagate electronic degrees of freedom, the coupling to nuclear motion is commonly achieved in Ehrenfest like approaches. ${ }^{289,354,355}$ Yet, more accurate, though also more expensive, multiconfigurational approaches to RT-TDDFT have been reported in the literature. ${ }^{356,357}$

\section{Multi-configurational time-dependent} SCF approaches Propagation of a charge carrier in an extended system with two or more possible states in principle needs to take into account the PES' of all states, as outlined in Sec. 5.3.2 above. Yet, while the $\mathrm{SH}$ and $\mathrm{MF}$ approaches treat these in an explicitly but stochastically or in an averaged fashion, respectively, the family of multi-configurational time-dependent SCF (MC-TDSCF) ${ }^{358}$ methods represents an approximate but deterministic treatment of time-dependent problems. The most common variant nowadays, which we very briefly discuss here, is the multi-configurational time-dependent Hartree (MCTDH) method. ${ }^{359,360}$ This is based on the expansion of the full solution to the timedependent Schrödinger equation into Hartree products of time-dependent single electron orbitals. ${ }^{361}$ Inserting this ansatz together with a constraint operator establishing the orthogonality of eigenfunctions at every time step, into the Schrödinger equation yields a set of coupled equations for both the single particle orbitals and the expansion coefficients of the full wavefunction. Given a large enough basis of product functions, MCTDH can thus yield an accurate picture of the time evolution of any prepared initial state. The advantages of MCTDH in the context of this review are thereby two-fold. First, the combined electronnuclear dynamics is fully non-adiabatic and thus in-principle not biased to one specific CT mechanism. ${ }^{362}$ The second advantage lies in its systematic improvability, where by adding more product functions one can get an increasingly accurate description. Unfortunately, with the number of product functions the computational cost of MCTDH also grows steeply, which means that early applications where confined to very small systems. ${ }^{360}$ Yet, recent developments such as multi-layer MCTDH, ${ }^{363-365}$ where the large number of single particle functions is treated in a hierarchical tree-like fashion, has reduced the cost of the method such that now thousands of degrees of freedom can be accurately treated for short timescales. First simulations of quantum dynamics of organic systems with MCTDH were reported e.g. by Burghardt and co-workers, ${ }^{366-368}$ investigating exciton dynamics and dissociation in oligothiophenes and thiophene-fullerene junctions, or by Zheng et al. ${ }^{369}$ studying singlett fission in a pentacene dimer model system. All these studies share their focus on ultra-fast processes, owing to the still present resource limitations of the method. A more detailed review of MCTDH and its applications can be found in a focused review by Beck, Jäckle, Worth and Meyer. ${ }^{361}$

\subsubsection{Example: propagation in a charge localized basis}

To illustrate how explicit time propagation can be realized in practice, we discuss an efficient FSSH scheme recently put forward by Spencer, Gajdos and Blumberger. ${ }^{93}$ Inspired by CT rate theory approaches and CDFT, they expand the time-dependent electronic wavefunction in a basis of charge localized SOMOs. This approach rests on a number of assumptions, first and foremost that the excess charge may be described by a one-particle wavefunction expressed as a linear combination of SOMO orbitals of the molecules. Interactions between carriers were not included in their first version, hence limiting its applicability to the low carrier concentration limit.

The basis functions $\left|\varphi_{m}\right\rangle$ used in the scheme 
are then analogous to the diabatic states used in rate theoretical approaches (see Section 3.1) in that each of them represents a state where the excess charge carrier is entirely localized on a given molecular site. Note that the name diabatic state does not strictly apply to such reference states, as they do not diagonalize the non-adiabatic coupling matrix. This is why Spencer and co-workers refer to them as SOMOs. An illustration of this SOMO basis for a fullerene derivative is given in Fig. 17. In order

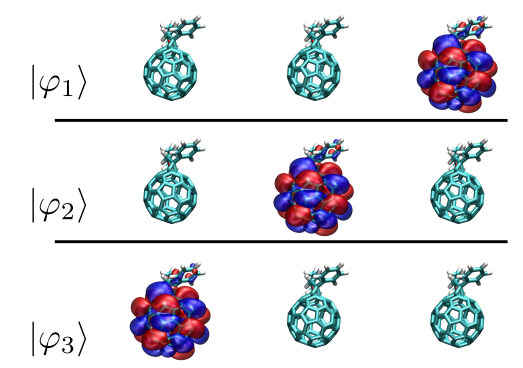

Figure 17: 1D illustration of the chargelocalized SOMO basis used in the fragment orbital based surface hopping (FOB-SH) approach for a chain of PCBM molecules. Each basis function $\left|\varphi_{n}\right\rangle$ is given as a charge carrier (electron or hole) localized on different sites in the chain.

to solve the time-dependent Schrödinger equation, eq. (77), the wavefunction $|\psi\rangle$ and thus the Hamiltonian $\mathbb{H}$ and the non-adiabatic coupling matrix $\mathbb{d}^{\text {ad }}$ are expanded in the SOMO basis for every given snapshot $\left\{\mathbf{R}_{k}(t)\right\}$ of nuclear positions along a trajectory. Comparing to diabatic rate theories, off-diagonal elements of the Hamiltonian $\left\langle\varphi_{m}|\hat{H}| \varphi_{n}\right\rangle$ are in this approach simply given by the electronic coupling elements. These elements can in principle be determined with any of the methods discussed in Section 3.7. Yet, for practical reasons such as scaling and computational cost this determination is presently restricted to cheaper methods such as FO-DFT or AOM. The diagonal elements of the Hamiltonian $\left\langle\varphi_{m}|\hat{H}| \varphi_{m}\right\rangle$, in turn, represent the site energies of such chargelocalized states within the potential of the crystal. As such, they include contributions beyond simple two-site approximations used for the offdiagonal elements. Again for reasons of compu- tational efficiency, Spencer et al. here employ a classical force field, which yields the energetics of the charge response to a given localization of the excess electron. Finally, the non-adiabatic coupling elements $d_{m n}^{\prime}(t)$ between the charge localized states are estimated by finite differences from the overlap matrices of the SOMO basis functions taken at different times

$$
\begin{aligned}
d_{m n}^{\prime}(t) & =\left\langle\varphi_{m}(t) \mid \frac{d}{d t} \varphi_{n}(t)\right\rangle \\
& \approx \frac{1}{\Delta t}\left(\left\langle\varphi_{m}(t) \mid \varphi_{n}(t+\Delta t)\right\rangle\right. \\
& \left.-\left\langle\varphi_{m}(t) \mid \varphi_{n}(t)\right\rangle\right)
\end{aligned}
$$

These are then transformed into the adiabatic basis to yield the non-adiabatic coupling elements $d_{m n}^{\mathrm{ad}}(t)$ (cf. eq. 78). Here, the analytical time-derivative of the basis function $\left|\varphi_{n}(t)\right\rangle$ has been replaced by a numerical approximation with a small but finite time step $\Delta t$, which for convenience can be chosen as the time step for the propagation of the nuclei.

The thus fully determined Hamiltonian and non-adiabatic coupling matrix can not yet be used directly for propagation within the SH scheme. For this, they have to be unitary transformed into an orthogonalized basis, for instance through Löwdin's symmetric approach. ${ }^{211-213}$ Note that the transformation of the non-adiabatic coupling thereby also necessitates calculation of the time derivative of the transformation matrix, which in the present approach is calculated analogously to $\mathbb{d}^{\prime}$. With the transformed matrices a prepared initial state is then propagated according to eq. (77) using a fourth order Runge-Kutta scheme. ${ }^{370}$ The necessary nuclear gradients were calculated by Spencer et al. in a mixture of analytical and numerical schemes, as described in detail in ref. ${ }^{93}$ Given a number of charge trajectories the carrier mobility was finally estimated by evaluating the MSD of eq. (75) and exploiting the EinsteinSmoluchowski relation, eq. (6), in close analogy to the MF approach described in Section 5.3.1.

By specifically choosing localized fragment orbitals as basis for the expansion of Hamiltonian and non-adiabatic coupling Spencer et al. exploited a number of advantages, such as 
a possible linear scaling behavior due to the sparsity of the resulting matrices, as well as easy access to the necessary matrix elements for a given nuclear configuration. On top of that, the localized approach somewhat mitigates the well known charge-delocalization errors of DFT. ${ }^{95,371}$ Yet, at least in the form presented in reference, ${ }^{93}$ there are also a number of caveats to such an approach. For example, only intra-band CT in the band formed of the SOMOs is considered by construction. Therefore, the dynamics of optical excitations ("excitons"), occurring for example in organic solar cells, could not be addressed with this approach. Moreover and as mentioned previously, no interaction between charge carriers is incorporated yet. In future work, it may be possible to model these effects by suitably expanding the state space of the electronic Hamiltonian.

\subsection{The Kubo formula}

Finally, we point out that the charge carrier mobility in a material can also be expressed in terms of a time-correlation function of the carrier current $I(t)$ based on the Kubo formula (KF). ${ }^{54,372}$ Especially considering the relative ambiguity of organic molecular crystals with respect to the carrier transport regime, an unbiased approach based on statistical considerations such as the KF can be of great importance. Indeed, a number of recent studies of organic materials based their approaches on the KF. Ortmann, Bechstedt, and Hannewald, ${ }^{284}$ for example, applied the KF to a Holstein Hamiltonian - cf. eqs. (71) and (72) - to determine carrier mobilities for a realistic model system, showing the temperature-induced transition from hopping-dominated to band-like transport. Similarly, Fratini and Ciuchi ${ }^{257}$ employed the KF to determine the carrier mobility in rubrene as a function of temperature, demonstrating the versatility of KF approaches to describe both band-like and incoherent transport mechanisms. Similarly Gunnarsson and Han, ${ }^{373}$ on the other hand explored the mean free carrier path length in gap-less and therefore metallic fullerene crystals. Wang et al. ${ }^{374}$ employed the related Kubo-Greenwood formal- ism to study the charge transport properties in different phases of polyethylene.

In the limit of vanishing momentum transfer from the carrier to the lattice the $\mathrm{KF}$ can be expressed as ${ }^{257}$

$$
\mu=\frac{\beta \pi e d^{2}}{Z \hbar} \int d \omega B(\omega) e^{-\beta \omega},
$$

with $d$ the average spacing between crystal sites and

$$
B(\omega)=\int d t e^{i \omega t}\langle[I(t), I(0)]\rangle
$$

the Fourier transform of the current autocorrelation function. It can be viewed as an energy-resolved, state-specific mobility function and can be calculated e.g. from the expectation value of the current operator $\sum_{j}\langle\psi(t)| \hat{a}_{j}^{\dagger} \hat{a}_{j-1}-$ $\hat{a}_{j}^{\dagger} \hat{a}_{j+1}|\psi(t)\rangle$, where $\hat{a}^{\dagger}, \hat{a}$ are the creation and annihilation operators and the sum runs over all states. Finally,

$$
Z=\int d \omega \rho(\omega) e^{-\beta \omega}
$$

with the density of states $\rho(\omega)$. Gunnarsson and $\operatorname{Han}^{373}$ demonstrated that the correlation function $B(\omega)$ can-by neglecting so called vertex corrections-be factorized into Green's function terms and matrix elements of the current operator, both averaged over the phonon displacement spectrum. Using this, Fratini and Ciuchi ${ }^{257}$ further simplified the calculation of $B(\omega)$ to just the calculation of the system's Green's function, observing that the latter term can be determined analytically. Moreover, Wang and co-workers ${ }^{374}$ demonstrated that using a result from KuboGreenwood theory ${ }^{372,375,376}$ one can reduce the computational complexity even further, essentially replacing the current correlation function with a mean square momentum matrix element between pairs of different states, averaged over all states.

\subsection{Applications}

Driven by an increasing awareness that neither hopping nor band models alone are able 
to fully explain experimentally reported findings, there has been a strong shift toward the more general approaches in the last decade. As already mentioned in Section 5.2 Hannewald and co-workers ${ }^{61,279}$ conducted seminal studies of polaronic bandwidth narrowing in oligoacene molecular crystals (naphtalene, anthracene, and tetracene) based on a HolsteinPeierls Hamiltonian. Troisi and Orlandi ${ }^{274,316}$ subsequently showed for the examples of pentacene and anthracene, that the thermal modulation of the electronic couplings entering such model Hamiltonians cannot be neglected.

Next to polaronic band theories especially non-adiabatic propagation of the charge carrier wavefunction has been employed to study transport in organic crystals. Troisi ${ }^{282}$ demonstrated the accuracy of such approaches versus experiments for the absolute charge mobility in rubrene. Using a mixed quantum/classical approach ${ }^{315}$ based on SH Wang and Beljonne 242,311 studied the crossover from hopping to band-like transport in organic crystals. Demonstrating the versatility of the method, Ren and co-workers ${ }^{377}$ also employed non-adiabatic MD to simulate charge transport in two-dimensional pentathiophene butyric acid monolayers to determine the underlying mechanisms of charge transport in such confined systems. Based on a quantized Hamiltonian dynamics, first Hannewald and Bobbert, ${ }^{279}$ and later again Wang et al. ${ }^{378}$ explained low-temperature experimental results for charge transport in naphtalene crystals. Zhang, Zhong, and Zhao ${ }^{379}$ employed a timedependent wavepacket method to explain electron mobilities in pentacene-quinone derivatives, also finding a crossover from hopping to band-like behavior. For chains of ethylenelike molecules Spencer et al. ${ }^{86,93}$ demonstrated that their just discussed fragment-orbital based non-adiabatic SH method achieves comparable accuracy to these earlier and computationally more expensive methods. A conceptually similar method, brought forward by Heck and coworkers, ${ }^{188,189}$ also utilizes a fragment orbital description, based on DFTB, to arrive at an analogous effective QM-MM single-particle description of the charge carrier. In contrast to the FOB-SH, Heck et al. propagated the charge carrier with Ehrenfest dynamics, which has a number of well known deficiencies (cf. section 5.3.1). Nonetheless, the charge mobility computed for chains of organic molecules was in good agreement with experimental data, where available.

Finally, dynamic approaches to charge transport are not limited to periodic crystalline materials. For example, Akimov ${ }^{194}$ utilized a fragment orbital scheme based on semi-empirical extended Hückel theory in the non-adiabatic SH propagation of an effective QM-MM Hamiltonian of a molecular donor-acceptor heterojunction of Boron Subphthalocyanine Chloride and $\mathrm{C}_{60} \cdot{ }^{380}$ Johansson and Stafström ${ }^{381,382}$ as well as An, Wu and Sun ${ }^{383}$ employed non-adiabatic dynamical approaches to study polaron transport along polymer chains. In another recent approach, Bauer et al. ${ }^{384}$ used a multi-agent quantum Monte Carlo model to model charge transport through disordered multi-phase field effect transistors consisting of $\mathrm{C}_{60}$ molecules sitting on a layer of alkyl chains.

\section{Synopsis, remaining chal- lenges and future direc- tions}

In the present review, we set out to provide a comprehensive overview over the tools currently available to the computational scientist to tackle the study of charge carrier transport in molecular organic semiconductors. To this end we first gave a summary of the theories of conductivity in Section 2, highlighting the different regimes of transport occurring in organic semiconductors. Then we elaborated them in detail in subsequent Sections. In Section 3 we first detailed the theoretical background of the hopping model, thereby pointing out the different limiting cases of hopping rate theories available in literature. This discussion included all common computational methods available to determine the parameters going into rate theories for an ab initio calculation of charge carrier mobilities within the hopping model. 
Where the hopping model assumes a localized, polaronic charge carrier, band theory rests on the opposing assumption of a delocalization of carriers in periodic bands. Corresponding approaches were described in Section 4. There we again first detailed the theoretical foundations of the model before giving an overview over computational methods available in the theorist's toolbox. Applicability of either model rests on the intricate interplay of charge transfer parameters in each semiconductor. Which model to apply is therefore not necessarily possible to decide beforehand, in some cases even the use of neither model can reasonably be justified. In such intermediate cases, dynamic simulations as described in Section 5 have to be employed. To set this regime into perspective we first discussed in detail the ranges of validity of the limiting carrier transport regimes. Available methods for the intermediate regime comprise the propagation of model Hamiltonians or the explicit propagation of the wavefunction. Finally, we ended the main part of this manuscript with a discussion of the famous Kubo transport equation in Section 5.4, which represents a statistical approach to charge transport in solids, considering timecorrelation functions of the charge current.

Given such a wealth of methods available to the theoretical researcher, one would be tempted to assume that most challenges in simulating organic semiconductors have been overcome and one can readily employ theoretical methods to predict charge transfer parameters of realistic organic solids. As we have pointed repeatedly, this is unfortunately not the case. Direct comparison of absolute mobilities to experiment, is still only rarely possible for several reasons. On the theoretical side, as pointed out in Section 5.1, the exact mechanism underlying charge carrier transport in a system is often unclear or even ill-defined. In such cases only propagation methods - discussed in Section 5 can in principle deliver an unbiased picture. Yet, even such methods rest on a number of approximations, given the large sizes of organic molecular systems. On the other hand, experimental studies have their own unique set of challenges to contend with. For instance, mo- bility measurements of one and the same system can deliver wildly differing values mostly due to minute differences in preparation leading to ill-defined or disordered crystal structures. Many theoretical studies nowadays recognize the problem and introduce models for the influence of structural disorder on electronic couplings, Sec. 3.7, and on-site energies, Sec. 3.3.1, which indeed show first successes. Similarly, dynamical influences on carrier localization and propagation, through electronphonon coupling, Sec. 4.2, and a certain degree of dynamical disorder, are gathering increased attention for more refined modelling studies.

Another challenge for the comparison of theory and experiment are interface effects. While this review concentrated mainly on techniques for the study of bulk charge transport phenomena, virtually every real-life application of such systems - and thus also experimental measurements - needs to interface the semiconducting organic solid with metal electrodes. Disregarding for the moment the problem of structural modelling of the interface, transport across such interfaces is by necessity harder to model, as one needs to combine methods suited for metallic systems with those geared towards molecular transport. In the case of single-molecule/metal interfaces a great variety of theoretical studies can be found in literature due to their potential application in molecular electronics. Yet, with regards to the theory of charge carrier propagation across bulk interfaces between organic solids and metals there is still much work do be done.

Another important aspect is the geometric structure, which necessarily underlies all theoretical studies. While this review focused on ordered organic solids, the class of amorphous or polymeric organic solids is of high interest for applications in display devices, electronics, and even solar cells. The ill-defined microscopic structure of such materials poses a significant challenge to any atomically resolved modeling. One way to obtain representative structures used for charge transport calculations is to perform MD simulations, possibly performed at elevated temperatures and based on approximate energetics to lower the computational 
burden. Snapshots drawn from the computed trajectories then provide an ensemble of structures which can then be treated using the methods outlined in this review, ultimately yielding a distribution of charge transport parameters. While amorphous semiconductors could in principle also be tackled through such snapshots, sampling the likely non-equilibrium distribution of their structures poses a practical problem. Such a discrepancy in structure distributions can thus potentially lead to large discrepancies between experiment and theory.

Finally, one of the great advantages of theory, even given all of the problems outlined in the paragraphs above, is to point out trends and thus provide a link between structure and function of a material. Given such a link, one could think of employing a number of easily computable parameters for a given system, such as coupling and reorganization energy as discussed in Sec. 5.2, to at least roughly predict its applicability as an organic semiconductor relative to other materials in a computational screening study. Yet, as before, such screening approaches again depend on the knowledge of the microscopic structure of the material, which in principle limits their applicability to already known systems. Luckily, the last couple of years saw great leaps in crystal structure prediction methodology, which brought us to the point where, for any molecule, one can predict to a great degree of accuracy the corresponding crystal structure. ${ }^{22}$ While these prediction studies tend to be costly, theoretical predictability of charge transport properties for unknown systems is now definitely within our reach.

In conclusion, then, what is the ideal method to describe CT? Unfortunately, there is no simple answer to this question, as the ideal method will always depend on the system, the regime, the available resources, and finally the exact question one wants to answer. In general, an ideal theory should yield the transport mechanism as a result and not assume one in the first place. This in turn points towards the methods discussed in Section 5. At the same time one ideally would like to be able to treat large or even disordered systems which, given even today's computing resources, can be a challenge for methods relying on direct charge propagation. On the other hand, as outlined in Sec. 5.2, if one is only interested in broad mobility trends in a screening study, in principle only few parameters have to be calculated per system. Then a full non-adiabatic dynamics clearly would go far beyond what is necessary in practice. Thus, the question at hand as well as the system and the available resources inform the choice of method to be used and with this review the authors aimed at providing a guide to the available approaches in the different regimes for the reader to choose from.

Acknowledgement H.O. and K.R. gratefully acknowledge support from the Solar Technologies Go Hybrid Initiative of the State of Bavaria. With the support of the Technische Universität München Institute for Advanced Study (TUM-IAS), funded by the German Excellence Initiative and the European Union Seventh Framework Programme under grant agreement $\mathrm{n}^{\circ} 291763$ J.B. acknowledges the European Union for co-funding of a TUM-IAS Hans-Fischer Fellowship. Finally, the authors thank Christoph Schober, Christoph Scheurer and Wolfgang Domcke for their valuable input and insightful discussions.

\section{References}

(1) Forrest, S. R. The Path to Ubiquitous and Low-Cost Organic Electronic Appliances on Plastic. Nature 2004, 428, 911918.

(2) Gundlach, D. J. Organic Electronics: Low Power, High Impact. Nat. Mater. 2007, 6, 173-174.

(3) Minemawari, H.; Yamada, T.; Matsui, H.; Tsutsumi, J.; Haas, S.; Chiba, R.; Kumai, R.; Hasegawa, T. Inkjet Printing of Single-Crystal Films. Nature 2011, 475, 364-367.

(4) Sun, Y.; Welch, G. C.; Leong, W. L.; Takacs, C. J.; Bazan, G. C.; Heeger, A. J. Solution-Processed Small-Molecule Solar 
Cells with $6.7 \%$ Efficiency. Nat. Mater. 2012, 11, 44-48.

(5) Anikeeva, P.; Koppes, R. A. Restoring the Sense of Touch. Science 2015, 350, 274-275.

(6) Stavrinidou, E.; Gabrielsson, R.; Gomez, E.; Crispin, X.; Nilsson, O.; Simon, D. T.; Berggren, M. Electronic Plants. Sci. Adv. 2015, 1, e1501136.

(7) Newton, M. D.; Sutin, N. Electron Transfer Reactions in Condensed Phases. Annu. Rev. Phys. Chem. 1984, 35, 437480 .

(8) Nitzan, A. Electron Transmission through Molecules and Molecular Interfaces. Annu. Rev. Phys. Chem. 2001, 52, 681-750.

(9) Brédas, J.-L.; Beljonne, D.; Coropceanu, V.; Cornil, J. ChargeTransfer and Energy-Transfer Processes in $\pi$-conjugated Oligomers and Polymers: a Molecular Picture. Chem. Rev. 2004, 104, 4971-5004.

(10) Coropceanu, V.; Cornil, J.; da Filho, D. A. S.; Olivier, Y.; Silbey, R.; Brédas, J.L. Charge Transport in Organic Semiconductors. Chem. Rev. 2007, 107, 926-952.

(11) Voorhis, T. V.; Kowalczyk, T.; Kaduk, B.; Wang, L.-P.; Cheng, C.L.; Wu, Q. The Diabatic Picture of Electron Transfer, Reaction Barriers, and Molecular Dynamics. Annu. Rev. Phys. Chem. 2010, 61, 149-170.

(12) Akimov, A. V.; Neukirch, A. J.; Prezhdo, O. V. Theoretical Insights into Photoinduced Charge Transfer and Catalysis at Oxide Interfaces. Chem. Rev. 2013, 113, 4496-4565.

(13) Zhugayevych, A.; Tretiak, S. Theoretical Description of Structural and Electronic Properties of Organic Photovoltaic Materials. Annu. Rev. Phys. Chem. 2015, 66, 305-330.
(14) Blumberger, J. Recent Advances in the Theory and Molecular Simulation of Biological Electron Transfer Reactions. Chem. Rev. 2015, 115, 11191-11238.

(15) Groom, C. R.; Bruno, I. J.; Lightfoot, M. P.; Ward, S. C. The Cambridge Structural Database. Acta Crystallogr. Sect. B 2016, 72, 171-179.

(16) Schober, C.; Reuter, K.; Oberhofer, H. Virtual Screening for High Carrier Mobility in Organic Semiconductors. J. Phys. Chem. Lett. 2016, 7, 3973-3977.

(17) Lommerse, J. P. M.; Motherwell, W. D. S.; Ammon, H. L.; Dunitz, J. D.; Gavezzotti, A.; Hofmann, D. W. M.; Leusen, F. J. J.; Mooij, W. T. M.; Price, S. L.; Schweizer, B. et al. A Test of Crystal Structure Prediction of Small Organic Molecules. Acta Crystallogr. Sect. $B$ 2000, 56, 697-714.

(18) Motherwell, W. D. S.; Ammon, H. L.; Dunitz, J. D.; Dzyabchenko, A.; Erk, P.; Gavezzotti, A.; Hofmann, D. W. M.; Leusen, F. J. J.; Lommerse, J. P. M.; Mooij, W. T. M. et al. Crystal Structure Prediction of Small Organic Molecules: A Second Blind Test. Acta Crystallogr. Sect. B 2002, 58, 647-661.

(19) Day, G. M.; Motherwell, W. D. S.; Ammon, H. L.; Boerrigter, S. X. M.; Della Valle, R. G.; Venuti, E.; Dzyabchenko, A.; Dunitz, J. D.; Schweizer, B.; van Eijck, B. P. et al. A Third Blind Test of Crystal Structure Prediction. Acta Crystallogr. Sect. B 2005, 61, 511-527.

(20) Day, G. M.; Cooper, T. G.; CruzCabeza, A. J.; Hejczyk, K. E.; Ammon, H. L.; Boerrigter, S. X. M.; Tan, J. S.; Della Valle, R. G.; Venuti, E.; Jose, J. et al. Significant Progress in Predicting the Crystal Structures of Small Organic Molecules - A Report on the Fourth Blind Test. Acta Crystallogr. Sect. B 2009, 65, 107-125. 
(21) Bardwell, D. A.; Adjiman, C. S.; Arnautova, Y. A.; Bartashevich, E.; Boerrigter, S. X. M.; Braun, D. E.; CruzCabeza, A. J.; Day, G. M.; Della Valle, R. G.; Desiraju, G. R. et al. Towards Crystal Structure Prediction of Complex Organic Compounds - A Report on the Fifth Blind Test. Acta Crystallogr. Sect. B 2011, 6r, 535-551.

(22) Reilly, A. M.; Cooper, R. I.; Adjiman, C. S.; Bhattacharya, S.; Boese, A. D.; Brandenburg, J. G.; Bygrave, P. J.; Bylsma, R.; Campbell, J. E.; Car, R. et al. Report on the Sixth Blind Test of Organic Crystal Structure Prediction Methods. Acta Crystallogr. Sect. B 2016, 72, 439-459.

(23) Wales, D. J.; Scheraga, H. A. Global Optimization of Clusters, Crystals, and Biomolecules. Science 1999, 285, 13681372 .

(24) Wales, D. Energy Landscapes: Applications to Clusters, Biomolecules and Glasses; Cambridge University Press, 2003.

(25) Kwiatkowski, J. J.; Frost, J. M.; Nelson, J. The Effect of Morphology on Electron Field-Effect Mobility in Disordered C60 Thin Films. Nano lett. 2009, 9, 1085-1090.

(26) MacKenzie, R. C.; Frost, J. M.; Nelson, J. A Numerical Study of Mobility in Thin Films of Fullerene Derivatives. J. Chem. Phys. 2010, 132, 064904.

(27) Rühle, V.; Lukyanov, A.; May, F.; Schrader, M.; Vehoff, T.; Kirkpatrick, J.; Baumeier, B.; Andrienko, D. Microscopic Simulations of Charge Transport in Disordered Organic Semiconductors. J. Chem. Theory Comput. 2011, 7, 3335-3345.

(28) Baumeier, B.; May, F.; Lennartz, C.; Andrienko, D. Challenges for In Silico Design of Organic Semiconductors. J. Mater. Chem. 2012, 22, 10971-10976.
(29) Silinsh, E. A. Organic Molecular Crystals: their Electronic States; Springer Science \& Business Media, 2012; Vol. 16.

(30) Nazin, G.; Qiu, X.; Ho, W. Vibrational Spectroscopy of Individual Doping Centers in a Monolayer Organic Crystal. J. Chem. Phys. 2005, 122, 181105-181105.

(31) Giovannetti, G.; Brocks, G.; van den Brink, J. Ab Initio Electronic Structure and Correlations in Pristine and Potassium-Doped Molecular Crystals of Copper Phthalocyanine. Phys. Rev. B 2008, 77, 035133.

(32) Takeya, J.; Yamada, K.; Hara, K.; Shigeto, K.; Tsukagoshi, K.; Ikehata, S.; Aoyagi, Y. High-Density Electrostatic Carrier Doping in Organic Single-Crystal Transistors with Polymer Gel Electrolyte. Appl. Phys. Lett. 2006, 88, 2102.

(33) Gregg, B. A.; Cormier, R. A. Doping Molecular Semiconductors: N-Type Doping of a Liquid Crystal Perylene Diimide. J. Am. Chem. Soc. 2001, 123, 7959-7960.

(34) Kittel, C. Introduction to Solid State Physics, 7th ed.; John Wiley \& Sons, Inc., 1996.

(35) Nitzan, A. Chemical Dynamics in Condensed Phases: Relaxation, Transfer and Reactions in Condensed Molecular Systems; Oxford University Press, 2006.

(36) Atkins, P.; de Paula, J. Physical Chemistry; Oxford University Press., 2006.

(37) Oberhofer, H.; Blumberger, J. Revisiting Electronic Couplings and Incoherent Hopping Models for Electron Transport in Crystalline C60 at Ambient Temperatures. Phys. Chem. Chem. Phys. 2012, 14, 13846-13852.

(38) Gajdos, F.; Oberhofer, H.; Dupuis, M.; Blumberger, J. On the Inapplicability of Electron-Hopping Models for 
the Organic Semiconductor Phenyl-C61Butyric Acid Methyl Ester (PCBM). J. Phys. Chem. Lett. 2013, 4, 1012-1017.

(39) Ahrenkiel, R.; Dunlavy, D.; Greenberg, D.; Schlupmann, J.; Hamaker, H.; MacMillan, H. Electron Mobility in pGaAs by Time of Flight. Appl. Phys. Lett. 1987, 51, 776-778.

(40) Campbell, I.; Smith, D.; Neef, C.; Ferraris, J. Consistent Time-of-Flight Mobility Measurements and Polymer LightEmitting Diode Current \{endash\} Voltage Characteristics. Appl. Phys. Lett. 1999, 74, 2809-2811.

(41) Campbell, A. J.; Bradley, D. D.; Antoniadis, H. Dispersive Electron Transport in an Electroluminescent Polyfluorene Copolymer Measured by the Current Integration Time-of-Flight Method. Appl. Phys. Lett. 2001, 79, 2133-2135.

(42) Choulis, S.; Nelson, J.; Kim, Y.; Poplavskyy, D.; Kreouzis, T.; Durrant, J.; Bradley, D. Investigation of Transport Properties in Polymer/Fullerene Blends Using Time-ofFlight Photocurrent Measurements. Appl. Phys. Lett. 2003, 83, 3812-3814.

(43) Iino, H.; ichi Hanna, J.; Bushby, R. J.; Movaghar, B.; Whitaker, B. J.; Cook, M. J. Very High Time-of-Flight Mobility in the Columnar Phases of a Discotic Liquid Crystal. Appl. Phys. Lett. 2005, 87, 132102.

(44) Spear, W. E. Drift Mobility Techniques for the Study of Electrical Transport Properties in Insulating Solids. J. NonCryst. Solids 1969, 1, 197-214.

(45) Lebedev, E.; Dittrich, T.; PetrovaKoch, V.; Karg, S.; Brütting, W. Charge Carrier Mobility in Poly (pphenylenevinylene) Studied by the Timeof-Flight Technique. Appl. Phys. Lett. 1997, 71, 2686-2688.
(46) Tiwari, S.; Greenham, N. Charge Mobility Measurement Techniques in Organic Semiconductors. Opt. Quant. Electron. 2009, 41, 69-89.

(47) Friedman, L.; Pollak, M. Hall Mobility Due to Hopping-Type Conduction in Disordered Systems. Philos. Mag. B 1978, 38, 173-189.

(48) Podzorov, V.; Menard, E.; Rogers, J.; Gershenson, M. Hall Effect in the Accumulation Layers on the Surface of Organic Semiconductors. Phys. Rev. Lett. 2005, 95, 226601.

(49) Shuttle, C. G.; Hamilton, R.; Nelson, J.; O'Regan, B. C.; Durrant, J. R. Measurement of Charge-Density Dependence of Carrier Mobility in an Organic Semiconductor Blend. Adv. Funct. Mater. 2010, 20, 698-702.

(50) An, Z.; Yu, J.; Jones, S. C.; Barlow, S.; Yoo, S.; Domercq, B.; Prins, P.; Siebbeles, L. D.; Kippelen, B.; Marder, S. High Electron Mobility in Room-Temperature Discotic Liquid-Crystalline Perylene Diimides. Adv. Mater. 2005, 17, 25802583 .

(51) Schouten, P. G.; Warman, J. M.; de Haas, M. P. Effect of Accumulated Radiation Dose on Pulse Radiolysis Conductivity Transients in a Mesomorphic Octa-n-alkoxy-substituted Phthalocyanine. J. Phys. Chem. 1993, 97, 98639870 .

(52) Mozer, A. J.; Dennler, G.; Sariciftci, N.; Westerling, M.; Pivrikas, A.; Österbacka, R.; Juška, G. TimeDependent Mobility and Recombination of the Photoinduced Charge Carriers in Conjugated Polymer/Fullerene Bulk Heterojunction Solar Cells. Phys. Rev. B 2005, 72, 035217.

(53) Juška, G.; Genevičius, K.; Nekrašas, N.; Sliaužys, G.; Dennler, G. Trimolecular Recombination in Polythiophene: 
Fullerene Bulk Heterojunction Solar Cells. Appl. Phys. Lett. 2008, 93, 369.

(54) Kubo, R.; Toda, M.; Hashitsume, N. Statistical Physics II. Nonequilibrium Statistical Mechanics; Springer Verlag: Berlin, 1991.

(55) Furo, I.; Dvinskikh, S. V. NMR Methods Applied to Anisotropic Diffusion. Magn. Reson. Chem. 2002, 40, S3-S14.

(56) Girifalco, L. A. Statistical Mechanics of Solids; OUP USA, 2003; Vol. 58.

(57) Frenkel, D.; Smit, B. Understanding Molecular Simulation: From Algorithms to Applications; Academic press, 2001; Vol. 1.

(58) Troisi, A.; Cheung, D. L.; Andrienko, D. Charge Transport in Semiconductors with Multiscale Conformational Dynamics. Phys. Rev. Lett. 2009, 102, 116602.

(59) Troisi, A.; Cheung, D. L. Transition from Dynamic to Static Disorder in OneDimensional Organic Semiconductors. J. Chem. Phys. 2009, 131, 014703.

(60) Su, W.; Schrieffer, J.; Heeger, A. J. Solitons in Polyacetylene. Phys. Rev. Lett. 1979, 42, 1698.

(61) Hannewald, K.; Stojanović, V.; Schellekens, J.; Bobbert, P.; Kresse, G.; Hafner, J. Theory of Polaron Bandwidth Narrowing in Organic Molecular Crystals. Phys. Rev. B 2004, 69, 075211.

(62) Fornari, R. P.; Troisi, A. Theory of Charge Hopping along a Disordered Polymer Chain. Phys. Chem. Chem. Phys. 2014, 16, 9997-10007.

(63) Zhugayevych, A.; Postupna, O.; Bakus II, R. C.; Welch, G. C.; Bazan, G. C.; Tretiak, S. Ab Initio Study of a Molecular Crystal for Photovoltaics: Light Absorption, Exciton and Charge Carrier Transport. J. Phys. Chem. C 2013, 117, 49204930.
(64) Keck, J. C. Variational Theory of Reaction Rates; Wiley Online Library, 1967.

(65) Miller, W. H. Quantum Mechanical Transition State Theory and a New Semiclassical Model for Reaction Rate Constants. J. Chem. Phys. 1974, 61, 18231834

(66) Mones, L.; Kulhánek, P.; Simon, I.; Laio, A.; Fuxreiter, M. The Energy Gap as a Universal Reaction Coordinate for the Simulation of Chemical Reactions. $J$. Phys. Chem. B 2009, 113, 7867-7873.

(67) Mones, L.; Csányi, G. Topologically Invariant Reaction Coordinates for Simulating Multistate Chemical Reactions. J. Phys. Chem. B 2012, 116, 14876-14885.

(68) Zusman, L. Outer-Sphere Electron Transfer in Polar Solvents. Chem. Phys. 1980, 49, 295-304.

(69) Warshel, A. Dynamics of Reactions in Polar Solvents. Semiclassical Trajectory Studies of Electron-Transfer and ProtonTransfer Reactions. J. Phys. Chem. 1982, 86, 2218-2224.

(70) Kakitani, T.; Mataga, N. Comprehensive Study on the Role of Coordinated Solvent Mode Played in Electron-Transfer Reactions in Polar Solutions. J. Phys. Chem. 1987, 91, 6277-6285.

(71) Kuharski, R. A.; Bader, J. S.; Chandler, D.; Sprik, M.; Klein, M. L.; Impey, R. W. Molecular Model for Aqueous Ferrous-Ferric Electron Transfer. J. Chem. Phys. 1988, 89, 3248-3257.

(72) Carter, E. A.; Hynes, J. T. SoluteDependent Solvent Force Constants for Ion Pairs and Neutral Pairs in a Polar Solvent. J. Phys. Chem. 1989, 93, 21842187.

(73) Tachiya, M. Relation Between the Electron-Transfer Rate and the Free Energy Change of Reaction. J. Phys. Chem. 1989, 93, 7050-7052. 
(74) King, G.; Warshel, A. Investigation of the Free Energy Functions for Electron Transfer Reactions. J. Chem. Phys. 1990, 93, 8682-8692.

(75) Tateyama, Y.; Blumberger, J.; Sprik, M.; Tavernelli, I. Density-Functional Molecular-Dynamics Study of the Redox Reactions of Two Anionic, Aqueous Transition-Metal Complexes. J. Chem. Phys. 2005, 122, 234505.

(76) Cheng, J.; Sulpizi, M.; Sprik, M. Redox Potentials and pKa for Benzoquinone from Density Functional Theory Based Molecular Dynamics. J. Chem. Phys. 2009, 131, 154504.

(77) Vuilleumier, R.; Tay, K. A.; Jeanmairet, G.; Borgis, D.; Boutin, A. Extension of Marcus Picture for Electron Transfer Reactions with Large Solvation Changes. J. Am. Chem. Soc. 2012, 134, 2067-2074.

(78) Stecher, T.; Reuter, K.; Oberhofer, H. First-Principles Free-Energy Barriers for Photoelectrochemical Surface Reactions: Proton Abstraction at TiO 2 (110). Phys. Rev. Lett. 2016, 117, 276001.

(79) Moens, J.; Seidel, R.; Geerlings, P.; Faubel, M.; Winter, B.; Blumberger, J. Energy Levels and Redox Properties of Aqueous Mn2+/3+ from Photoemission Spectroscopy and Density Functional Molecular Dynamics Simulation. J. Phys. Chem. B 2010, 114, 9173-9182.

(80) Seidel, R.; Faubel, M.; Winter, B.; Blumberger, J. Single-Ion Reorganization Free Energy of Aqueous Ru (bpy) $32+/ 3+$ and $\mathrm{Ru}$ (H2O) $62+/ 3+$ from Photoemission Spectroscopy and Density Functional Molecular Dynamics Simulation. J. Am. Chem. Soc. 2009, 131, 1612716137.

(81) Brunschwig, B. S.; Logan, J.; Newton, M. D.; Sutin, N. A Semiclassical
Treatment of Electron-Exchange Reactions. Application to the Hexaaquoiron (II)-Hexaaquoiron (III) System. J. Am. Chem. Soc. 1980, 102, 5798-5809.

(82) Newton, M. D. Quantum Chemical Probes of Electron-Transfer Kinetics: The Nature of Donor-Acceptor Interactions. Chem. Rev. 1991, 91, 767-792.

(83) Landau, L. D. A Theory of Energy Transfer on Collisions. Phys. Z. Sowjetunion 1932, 1, 88.

(84) Landau, L. D. A Theory of Energy Transfer on Collisions II. Phys. Z. Sowjetunion 1932, 2, 46.

(85) Zener, C. Non-Adiabatic Crossing of Energy Levels. Proc. R. Soc. London, Ser. A 1932, 137, 696-702.

(86) Spencer, J.; Scalfi, L.; Carof, A.; Blumberger, J. Confronting Surface Hopping Molecular Dynamics with Marcus Theory for a molecular donor-acceptor system. Farad. Discuss. 2016, 195, 215-236.

(87) McKenna, K. P.; Wolf, M. J.; Shluger, A. L.; Lany, S.; Zunger, A. Two-Dimensional Polaronic Behavior in the Binary Oxides m- HfO 2 and m- ZrO 2. Phys. Rev. Lett. 2012, 108, 116403.

(88) Blumberger, J.; McKenna, K. P. Constrained Density Functional Theory Applied to Electron Tunnelling between Defects in MgO. Phys. Chem. Chem. Phys. 2013, 15, 2184-2196.

(89) Marcus, R. A. On the Theory of Oxidation-Reduction Reactions Involving Electron Transfer. I. J. Chem. Phys. 1956, 24, 966-978.

(90) Marcus, R. A. Electron Transfer Reactions in Chemistry. Theory and Experiment. Rev. Mod. Phys. 1993, 65, 599610.

(91) Fuchs, A.; Steinbrecher, T.; Mommer, M. S.; Nagata, Y.; Elstner, M.; 
Lennartz, C. Molecular Origin of Differences in Hole and Electron Mobility in Amorphous Alq3-A Multiscale Simulation Study. Phys. Chem. Chem. Phys. 2012, 14, 4259-4270.

(92) Oberhofer, H.; Blumberger, J. Charge Constrained Density Functional Molecular Dynamics for Simulation of Condensed Phase Electron Transfer Reactions. J. Chem. Phys. 2009, 131, 064101.

(93) Spencer, J.; Gajdos, F.; Blumberger, J. FOB-SH: Fragment Orbital-Based Surface Hopping for Charge Carrier Transport in Organic and Biological Molecules and Materials. J. Chem. Phys. 2016, $145,064102$.

(94) Martinelli, N. G.; Idé, J.; SánchezCarrera, R. S.; Coropceanu, V.; Brédas, J.-L.; Ducasse, L.; Castet, F.; Cornil, J.; Beljonne, D. Influence of Structural Dynamics on Polarization Energies in Anthracene Single Crystals. J. Phys. Chem. $C$ 2010, 114, 20678-20685.

(95) Cohen, A. J.; Mori-Sánchez, P.; Yang, W. Insights into Current Limitations of Density Functional Theory. Science 2008, 321, 792-794.

(96) Valeev, E. F.; Coropceanu, V.; da Filho, D. A. S.; Salman, S.; Brédas, J.L. Effect of Electronic Polarization on Charge-Transport Parameters in Molecular Organic Semiconductors. J. Am. Chem. Soc. 2006, 128, 9882-9886.

(97) Ryno, S. M.; Lee, S. R.; Sears, J. S.; Risko, C.; Brédas, J.-L. Electronic Polarization Effects upon Charge Injection in Oligoacene Molecular Crystals: Description via a Polarizable Force Field. J. Phys. Chem. C 2013, 117, 13853-13860.

(98) Norton, J. E.; Brédas, J.-L. Polarization Energies in Oligoacene Semiconductor Crystals. J. Am. Chem. Soc. 2008, 130, 12377-12384.
(99) Friederich, P.; Symalla, F.; Meded, V.; Neumann, T.; Wenzel, W. Ab Initio Treatment of Disorder Effects in Amorphous Organic Materials: Toward Parameter Free Materials Simulation. J. Chem. Theory Comput. 2014, 10, 3720 3725 .

(100) Castet, F.; Ducasse, L.; Fritsch, A. From Organic Superconductors to DNA: Fragment Orbital-Based Model. Int. J. Quantum Chem. 2006, 106, 734-746.

(101) Castet, F.; Aurel, P.; Fritsch, A.; Ducasse, L.; Liotard, D.; Linares, M.; Cornil, J.; Beljonne, D. Electronic Polarization Effects on Charge Carriers in Anthracene: A Valence Bond Study. Phys. Rev. B 2008, 77, 115210.

(102) Nagata, Y. Polarizable Atomistic Calculation of Site Energy Disorder in Amorphous Alq3. Chem. Phys. Chem. 2010, 11, 474-479.

(103) Morita, A.; Kato, S. Ab Initio Molecular Orbital Theory on Intramolecular Charge Polarization: Effect of Hydrogen Abstraction on the Charge Sensitivity of Aromatic and Nonaromatic Species. $J$. Am. Chem. Soc. 1997, 119, 4021-4032.

(104) Kwiatkowski, J.; Nelson, J.; Li, H.; Bredas, J.; Wenzel, W.; Lennartz, C. Simulating Charge Transport in Tris (8hydroxyquinoline) Aluminium (Alq 3). Phys. Chem. Chem. Phys. 2008, 10, 1852-1858.

(105) Bässler, H. Localized States and Electronic Transport in Single Component Organic Solids with Diagonal Disorder. Phys. Status Solidi (b) 1981, 107, 9-54.

(106) Bässler, H. Charge Transport in Disordered Organic Photoconductors a Monte Carlo Simulation Study. Phys. Status Solidi (b) 1993, 175, 15-56.

(107) Eley, D.; Evans, M. Heats and Entropy Changes Accompanying the Solution of 
Ions in Water. Trans. Faraday Soc. 1938, 34, 1093-1112.

(108) Bader, J. S.; Cortis, C. M.; Berne, B. Solvation and Reorganization Energies in Polarizable Molecular and Continuum Solvents. J. Chem. Phys. 1997, 106, 2372-2387.

(109) Ayala, R.; Sprik, M. A Classical Point Charge Model Study of System Size Dependence of Oxidation and Reorganization Free Energies in Aqueous Solution. J. Phys. Chem. B 2008, 112, 257-269.

(110) Blumberger, J.; Lamoureux, G. Reorganization Free Energies and Quantum Corrections for a Model Electron SelfExchange Reaction: Comparison of Polarizable and Non-Polarizable Solvent Models. Mol. Phys. 2008, 106, 15971611 .

(111) Weaver, M. J.; Gennett, T. Influence of Solvent Reorientation Dynamics upon the Kinetics of Some Electron-Exchange Reactions. Chem. Phys. Lett. 1985, 113, 213-218.

(112) Nielson, R. M.; McManis, G. E.; Golovin, M. N.; Weaver, M. J. Solvent Dynamical Effects in Electron Transfer: Comparisons of Self-Exchange Kinetics for Cobaltocenium-Cobaltocene and Related Redox Couples with Theoretical Predictions. J. Phys. Chem. 1988, 92, 3441-3450.

(113) Reimers, J. R. A Practical Method for the use of Curvilinear Coordinates in Calculations of Normal-Mode-Projected Displacements and Duschinsky Rotation Matrices for Large Molecules. J. Chem. Phys. 2001, 115, 9103-9109.

(114) Closs, G. L.; Miller, J. R. Intramolecular Long-Distance Electron Transfer in Organic Molecules. Science 1988, 240, 440448.

(115) Gruhn, N. E.; da Filho, D. A. S.; Bill, T. G.; Malagoli, M.;
Coropceanu, V.; Kahn, A.; Brédas, J.-L. The Vibrational Reorganization Energy in Pentacene: Molecular Influences on Charge Transport. J. Am. Chem. Soc. 2002, 124, 7918-7919.

(116) Amashukeli, X.; Winkler, J. R.; Gray, H. B.; Gruhn, N. E.; Lichtenberger, D. L. Electron-Transfer Reorganization Energies of Isolated Organic Molecules. J. Phys. Chem. A 2002, 106, 7593-7598.

(117) Amashukeli, X.; Gruhn, N. E.; Lichtenberger, D. L.; Winkler, J. R.; Gray, H. B. Inner-Sphere Electron-Transfer Reorganization Energies of Zinc Porphyrins. J. Am. Chem. Soc. 2004, 126, 1556615571.

(118) Coropceanu, V.; Kwon, O.; Wex, B.; Kaafarani, B. R.; Gruhn, N. E.; Durivage, J. C.; Neckers, D. C.; Brédas, J.-L. Vibronic Coupling in Organic Semiconductors: The Case of Fused Polycyclic Benzene-Thiophene Structures. Chem. Eur. J. 2006, 12, 2073-2080.

(119) Tipmanee, V.; Oberhofer, H.; Park, M.; Kim, K. S.; Blumberger, J. Prediction of Reorganization Free Energies for Biological Electron Transfer: A Comparative Study of Ru-modified Cytochromes and a 4-helix Bundle Protein. J. Am. Chem. Soc. 2010, 132, 17032-17040.

(120) Laplante, J.-P.; Siebrand, W. NonArrhenius Behaviour of Transitions across Energy Barriers Due to Crossing of Potential Energy Surfaces. Chem. Phys. Lett. 1978, 59, 433-436.

(121) Jortner, J.; Ulstrup, J. Tunnelling in Low-Temperature Atom-Transfer Processes. Chem. Phys. Lett. 1979, 63, 236239 .

(122) Holstein, T. Studies of Polaron Motion: Part I. The Molecular-Crystal Model. Ann. Phys. 1959, 8, 325-342. 
(123) Holstein, T. Studies of Polaron Motion: Part II. The "Small" Polaron. Ann. Phys. 1959, 8, 343-389.

(124) Holstein, T. Quantal OccurrenceProbability Treatment of Small-Polaron Hopping. Philos. Mag. B 1978, 37, 49-62.

(125) Barbara, P. F.; Meyer, T. J.; Ratner, M. A. Contemporary Issues in Electron Transfer Research. J. Phys. Chem. 1996, 100, 13148-13168.

(126) Geng, Y.; Wu, S.-X.; Li, H.-B.; Tang, X.D.; Wu, Y.; Su, Z.-M.; Liao, Y. A Theoretical Discussion on the Relationships among Molecular Packings, Intermolecular Interactions, and Electron Transport Properties for Naphthalene Tetracarboxylic Diimide Derivatives. $J$. Mater. Chem. 2011, 21, 15558-15566.

(127) Lichten, W. Molecular Wave Functions and Inelastic Atomic Collisions. Physical Review 1967, 164, 131-142.

(128) Smith, F. T. Diabatic and Adiabatic Representations for Atomic Collision Problems. Physical Review 1969, 179, 111-123.

(129) Cave, R. J.; Newton, M. D. Generalization of the Mulliken-Hush Treatment for the Calculation of Electron Transfer Matrix Elements. Chem. Phys. Lett. 1996, 249, 15-19.

(130) Mead, C. A.; Truhlar, D. G. Conditions for the Definition of a Strictly Diabatic Electronic Basis for Molecular Systems. J. Chem. Phys. 1982, 77, 6090-6098.

(131) Domcke, W.; Stock, G. Theory of Ultrafast Nonadiabatic Excited-State Processes and their Spectroscopic Detection in Real Time. Adv. Chem. Phys. 1997, $1-169$.

(132) Oberhofer, H.; Blumberger, J. Insight into the Mechanism of the Ru2+-Ru3+ Electron Self-Exchange Reaction from
Quantitative Rate Calculations. Angew. Chem. Int. Ed. 2010, 49, 3631-3634.

(133) Kubas, A.; Hoffmann, F.; Heck, A.; Oberhofer, H.; Elstner, M.; Blumberger, J. Electronic Couplings for Molecular Charge Transfer: Benchmarking CDFT, FODFT, and FODFTB against High-Level Ab Initio Calculations. J. Chem. Phys. 2014, 140, 104105.

(134) Brédas, J.-L.; Calbert, J. P.; da Filho, D. S.; Cornil, J. Organic Semiconductors: A Theoretical Characterization of the Basic Parameters Governing Charge Transport. Proc. Natl. Acad. Sci. USA 2002, 99, 5804-5809.

(135) da Filho, D. A. S.; Kim, E.-G.; Brédas, J.-L. Transport Properties in the Rubrene Crystal: Electronic Coupling and Vibrational Reorganization Energy. Adv. Mater. 2005, 17, 1072-1076.

(136) Rangel, T.; Berland, K.; Sharifzadeh, S.; Brown-Altvater, F.; Lee, K.; Hyldgaard, P.; Kronik, L.; Neaton, J. B. Structural and Excited-State Properties of Oligoacene Crystals from First Principles. Phys. Rev. $B$ 2016, 93, 115206.

(137) Worth, G.; Robb, M. Applying Direct Molecular Dynamics to Non-Adiabatic Systems. Adv. Chem. Phys. 2003, 124, 355-432.

(138) Pacher, T.; Cederbaum, L. S.; Köppel, H. Approximately Diabatic States from Block Diagonalization of the Electronic Hamiltonian. J. Chem. Phys. 1988, 89, 7367-7381.

(139) Baer, M. Adiabatic and Diabatic Representations for Atom-Molecule Collisions: Treatment of the Collinear Arrangement. Chem. Phys. Lett. 1975, 35, 112-118.

(140) Baer, M. Topological Effects in Molecular Systems: An Attempt Towards a Complete Theory. Chem. Phys. 2000, 259, 123-147. 
(141) Macias, A.; Riera, A. Calculation of Diabatic States from Molecular Properties. J. Phys. B 1978, 11, L489-L492.

(142) Werner, H.-J.; Meyer, W. MCSCF Study of the Avoided Curve Crossing of the Two Lowest $1 \Sigma+$ States of LiF. J. Chem. Phys. 1981, 74, 5802-5807.

(143) Domcke, W.; Woywod, C. Direct Construction of Diabatic States in the CASSCF Approach. Application to the Conical Intersection of the 1A2 and 1B1 Excited States of Ozone. Chem. Phys. Lett. 1993, 216, 362-368.

(144) Köppel, H.; Gronki, J.; Mahapatra, S. Construction Scheme for Regularized Diabatic States. J. Chem. Phys. 2001, 115, 2377-2388.

(145) Kubas, A.; Gajdos, F.; Heck, A.; Oberhofer, H.; Elstner, M.; Blumberger, J. Electronic Couplings for Molecular Charge Transfer: Benchmarking CDFT, FODFT and FODFTB against High-Level Ab Initio Calculations. II. Phys. Chem. Chem. Phys. 2015, 17, 14342-14354.

(146) Ramos, P.; Mankarious, M.; Pavanello, M. Practical Aspects of Computational Chemistry IV; Springer, 2016; pp 103-134.

(147) Gillet, N.; Berstis, L.; Wu, X.; Gajdos, F.; Heck, A.; de la Lande, A.; Blumberger, J.; Elstner, M. Electronic Coupling Calculations for Bridge-Mediated Charge Transfer Using Constrained Density Functional Theory (CDFT) and Effective Hamiltonian Approaches at the Density Functional Theory (DFT) and Fragment-Orbital Density Functional Tight Binding (FODFTB) Level. J. Chem. Theory Comput. 2016, 12, 4793-4805.

(148) Mulliken, R. S. Molecular Compounds and their Spectra. II. J. Am. Chem. Soc. 1952, 74, 811-824.
(149) Hush, N. Homogeneous and Heterogeneous Optical and Thermal Electron Transfer. Electrochim. Acta 1968, 13, 1005-1023.

(150) Reimers, J. R.; Hush, N. S. Electronic Properties of Transition-Metal Complexes Determined from Electroabsorption (Stark) Spectroscopy. 2. Mononuclear Complexes of Ruthenium(II). J. Phys. Chem. 1991, 95, 9773-9781.

(151) Cave, R. J.; Newton, M. D. Calculation of Electronic Coupling Matrix Elements for Ground and Excited State Electron Transfer Reactions: Comparison of the Generalized Mulliken-Hush and Block Diagonalization Methods. J. Chem. Phys. 1997, 106, 9213-9226.

(152) Wu, Q.; Voorhis, T. V. Direct Optimization Method to Study Constrained Systems within Density-Functional Theory. Phys. Rev. A 2005, 72, 024502.

(153) Mukherji, A.; Karplus, M. Constrained Molecular Wavefunctions: HF Molecule. J. Chem. Phys. 1963, 38, 44-48.

(154) Dederichs, P.; Blügel, S.; Zeller, R.; Akai, H. Ground States of Constrained Systems: Application to Cerium Impurities. Phys. Rev. Lett. 1984, 53, 25122515 .

(155) Akai, H.; Blügel, S.; Zeller, R.; Dederichs, P. Isomer Shifts and their Relation to Charge Transfer in Dilute Fe Alloys. Phys. Rev. Lett. 1986, 56, $2407-$ 2410 .

(156) Hybertsen, M. S.; Schlüter, M.; Christensen, N. E. Calculation of CoulombInteraction Parameters for $\mathrm{La} 2 \mathrm{CuO} 4$ Using a Constrained-Density-Functional Approach. Phys. Rev. B 1989, 39, 90289041.

(157) Zhang, Z.; Satpathy, S. Electron States, Magnetism, and the Verwey Transition in Magnetite. Phys. Rev. B 1991, 44 , 13319 . 
(158) Wesolowski, T. A.; Warshel, A. Frozen Density Functional Approach for Ab Initio Calculations of Solvated Molecules. J. Phys. Chem. 1993, 97, 8050-8053.

(159) Hong, G.; Strajbl, M.; Wesolowski, T. A.; Warshel, A. Constraining the Electron Densities in DFT Method as an Effective Way for Ab Initio Studies of MetalCatalyzed Reactions. J. Comput. Chem. 2000, 21, 1554-1561.

(160) Behler, J.; Delley, B.; Lorenz, S.; Reuter, K.; Scheffler, M. Dissociation of $\mathrm{O} 2$ at $\mathrm{Al}$ (111): The Role of Spin Selection Rules. Phys. Rev. Lett. 2005, 94, 036104.

(161) Behler, J.; Delley, B.; Reuter, K.; Scheffler, M. Nonadiabatic PotentialEnergy Surfaces by Constrained DensityFunctional Theory. Phys. Rev. B 2007, 75, 115409.

(162) Wu, Q.; Voorhis, T. V. Extracting Electron Transfer Coupling Elements from Constrained Density Functional Theory. J. Chem. Phys. 2006, 125, 164105.

(163) Wu, Q.; Voorhis, T. V. Constrained Density Functional Theory and its Application in Long-Range Electron Transfer. $J$. Chem. Theory Comput. 2006, 2, 765774 .

(164) Wu, Q.; Voorhis, T. V. Direct Calculation of Electron Transfer Parameters through Constrained Density Functional Theory. J. Phys. Chem. A 2006, 110, 9212-9218.

(165) Oberhofer, H.; Blumberger, J. Electronic Coupling Matrix Elements from Charge Constrained Density Functional Theory Calculations Using a Plane Wave Basis Set. J. Chem. Phys. 2010, 133, 244105.

(166) Mulliken, R. S. Electronic Population Analysis on LCAO-MO Molecular Wave Functions. I. J. Chem. Phys. 1955, 23, 1833-1840.
(167) Hirshfeld, F. Bonded-Atom Fragments for Describing Molecular Charge Densities. Theor. Chem. Acc. 1977, 44, 129 138.

(168) Bader, R. F. Atoms in Molecules; Wiley Online Library, 1990.

(169) Rezac, J.; Lévy, B.; Demachy, I.; de la Lande, A. Robust and Efficient Constrained DFT Molecular Dynamics Approach for Biochemical Modeling. $J$. Chem. Theory Comput. 2012, 8, 418427.

(170) Řezáč, J.; de la Lande, A. Robust, BasisSet Independent Method for the Evaluation of Charge-Transfer Energy in Noncovalent Complexes. J. Chem. Theory Comput. 2015, 11, 528-537.

(171) Rapacioli, M.; Spiegelman, F. Modelling Singly Ionized Coronene Clusters. Eur. Phys. J. D 2009, 52, 55-58.

(172) Rapacioli, M.; Spiegelman, F.; Scemama, A.; Mirtschink, A. Modeling Charge Resonance in Cationic Molecular Clusters: Combining DFT-Tight Binding with Configuration Interaction. $J$. Chem. Theory Comput. 2011, 7, 44-55.

(173) Rapacioli, M.; Simon, A.; Dontot, L.; Spiegelman, F. Extensions of DFTB to Investigate Molecular Complexes and Clusters. Phys. Status Solidi (b) 2012, 249, 245-258.

(174) Porezag, D.; Frauenheim, T.; Köhler, T.; Seifert, G.; Kaschner, R. Construction of Tight-Binding-Like Potentials on the Basis of Density-Functional Theory: Application to Carbon. Phys. Rev. B 1995, 51, 12947-12957.

(175) Seifert, G.; Porezag, D.; Frauenheim, T. Calculations of Molecules, Clusters, and Solids with a Simplified LCAO-DFTLDA Scheme. Int. J. Quantum Chem. 1996, 58, 185-192. 
(176) Kubař, T.; Woiczikowski, P. B.; Cuniberti, G.; Elstner, M. Efficient Calculation of Charge-Transfer Matrix Elements for Hole Transfer in DNA. J. Phys. Chem. B 2008, 112, 7937-7947.

(177) Senthilkumar, K.; Grozema, F.; Bickelhaupt, F.; Siebbeles, L. Charge Transport in Columnar Stacked Triphenylenes: Effects of Conformational Fluctuations on Charge Transfer Integrals and Site Energies. J. Chem. Phys. 2003, 119, 9809-9817.

(178) Li, H.; Brédas, J.-L.; Lennartz, C. FirstPrinciples Theoretical Investigation of the Electronic Couplings in Single Crystals of Phenanthroline-based Organic Semiconductors. J. Chem. Phys. 2007, 126, 164704.

(179) Schober, C.; Reuter, K.; Oberhofer, H. Critical Analysis of Fragment-Orbital DFT Schemes for the Calculation of Electronic Coupling Values. J. Chem. Phys. 2016, $144,054103$.

(180) Baumeier, B.; Kirkpatrick, J.; Andrienko, D. Density-Functional Based Determination of Intermolecular Charge Transfer Properties for Large-Scale Morphologies. Phys. Chem. Chem. Phys. 2010, 12, 11103-11113.

(181) Sutton, C.; Sears, J. S.; Coropceanu, V.; Bredas, J.-L. Understanding the Density Functional Dependence of DFTCalculated Electronic Couplings in Organic Semiconductors. J. Phys. Chem. Lett. 2013, 4, 919-924.

(182) Lemaur, V.; da Filho, D. A. S.; Coropceanu, V.; Lehmann, M.; Geerts, Y.; Piris, J.; Debije, M. G.; van de Craats, A. M.; Senthilkumar, K.; Siebbeles, L. D. A. et al. Charge Transport Properties in Discotic Liquid Crystals: A Quantum-Chemical Insight into Structure-Property Relationships. J. Am. Chem. Soc. 2004, 126, 3271-3279.
(183) Woiczikowski, P. B.; Kubař, T.; Gutiérrez, R.; Cuniberti, G.; Elstner, M. Structural Stability Versus Conformational Sampling in Biomolecular Systems: Why is the Charge Transfer Efficiency in G4-DNA Better than in Double-Stranded DNA? J. Chem. Phys. 2010, 133, 035103.

(184) Wolter, M.; Woiczikowski, P. B.; Elstner, M.; Kubař, T. Response of the Electric Conductivity of Double-Stranded DNA on Moderate Mechanical Stretching Stresses. Phys. Rev. B 2012, 85, 075101.

(185) Wolter, M.; Elstner, M.; Kubař, T. Charge Transport in Desolvated DNA. J. Chem. Phys. 2013, 139, 125102.

(186) Heck, A.; Woiczikowski, P. B.; Kubař, T.; Giese, B.; Elstner, M.; Steinbrecher, T. B. Charge Transfer in Model Peptides: Obtaining Marcus Parameters from Molecular Simulation. J. Phys. Chem. B 2012, 116, 2284-2293.

(187) Lüdemann, G.; Woiczikowski, P. B.; Kubař, T.; Elstner, M.; Steinbrecher, T. B. Charge Transfer in E. Coli DNA Photolyase: Understanding Polarization and Stabilization Effects via QM/MM Simulations. J. Phys. Chem. B 2013, 117, 10769-10778.

(188) Heck, A.; Kranz, J. J.; Kubar, T.; Elstner, M. Multi-Scale Approach to NonAdiabatic Charge Transport in HighMobility Organic Semiconductors. J. Chem. Theory Comput. 2015, 11, 50685082 .

(189) Heck, A.; Kranz, J. J.; Elstner, M. Simulation of Temperature-Dependent Charge Transport in Organic Semiconductors with Various Degrees of Disorder. J. Chem. Theory Comput. 2016, 12, 3087-3096.

(190) Woiczikowski, P. B.; Steinbrecher, T.; Kubař, T.; Elstner, M. Nonadiabatic 
QM/MM Simulations of Fast Charge Transfer in Escherichia Coli DNA Photolyase. J. Phys. Chem. B 2011, 115, 9846-9863.

(191) Ridley, J.; Zerner, M. An Intermediate Neglect of Differential Overlap Technique for Spectroscopy: Pyrrole and the Azines. Theor. Chim. Acta 1973, 32, $111-134$.

(192) Kirkpatrick, J. An Approximate Method for Calculating Transfer Integrals Based on the ZINDO Hamiltonian. Int. J. Quantum Chem. 2008, 108, 51-56.

(193) Nelson, J.; Kwiatkowski, J. J.; Kirkpatrick, J.; Frost, J. M. Modeling Charge Transport in Organic Photovoltaic Materials. Acc. Chem. Res. 2009, 42, 17681778 .

(194) Akimov, A. V. Nonadiabatic Molecular Dynamics with Tight-Binding Fragment Molecular Orbitals. J. Chem. Theory Comput. 2016, 12, 5719-5736.

(195) Pavanello, M.; Neugebauer, J. Modelling Charge Transfer Reactions with the Frozen Density Embedding Formalism. J. Chem. Phys. 2011, 135, 234103.

(196) Wesolowski, T. A.; Weber, J. KohnSham Equations with Constrained Electron Density: An Iterative Evaluation of the Ground-State Electron Density of Interacting Molecules. Chem. Phys. Lett. 1996, $248,71-76$.

(197) Jacob, C. R.; Neugebauer, J.; Visscher, L. A Flexible Implementation of Frozen-Density Embedding for Use in Multilevel Simulations. J. Comput. Chem. 2008, 29, 1011-1018.

(198) Perdew, J. P.; Burke, K.; Ernzerhof, M. Generalized Gradient Approximation Made Simple. Phys. Rev. Lett. 1996, 77, 3865-3868.

(199) Blum, V.; Gehrke, R.; Hanke, F.; Havu, P.; Havu, V.; Ren, X.; Reuter, K.;
Scheffler, M. Ab Initio Molecular Simulations with Numeric Atom-Centered Orbitals. Comp. Phys. Commun. 2009, 180, 2175-2196.

(200) Wesołowski, T. A. Application of the DFT-based Embedding Scheme Using an Explicit Functional of the Kinetic Energy to Determine the Spin Density of $\mathrm{Mg}+$ Embedded in Ne and Ar Matrices. Chem. Phys. Lett. 1999, 311, 87-92.

(201) Neugebauer, J.; Louwerse, M. J.; Baerends, E. J.; Wesolowski, T. A. The Merits of the Frozen-Density Embedding Scheme to Model Solvatochromic Shifts. J. Chem. Phys. 2005, 122, 094115.

(202) Solovyeva, A.; Pavanello, M.; Neugebauer, J. Spin Densities from Subsystem Density-Functional Theory: Assessment and Application to a Photosynthetic Reaction Center Complex Model. J. Chem. Phys. 2012, 136, 194104.

(203) Pavanello, M.; Van Voorhis, T.; Visscher, L.; Neugebauer, J. An Accurate and Linear-Scaling Method for Calculating Charge-Transfer Excitation Energies and Diabatic Couplings. J. Chem. Phys. 2013, 138, 054101.

(204) Ramos, P.; Papadakis, M.; Pavanello, M. Performance of Frozen Density Embedding for Modeling Hole Transfer Reactions. J. Phys. Chem. B 2015, 119, $7541-7557$.

(205) Solovyeva, A.; Pavanello, M.; Neugebauer, J. Describing Long-Range ChargeSeparation Processes with Subsystem Density-Functional Theory. J. Chem. Phys. 2014, 140, 164103.

(206) Thom, A. J. W.; Head-Gordon, M. Hartree-Fock Solutions as a Quasidiabatic Basis for Nonorthogonal Configuration Interaction. J. Chem. Phys. 2009, 131,124113

(207) Kondov, I.; Cízek, M.; Benesch, C.; Wang, H.; Thoss, M. Quantum Dynamics 
of Photoinduced Electron-Transfer Reactions in Dye-Semiconductor Systems: First-Principles Description and Application to Coumarin 343-TiO2. J. Phys. Chem. C 2007, 111, 11970-11981.

(208) Priyadarshy, S.; Skourtis, S. S.; Risser, S. M.; Beratan, D. N. BridgeMediated Electronic Interactions: Differences Between Hamiltonian and Green Function Partitioning in a NonOrthogonal Basis. J. Chem. Phys. 1996, 104, 9473-9481.

(209) Kurnikov, I. V.; Beratan, D. N. Abinitio Based Effective Hamiltonians for LongRange Electron Transfer: Hartree-Fock Analysis. J. Chem. Phys. 1996, 105, 9561-9573.

(210) Galperin, M.; Toledo, S.; Nitzan, A. Numerical Computation of Tunneling Fluxes. J. Chem. Phys. 2002, 117, 10817-10826.

(211) Löwdin, P. O. On the Non-Orthogonality problem Connected with the Use of Atomic Wave Functions in the Theory of Molecules and Crystals. J. Chem. Phys. 1950, 18, 365-375.

(212) Mayer, I. On Löwdin's Method of Symmetric Orthogonalization. Int. J. Quantum Chem. 2002, 90, 63-65.

(213) Aiken, J. G.; Erdos, J. A.; Goldstein, J. A. On Löwdin Orthogonalization. Int. J. Quantum Chem. 1980, 18, 1101-1108.

(214) Prucker, V.; Rubio-Pons, O.; Bockstedte, M.; Wang, H.; Coto, P. B.; Thoss, M. Dynamical Simulation of Electron Transfer Processes in Alkanethiolate Self-Assembled Monolayers at the $\mathrm{Au}$ (111) Surface. J. Phys. Chem. C 2013, 117, 25334-25342.

(215) Li, J.; Nilsing, M.; Kondov, I.; Wang, H.; Persson, P.; Lunell, S.; Thoss, M. Dynamical Simulation of Photoinduced Electron Transfer Reactions in Dye-
Semiconductor Systems with Different Anchor Groups. J. Phys. Chem. C 2008, 112, 12326-12333.

(216) Gajdos, F.; Valner, S.; Hoffmann, F.; Spencer, J.; Breuer, M.; Kubas, A.; Dupuis, M.; Blumberger, J. Ultrafast Estimation of Electronic Couplings for Electron Transfer between $\pi$-conjugated Organic Molecules. J. Chem. Theory Comput. 2014, 10, 4653-4660.

(217) Geng, H.; Zheng, X.; Shuai, Z.; Zhu, L.; Yi, Y. Understanding the Charge Transport and Polarities in Organic DonorAcceptor Mixed-Stack Crystals: Molecular Insights from the Super-Exchange Couplings. Adv. Mater. 2015, 27, 14431449 .

(218) Ramos, P.; Pavanello, M. Quantifying Environmental Effects on the Decay of Hole Transfer Couplings in Biosystems. J. Chem. Theory Comput. 2014, 10, 2546-2556.

(219) Siddarth, P.; Marcus, R. Correlation between Theory and Experiment in Electron-Transfer Reactions in Proteins: Electronic Couplings in Modified $\mathrm{Cy}$ tochrome c and Myoglobin Derivatives. J. Phys. Chem. 1993, 97, 13078-13082.

(220) Gruschus, J. M.; Kuki, A. New Hamiltonian Model for Long-Range Electronic Superexchange in Complex Molecular Structures. J. Phys. Chem. 1993, 97, 5581-5593.

(221) Beeler, J. R. Displacement Spikes in Cubic Metals. I. $\alpha$-Iron, Copper, and Tungsten. Phys. Rev. 1966, 150, 470-487.

(222) Liu, D.-J.; Garcia, A.; Wang, J.; Ackerman, D. M.; Wang, C.-J.; Evans, J. W. Kinetic Monte Carlo Simulation of Statistical Mechanical Models and CoarseGrained Mesoscale Descriptions of Catalytic Reaction-Diffusion Processes: 1D Nanoporous and 2D Surface Systems. Chem. Rev. 2015, 115, 5979-6050. 
(223) Reuter, K. Ab Initio Thermodynamics and First-Principles Microkinetics for Surface Catalysis. Catal. Lett. 2016, 146, 541-563.

(224) Baumeier, B.; Stenzel, O.; Poelking, C.; Andrienko, D.; Schmidt, V. Stochastic Modeling of Molecular Charge Transport Networks. Phys. Rev. B 2012, 86, 184202 .

(225) Voter, A. F. In Radiation Effects in Solids; Sickafus, K. E., Kotamin, E. A., Uberuaga, B. P., Eds.; Springer, 2007; pp $1-23$.

(226) Vissenberg, M. C. J. M.; Matters, M. Theory of the Field-Effect Mobility in Amorphous Organic Transistors. Phys. Rev. B 1998, 5\%, 12964-12967.

(227) Fichthorn, K. A.; Scheffler, M. Island Nucleation in Thin-Film Epitaxy: A FirstPrinciples Investigation. Phys. Rev. Lett. 2000, 84, 5371-5374.

(228) Yu, Z. G.; Smith, D. L.; Saxena, A.; Martin, R. L.; Bishop, A. R. Molecular Geometry Fluctuations and FieldDependent Mobility in Conjugated Polymers. Phys. Rev. B 2001, 63, 085202.

(229) Fähnle, M.; Drautz, R.; Lechermann, F.; Singer, R.; Diaz-Ortiz, A.; Dosch, H. Thermodynamic Properties from AbInitio Calculations: New Theoretical Developments, and Applications to Various Materials Systems. Phys. Status Solidi (b) 2005, 242, 1159-1173.

(230) Zhang, Y.; Blum, V.; Reuter, K. Accuracy of First-Principles Lateral Interactions: Oxygen at $\mathrm{Pd}(100)$. Phys. Rev. B 2007, 75, 235406.

(231) Lorenzi, J. M.; Matera, S.; Reuter, K. Synergistic Inhibition of Oxide Formation in Oxidation Catalysis: A FirstPrinciples Kinetic Monte Carlo Study of $\mathrm{NO}+\mathrm{CO}$ Oxidation at $\mathrm{Pd}(100)$. ACS Catal. 2016, 6, 5191-5197.
(232) Idé, J.; Fazzi, D.; Casalegno, M.; Meille, S. V.; Raos, G. Electron Transport in Crystalline PCBM-like Fullerene Derivatives: a Comparative Computational Study. J. Mater. Chem. C 2014, 2, 7313-7325.

(233) Di Motta, S.; Di Donato, E.; Negri, F.; Orlandi, G.; Fazzi, D.; Castiglioni, C. Resistive Molecular Memories: Influence of Molecular Parameters on the Electrical Bistability. J. Am. Chem. Soc. 2009, 131, 6591-6598.

(234) Huong, V. T. T.; Tai, T. B.; Nguyen, M. T. Theoretical Design of n-type Organic Semiconducting Materials Containing Thiazole and Oxazole Frameworks. J. Phys. Chem. A 2014, 118, 3335-3343.

(235) McMahon, D. P.; Troisi, A. Evaluation of the External Reorganization Energy of Polyacenes. J. Phys. Chem. Lett. 2010, 1, 941-946.

(236) Cornil, J.; Verlaak, S.; Martinelli, N.; Mityashin, A.; Olivier, Y.; Van Regemorter, T.; D'Avino, G.; Muccioli, L.; Zannoni, C.; Castet, F. Exploring the Energy Landscape of the Charge Transport Levels in Organic Semiconductors at the Molecular Scale. Acc. Chem. Res. 2012, 46, 434-443.

(237) Athanasopoulos, S.; Kirkpatrick, J.; Martínez, D.; Frost, J. M.; Foden, C. M.; Walker, A. B.; Nelson, J. Predictive Study of Charge Transport in Disordered Semiconducting Polymers. Nano lett. 2007, 7, 1785-1788.

(238) Pershin, A.; Donets, S.; Baeurle, S. A. A New Multiscale Modeling Method for Simulating the Loss Processes in Polymer Solar Cell Nanodevices. J. Chem. Phys. 2012, 136, 194102.

(239) Walker, A. B. Multiscale Modeling of Charge and Energy Transport in Organic Light-Emitting Diodes and Pho- 
tovoltaics. Proc. IEEE 2009, 97, 15871596.

(240) Jacoboni, C.; Lugli, P. The Monte Carlo Method for Semiconductor Device Simulation; Springer, 1989; pp 6-103.

(241) LeBlanc Jr, O. H. Band Structure and Transport of Holes and Electrons in Anthracene. J. Chem. Phys. 1961, 35, $1275-1280$.

(242) Wang, L.; Beljonne, D. Flexible Surface Hopping Approach to Model the Crossover from Hopping to Band-like Transport in Organic Crystals. J. Phys. Chem. Lett. 2013, 4, 1888-1894.

(243) Blülle, B.; Troisi, A.; Häusermann, R.; Batlogg, B. Charge Transport Perpendicular to the High Mobility Plane in Organic Crystals: Bandlike Temperature Dependence Maintained Despite Hundredfold Anisotropy. Phys. Rev. B 2016, 93, 035205.

(244) Parker, S. P. McGraw-Hill Encyclopedia of Physics; McGraw-Hill Book Company, New York, NY, 1983.

(245) Jacoboni, C.; Reggiani, L. The Monte Carlo Method for the Solution of Charge Transport in Semiconductors with Application to Covalent Materials. Rev. Mod. Phys. 1983, 55, 645-705.

(246) Ziman, J. M. Principles of the Theory of Solids; Cambridge university press, 1972.

(247) Herring, C.; Vogt, E. Transport and Deformation-Potential Theory for ManyValley Semiconductors with Anisotropic Scattering. Phys. Rev. 1956, 101, 944961.

(248) Askerov, B. M. Electron Transport Phenomena in Semiconductors; World Scientific, 1994; Vol. 394.

(249) Xi, J.; Long, M.; Tang, L.; Wang, D.; Shuai, Z. First-Principles Prediction of
Charge Mobility in Carbon and Organic Nanomaterials. Nanoscale 2012, 4, 4348-4369.

(250) Szulczewski, G.; Sanvito, S.; Coey, M. A Spin of their Own. Nat. Mater. 2009, 8, 693-695.

(251) Katz, J. L.; Rice, S. A.; il Choi, S.; Jortner, J. On the Excess Electron and Hole Band Structures and Carrier Mobility in Naphthalene, Anthracene, and Several Polyphenyls. J. Chem. Phys. 1963, 39, 1683-1697.

(252) Cheng, Y.; Silbey, R.; da Filho, D. A. S.; Calbert, J. P.; Cornil, J.; Brédas, J.-L. Three-Dimensional Band Structure and Bandlike Mobility in Oligoacene Single Crystals: A Theoretical Investigation. J. Chem. Phys. 2003, 118, 3764-3774.

(253) Troisi, A.; Orlandi, G. Band Structure of the Four Pentacene Polymorphs and Effect on the Hole Mobility at Low Temperature. J. Phys. Chem. B 2005, 109, 1849-1856.

(254) Kobayashi, H.; Kobayashi, N.; Hosoi, S.; Koshitani, N.; Murakami, D.; Shirasawa, R.; Kudo, Y.; Hobara, D.; Tokita, Y.; Itabashi, M. Hopping and Band Mobilities of Pentacene, Rubrene, and 2, 7-dioctyl[1]benzothieno[3, 2b][1]benzothiophene (C8-BTBT) from First Principle Calculations. J. Chem. Phys. 2013, 139, 014707.

(255) Bardeen, J.; Shockley, W. Deformation Potentials and Mobilities in Non-Polar Crystals. Phys. Rev. 1950, 80, 72-80.

(256) Harrison, W. A. Scattering of Electrons by Lattice Vibrations in Nonpolar Crystals. Phys. Rev. 1956, 104, 1281-1290.

(257) Fratini, S.; Ciuchi, S. Bandlike Motion and Mobility Saturation in Organic Molecular Semiconductors. Phys. Rev. Lett. 2009, 103, 266601. 
(258) Fratini, S.; Mayou, D.; Ciuchi, S. The Transient Localization Scenario for Charge Transport in Crystalline Organic Materials. Adv. Funct. Mater. 2016, 2292-2315.

(259) Northrup, J. Two-Dimensional Deformation Potential Model of Mobility in Small Molecule Organic Semiconductors. Appl. Phys. Lett. 2011, 99, 062111.

(260) Beleznay, F.; Bogár, F.; Ladik, J. Charge Carrier Mobility in Quasi-OneDimensional Systems: Application to a Guanine Stack. J. Chem. Phys. 2003, 119, 5690-5695.

(261) Price, P. Two-Dimensional Electron Transport in Semiconductor Layers. I. Phonon Scattering. Ann. Phys. 1981, 133, 217-239.

(262) Walukiewicz, W.; Ruda, H.; Lagowski, J.; Gatos, H. Electron Mobility in Modulation-Doped Heterostructures. Phys. Rev. B 1984, 30, 4571-4582.

(263) Fishman, G. Phonon-Limited Mobility in a Quasi-One-Dimensional Semiconductor. Phys. Rev. B 1987, 36, 7448-7456.

(264) Takagi, S.; Hoyt, J. L.; Welser, J. J.; Gibbons, J. F. Comparative Study of Phonon-Limited Mobility of TwoDimensional Electrons in Strained and Unstrained Si Metal-OxideSemiconductor Field-Effect Transistors. J. Appl. Phys. 1996, 80, 1567-1577.

(265) Togo, A.; Chaput, L.; Tanaka, I.; Hug, G. First-Principles Phonon Calculations of Thermal Expansion in $\mathrm{Ti} 3 \mathrm{SiC} 2$, Ti 3 AlC 2, and Ti 3 GeC 2. Phys. Rev. B 2010, 81, 174301.

(266) Giuggioli, L.; Andersen, J. D.; Kenkre, V. Mobility Theory of Intermediate-Bandwidth Carriers in Organic Crystals: Scattering by Acoustic and Optical Phonons. Phys. Rev. B 2003, 67, 045110.
(267) Li, Y.; Yi, Y.; Coropceanu, V.; Brédas, J.-L. Optical Conductivity and Optical Effective Mass in a HighMobility Organic Semiconductor: Implications for the Nature of Charge Transport. Phys. Rev. B 2014, 90, 245112.

(268) Yang, H.; Gajdos, F.; Blumberger, J. Intermolecular Charge Transfer Parameters, Electron-Phonon Couplings, and the Validity of Polaron Hopping Models in Organic Semiconducting Crystals: Rubrene, Pentacene, and C60. J. Phys. Chem. C 2017, 121, 7689-7696.

(269) Troisi, A. The Speed Limit for Sequential Charge Hopping in Molecular Materials. Org. Electron. 2011, 12, 1988-1991.

(270) Dong, H.; Fu, X.; Liu, J.; Wang, Z.; $\mathrm{Hu}$, W. 25th Anniversary Article: Key Points for High-Mobility Organic FieldEffect Transistors. Adv. Mater. 2013, 25, 6158-6183.

(271) Gershenson, M.; Podzorov, V.; Morpurgo, A. Colloquium: Electronic Transport in Single-Crystal Organic Transistors. Rev. Mod. Phys. 2006, 78, 973-989.

(272) Mott, N. F.; Davis, E. A. Electronic Processes in Non-Crystalline Materials; OUP Oxford, 2012.

(273) Street, R.; Northrup, J.; Salleo, A. Transport in Polycrystalline Polymer ThinFilm Transistors. Phys. Rev. B 2005, 71, 165202.

(274) Troisi, A.; Orlandi, G. Dynamics of the Intermolecular Transfer Integral in Crystalline Organic Semiconductors. J. Phys. Chem. A 2006, 110, 4065-4070.

(275) Emin, D. Phonon-Assisted Transition Rates I. Optical-Phonon-Assisted Hopping in Solids. Adv. Phys. 1975, 24, 305348.

(276) De Raedt, H.; Lagendijk, A. Numerical Study of Holstein's Molecular-Crystal Model: Adiabatic Limit and Influence of 
Phonon Dispersion. Phys. Rev. B 1984, 30, 1671-1678.

(277) Kenkre, V.; Andersen, J. D.; Dunlap, D.; Duke, C. Unified Theory of the Mobilities of Photoinjected Electrons in Naphthalene. Phys. Rev. Lett. 1989, 62, 11651168 .

(278) Wu, M.; Conwell, E. Transport in $\alpha$ sexithiophene Films. Chem. Phys. Lett. 1997, 266, 363-367.

(279) Hannewald, K.; Bobbert, P. Ab Initio Theory of Charge-Carrier Conduction in Ultrapure Organic Crystals. Appl. Phys. Lett. 2004, 85, 1535-1537.

(280) Friedman, L. Electron-Phonon Interaction in Organic Molecular Crystals. Phys. Rev. 1965, 140, A1649-A1667.

(281) Munn, R.; Silbey, R. Theory of Electronic Transport in Molecular Crystals. II. Zeroth Order States Incorporating Nonlocal Linear Electron-Phonon Coupling. J. Chem. Phys. 1985, 83, 18431853.

(282) Troisi, A. Prediction of the Absolute Charge Mobility of Molecular Semiconductors: the Case of Rubrene. Adv. Mater. 2007, 19, 2000-2004.

(283) Cheng, Y.-C.; Silbey, R. J. A Unified Theory for Charge-Carrier Transport in Organic Crystals. J. Chem. Phys. 2008, 128, 114713.

(284) Ortmann, F.; Bechstedt, F.; Hannewald, K. Theory of Charge Transport in Organic Crystals: Beyond Holstein's Small-Polaron Model. Phys. Rev. B 2009, 79, 235206.

(285) Ortmann, F.; Bechstedt, F.; Hannewald, K. Charge Transport in Organic Crystals: Theory and Modelling. Phys. Status Solidi (b) 2011, 248, 511-525.
(286) Troisi, A. Charge Transport in High Mobility Molecular Semiconductors: Classical Models and New Theories. Chem. Soc. Rev. 2011, 40, 2347-2358.

(287) Girlando, A.; Masino, M.; Visentini, G.; Della Valle, R. G.; Brillante, A.; Venuti, E. Lattice Dynamics and Electron-Phonon Coupling in the $\beta$ (BEDT-TTF)2I3 Organic Superconductor. Phys. Rev. B 2000, 62, 14476.

(288) Girlando, A.; Grisanti, L.; Masino, M.; Bilotti, I.; Brillante, A.; Della Valle, R. G.; Venuti, E. Peierls and Holstein Carrier-Phonon Coupling in Crystalline Rubrene. Phys. Rev. B 2010, 82, 035208.

(289) Li, X.; Tully, J. C.; Schlegel, H. B.; Frisch, M. J. Ab Initio Ehrenfest Dynamics. J. Chem. Phys. 2005, 123, 084106.

(290) Tully, J. C. Molecular Dynamics with Electronic Transitions. J. Chem. Phys. 1990, 93, 1061-1071.

(291) Tully, J. C. In Classical and Quantum Dynamics in Condensed Phase Simulations; Berne, B. J., Ciccotti, G., Coker, D. F., Eds.; World Scientific, 1998; pp 34-71.

(292) Tully, J. C. Perspective: Nonadiabatic Dynamics Theory. J. Chem. Phys. 2012, 137, 22 A301.

(293) Plasser, F.; Lischka, H. Semiclassical Dynamics Simulations of Charge Transport in Stacked $\pi$-systems. J. Chem. Phys. 2011, 134, 034309.

(294) Mitrić, R.; Bonačić-Kouteckỳ, V.; Pittner, J.; Lischka, H. Ab Initio Nonadiabatic Dynamics Study of Ultrafast Radiationless Decay over Conical Intersections Illustrated on the Na3F Cluster. J. Chem. Phys. 2006, 125, 024303.

(295) Blancafort, L.; Hunt, P.; Robb, M. A. Intramolecular Electron Transfer in Bis(methylene)adamantyl Radical 
Cation: A Case study of Diabatic Trapping. J. Am. Chem. Soc. 2005, 127, 3391-3399.

(296) Plasser, F.; Granucci, G.; Pittner, J.; Barbatti, M.; Persico, M.; Lischka, H. Surface Hopping Dynamics Using a Locally Diabatic Formalism: Charge Transfer in the Ethylene Dimer Cation and Excited State Dynamics in the 2-pyridone Dimer. J. Chem. Phys. 2012, 13\%, $22 \mathrm{~A} 514$.

(297) Craig, C. F.; Duncan, W. R.; Prezhdo, O. V. Trajectory Surface Hopping in the Time-Dependent KohnSham Approach for Electron-Nuclear Dynamics. Phys. Rev. Lett. 2005, 95, 163001.

(298) Tapavicza, E.; Tavernelli, I.; Rothlisberger, U. Trajectory Surface Hopping within Linear Response Time-Dependent Density-Functional Theory. Phys. Rev. Lett. 2007, 98, 023001.

(299) Curchod, B.; Penfold, T.; Rothlisberger, U.; Tavernelli, I. Nonadiabatic Ab Initio Molecular Dynamics Using Linear-Response Time-Dependent Density Functional Theory. Cent. Eur. J. Phys. 2013, 11, 1059-1065.

(300) Doltsinis, N. L.; Marx, D. Nonadiabatic Car-Parrinello Molecular Dynamics. Phys. Rev. Lett. 2002, 88, 166402.

(301) Doltsinis, N. L.; Marx, D. First Principles Molecular Dynamics Involving Excited States and Nonadiabatic Transitions. J. Theor. Comp. Chem. 2002, 1, 319-349.

(302) Fabiano, E.; Keal, T.; Thiel, W. Implementation of Surface Hopping Molecular Dynamics Using Semiempirical Methods. Chem. Phys. 2008, 349, 334-347.

(303) Lan, Z.; Fabiano, E.; Thiel, W. Photoinduced Nonadiabatic Dynamics of Pyrimidine Nucleobases: On-the-Fly
Surface-Hopping Study with Semiempirical Methods. J. Phys. Chem. B 2009, 113, 3548-3555.

(304) Nelson, T.; Fernandez-Alberti, S.; Chernyak, V.; Roitberg, A. E.; Tretiak, S. Nonadiabatic Excited-State Molecular Dynamics Modeling of Photoinduced Dynamics in Conjugated Molecules. J. Phys. Chem. B 2011, 115, 5402-5414.

(305) Nelson, T.; Fernandez-Alberti, S.; Roitberg, A. E.; Tretiak, S. Nonadiabatic Excited-State Molecular Dynamics: Modeling Photophysics in Organic Conjugated Materials. Acc. Chem. Res. 2014, 47, 1155-1164.

(306) Sterpone, F.; Rossky, P. J. Molecular Modeling and Simulation of Conjugated Polymer Oligomers: Ground and Excited State Chain Dynamics of PPV in the Gas Phase. J. Phys. Chem. B 2008, 112, 4983-4993.

(307) Elstner, M.; Porezag, D.; Jungnickel, G.; Elsner, J.; Haugk, M.; Frauenheim, T.; Suhai, S.; Seifert, G. Self-ConsistentCharge Density-Functional TightBinding Method for Simulations of Complex Materials Properties. Phys. Rev. B 1998, 58, 7260-7268.

(308) Kubař, T.; Elstner, M. Efficient Algorithms for the Simulation of NonAdiabatic Electron Transfer in Complex Molecular Systems: Application to DNA. Phys. Chem. Chem. Phys. 2013, 15, 5794-5813.

(309) Kubař, T.; Elstner, M. A Hybrid Approach to Simulation of Electron Transfer in Complex Molecular Systems. J. R. Soc. Interface 2013, 10, 20130415.

(310) Mitric, R.; Werner, U.; Wohlgemuth, M.; Seifert, G.; Bonačić-Koutecký, V. Nonadiabatic Dynamics within TimeDependent Density Functional Tight Binding Method. J. Phys. Chem. A 2009, 113, 12700-12705. 
(311) Wang, L.; Beljonne, D. Charge Transport in Organic Semiconductors: Assessment of the Mean Field Theory in the Hopping Regime. J. Chem. Phys. 2013, 139, 064316.

(312) Press, W. H.; Teukolsky, S. A.; Vetterling, W. T.; Flannery, B. P. Numerical Recipes, 3rd ed.; Cambridge University Press: Cambridge, 2007.

(313) Schleife, A.; Draeger, E. W.; Kanai, Y.; Correa, A. A. Plane-Wave Pseudopotential Implementation of Explicit Integrators for Time-Dependent Kohn-Sham Equations in Large-Scale Simulations. J. Chem. Phys. 2012, 13\%, 22A546.

(314) Castro, A.; Marques, M. A. TimeDependent Density Functional Theory; Springer, 2006; pp 197-210.

(315) Wang, L.; Beljonne, D.; Chen, L.; Shi, Q. Mixed Quantum-Classical Simulations of Charge Transport in Organic Materials: Numerical Benchmark of the SuSchrieffer-Heeger Model. J. Chem. Phys. 2011, 134, 244116.

(316) Troisi, A.; Orlandi, G. Charge-Transport Regime of Crystalline Organic Semiconductors: Diffusion Limited by Thermal Off-Diagonal Electronic Disorder. Phys. Rev. Lett. 2006, 96, 086601.

(317) Hultell, M.; Stafström, S. Polaron Dynamics in Highly Ordered Molecular Crystals. Chem. Phys. Lett. 2006, 428, 446-450.

(318) Castro, A.; Gross, E. Optimal Control Theory for Quantum-Classical Systems: Ehrenfest Molecular Dynamics Based on Time-Dependent Density-Functional Theory. J. Phys. A 2013, 47, 025204.

(319) Akimov, A. V.; Long, R.; Prezhdo, O. V. Coherence Penalty Functional: A Simple Method for Adding Decoherence in Ehrenfest Dynamics. J. Chem. Phys. 2014, 140, 194107.
(320) Granucci, G.; Persico, M. Critical Appraisal of the Fewest Switches Algorithm for Surface Hopping. J. Chem. Phys. 2007, 126, 134114.

(321) Wang, L.; Akimov, A. V.; Prezhdo, O. V. Recent Progress in Surface Hopping: 2011-2015. J. Phys. Chem. Lett. 2016, 7, 2100-2112.

(322) Bittner, E. R.; Rossky, P. J. Quantum Decoherence in Mixed QuantumClassical Systems: Nonadiabatic Processes. J. Chem. Phys. 1995, 103, 81308143 .

(323) Fang, J.-Y.; Hammes-Schiffer, S. Improvement of the Internal Consistency in Trajectory Surface Hopping. J. Phys. Chem. A 1999, 103, 9399-9407.

(324) Hack, M. D.; Wensmann, A. M.; Truhlar, D. G.; Ben-Nun, M.; Martınez, T. J. Comparison of Full Multiple Spawning, Trajectory Surface Hopping, and Converged Quantum Mechanics for Electronically Nonadiabatic Dynamics. J. Chem. Phys. 2001, 115, 1172-1186.

(325) Subotnik, J. E.; Shenvi, N. A New Approach to Decoherence and Momentum Rescaling in the Surface Hopping Algorithm. J. Chem. Phys. 2011, 134, 024105 .

(326) Subotnik, J. E.; Shenvi, N. Decoherence and Surface Hopping: When Can Averaging over Initial Conditions Help Capture the Effects of Wave Packet Separation? J. Chem. Phys. 2011, 134, 244114.

(327) Shushkov, P.; Li, R.; Tully, J. C. Ring Polymer Molecular Dynamics with Surface Hopping. J. Chem. Phys. 2012, 137, $22 \mathrm{~A} 549$.

(328) Habershon, S.; Manolopoulos, D. E.; Markland, T. E.; Miller III, T. F. RingPolymer Molecular Dynamics: Quantum Effects in Chemical Dynamics from Classical Trajectories in an Extended Phase 
Space. Annu. Rev. Phys. Chem. 2013, 64, 387-413.

(329) Lu, J.; Zhou, Z. Path Integral Molecular Dynamics with Surface Hopping for Thermal Equilibrium Sampling of Nonadiabatic Systems. J. Chem. Phys. 2017, 146,154110 .

(330) Schwartz, B. J.; Bittner, E. R.; Prezhdo, O. V.; Rossky, P. J. Quantum Decoherence and the Isotope Effect in Condensed Phase Nonadiabatic Molecular Dynamics Simulations. J. Chem. Phys. 1996, 104, 5942-5955.

(331) Zhu, C.; Nangia, S.; Jasper, A. W.; Truhlar, D. G. Coherent Switching with Decay of Mixing: An Improved Treatment of Electronic Coherence for Non-BornOppenheimer Trajectories. J. Chem. Phys. 2004, 121, 7658-7670.

(332) Landry, B. R.; Subotnik, J. E. Communication: Standard Surface Hopping Predicts Incorrect Scaling for Marcus' Golden-Rule Rate: The Decoherence Problem Cannot be Ignored. J. Chem. Phys. 2011, 135, 191101.

(333) Chen, H.-T.; Reichman, D. R. On the Accuracy of Surface Hopping Dynamics in Condensed Phase Non-Adiabatic Problems. J. Chem. Phys. 2016, 144, 094104.

(334) Landry, B. R.; Subotnik, J. E. How to Recover Marcus Theory with Fewest Switches Surface Hopping: Add Just a Touch of Decoherence. J. Chem. Phys. 2012, 137, 22A513.

(335) Falk, M. J.; Landry, B. R.; Subotnik, J. E. Can Surface Hopping sans Decoherence Recover Marcus Theory? Understanding the Role of Friction in a Surface Hopping View of Electron Transfer. J. Phys. Chem. B 2014, 118, 8108-8117.

(336) Worth, G. A.; Cederbaum, L. S. Beyond Born-Oppenheimer: Molecular Dynamics through a Conical Intersection. Annu. Rev. Phys. Chem. 2004, 55, 127-158.
(337) Fernandez-Alberti, S.; Roitberg, A. E.; Nelson, T.; Tretiak, S. Identification of Unavoided Crossings in Nonadiabatic Photoexcited Dynamics Involving Multiple Electronic States in Polyatomic Conjugated Molecules. J. Chem. Phys. 2012, $137,014512$.

(338) Wang, L.; Prezhdo, O. V. A Simple Solution to the Trivial Crossing Problem in Surface Hopping. J. Phys. Chem. Lett. 2014, 5, 713-719.

(339) Hurd, P.; Cusati, T.; Persico, M. Trajectory Integration with Potential Energy Discontinuities. J. Chem. Phys. 2010, 229, 2109-2116.

(340) Schmidt, J.; Parandekar, P. V.; Tully, J. C. Mixed Quantum-Classical Equilibrium: Surface Hopping. J. Chem. Phys. 2008, 129, 044104.

(341) Sherman, M.; Corcelli, S. Thermal Equilibrium Properties of Surface Hopping with an Implicit Langevin Bath. $J$. Chem. Phys. 2015, 142, 024110.

(342) Shenvi, N.; Cheng, H.; Tully, J. C. Nonadiabatic Dynamics near Metal Surfaces: Decoupling Quantum Equations of Motion in the Wide-Band Limit. Phys. Rev. A 2006, 74, 062902.

(343) Provorse, M. R.; Isborn, C. M. Electron Dynamics with Real-Time TimeDependent Density Functional Theory. Int. J. Quantum Chem. 2016, 739-749.

(344) Runge, E.; Gross, E. K. U. DensityFunctional Theory for Time-Dependent Systems. Phys. Rev. Lett. 1984, 52, 9971000 .

(345) Hohenberg, P.; Kohn, W. Inhomogeneous Electron Gas. Phys. Rev. 1964, 136, B864-B871.

(346) van Leeuwen, R. Mapping from Densities to Potentials in Time-Dependent Density-Functional Theory. Phys. Rev. Lett. 1999, 82, 3863. 
(347) Kohn, W.; Sham, L. J. Self-Consistent Equations Including Exchange and Correlation Effects. Phys. Rev. 1965, 140, A1133-A1138.

(348) Maitra, N. T.; Burke, K.; Woodward, C. Memory in Time-Dependent Density Functional Theory. Phys. Rev. Lett. 2002, 89, 023002.

(349) Marques, M. A.; Maitra, N. T.; Nogueira, F. M.; Gross, E. K.; Rubio, A. Fundamentals of Time-dependent Density Functional Theory; Springer Science \& Business Media, 2012; Vol. 837.

(350) Thiele, M.; Gross, E.; Kümmel, S. Adiabatic Approximation in Nonperturbative Time-Dependent Density-Functional Theory. Phys. Rev. Lett. 2008, 100, 153004 .

(351) Elliott, P.; Fuks, J. I.; Rubio, A.; Maitra, N. T. Universal Dynamical Steps in the Exact Time-Dependent ExchangeCorrelation Potential. Phys. Rev. Lett. 2012, 109, 266404.

(352) Fuks, J. I.; Elliott, P.; Rubio, A.; Maitra, N. T. Dynamics of ChargeTransfer Processes with Time-Dependent Density Functional Theory. J. Phys. Chem. Lett. 2013, 4, 735-739.

(353) Fuks, J.; Maitra, N. Charge Transfer in Time-Dependent Density-Functional Theory: Insights from the Asymmetric Hubbard Dimer. Phys. Rev. A 2014, 89, 062502 .

(354) Castro, A.; Marques, M. A.; Alonso, J. A.; Bertsch, G. F.; Rubio, A. Excited States Dynamics in Time-Dependent Density Functional Theory. Eur. Phys. J. D 2004, 28, 211-218.

(355) Alonso, J. L.; Andrade, X.; Echenique, P.; Falceto, F.; PradaGracia, D.; Rubio, A. Efficient Formalism for Large-Scale Ab Initio Molecular Dynamics Based on Time-Dependent
Density Functional Theory. Phys. Rev. Lett. 2008, 101, 096403.

(356) Kreibich, T.; Gross, E. Multicomponent Density-Functional Theory for Electrons and Nuclei. Phys. Rev. Lett. 2001, 86, 2984 .

(357) Butriy, O.; Ebadi, H.; de Boeij, P.; van Leeuwen, R.; Gross, E. Multicomponent Density-Functional Theory for Time-Dependent Systems. Phys. Rev. A 2007, 76, 052514.

(358) Makri, N.; Miller, W. H. TimeDependent Self-Consistent Field (TD$\mathrm{SCF}$ ) Approximation for a Reaction Coordinate Coupled to a Harmonic Bath: Single and Multiple Configuration Treatments. J. Chem. Phys. 1987, 87, $5781-5787$.

(359) Meyer, H.-D.; Manthe, U.; Cederbaum, L. S. The Multi-Configurational Time-Dependent Hartree Approach. Chem. Phys. Lett. 1990, 165, 73-78.

(360) Manthe, U.; Meyer, H.-D.; Cederbaum, L. S. Wave-Packet Dynamics within the Multiconfiguration Hartree Framework: General Aspects and Application to NOCl. J. Chem. Phys. 1992, 97, 3199-3213.

(361) Beck, M. H.; Jäckle, A.; Worth, G.; Meyer, H.-D. The Multiconfiguration Time-Dependent Hartree (MCTDH) Method: A Highly Efficient Algorithm for Propagating Wavepackets. Phys. reports 2000, 324, 1-105.

(362) Worth, G.; Meyer, H.-D.; Köppel, H.; Cederbaum, L.; Burghardt, I. Using the MCTDH Wavepacket Propagation Method to Describe Multimode NonAdiabatic Dynamics. Int. Rev. Phys. Chem. 2008, 27, 569-606.

(363) Wang, H.; Thoss, M. Multilayer Formulation of the Multiconfiguration TimeDependent Hartree Theory. J. Chem. Phys. 2003, 119, 1289-1299. 
(364) Manthe, U. A Multilayer Multiconfigurational Time-Dependent Hartree Approach for Quantum Dynamics on General Potential Energy Surfaces. J. Chem. Phys. 2008, 128, 164116.

(365) Vendrell, O.; Meyer, H.-D. Multilayer Multiconfiguration Time-Dependent Hartree Method: Implementation and Applications to a Henon-Heiles Hamiltonian and to Pyrazine. J. Chem. Phys. 2011, 134, 044135.

(366) Tamura, H.; Martinazzo, R.; Ruckenbauer, M.; Burghardt, I. Quantum Dynamics of Ultrafast Charge Transfer at an Oligothiophene-Fullerene Heterojunction. J. Chem. Phys. 2012, 137, 22A540.

(367) Binder, R.; Wahl, J.; Römer, S.; Burghardt, I. Coherent Exciton Transport Driven by Torsional Dynamics: A Quantum Dynamical Study of Phenylene-Vinylene Type Conjugated Systems. Farad. Discuss. 2013, 163, 205-222.

(368) Tamura, H.; Burghardt, I. Potential Barrier and Excess Energy for Electron-Hole Separation from the Charge-Transfer Exciton at Donor-Acceptor Heterojunctions of Organic Solar Cells. J. Phys. Chem. C 2013, 117, 15020-15025.

(369) Zheng, J.; Xie, Y.; Jiang, S.; Lan, Z. Ultrafast Nonadiabatic Dynamics of Singlet Fission: Quantum Dynamics with the Multilayer Multiconfigurational Time-Dependent Hartree (ML-MCTDH) Method. J. Phys. Chem. C 2016, 120, 1375-1389.

(370) Tan, D.; Chen, Z. On a General Formula of Fourth Order Runge-Kutta Method. J. Math. Sci. Math. Educ. 2012, 7, 110.

(371) Mori-Sánchez, P.; Cohen, A. J.; Yang, W. Localization and Delocalization Errors in Density Functional Theory and Implications for Band-Gap Prediction. Phys. Rev. Lett. 2008, 100, 146401.
(372) Kubo, R. Statistical-Mechanical Theory of Irreversible Processes. I. General Theory and Simple Applications to Magnetic and Conduction Problems. J. Phys. Soc. Jpn. 1957, 12, 570-586.

(373) Gunnarsson, O.; Han, J. The Mean Free Path for Electron Conduction in Metallic Fullerenes. Nature 2000, 405, 1027 1030 .

(374) Wang, Y.; MacKernan, D.; Cubero, D.; Coker, D. F.; Quirke, N. Single Electron States in Polyethylene. J. Chem. Phys. 2014, 140, 154902.

(375) Greenwood, D. A. The Boltzmann Equation in the Theory of Electrical Conduction in Metals. Proc. Phys. Soc 1958, 71, $585-596$.

(376) Dura, J.; Triozon, F.; Barraud, S.; Munteanu, D.; Martinie, S.; Autran, J. L. Kubo-Greenwood Approach for the Calculation of Mobility in Gate-All-Around Nanowire MetalOxide-Semiconductor Field-Effect Transistors including Screened Remote Coulomb Scattering - Comparison with Experiment. J. Appl. Phys. 2012, 111, 103710.

(377) Ren, J.; Vukmirović, N.; Wang, L.W. Nonadiabatic Molecular Dynamics Simulation for Carrier Transport in a Pentathiophene Butyric Acid Monolayer. Phys. Rev. B 2013, 87, 205117.

(378) Wang, L.; Akimov, A. V.; Chen, L.; Prezhdo, O. V. Quantized Hamiltonian Dynamics Captures the LowTemperature Regime of Charge Transport in Molecular Crystals. J. Chem. Phys. 2013, 139, 174109.

(379) Zhang, W.; Zhong, X.; Zhao, Y. Electron Mobilities of N-Type Organic Semiconductors from Time-Dependent Wavepacket Diffusion Method: Pentacenequinone Derivatives. J. Phys. Chem. A 2012, 116, 11075-11082. 
(380) Wilcox, D. E.; Lee, M. H.; Sykes, M. E.; Niedringhaus, A.; Geva, E.; Dunietz, B. D.; Shtein, M.; Ogilvie, J. P. Ultrafast Charge-Transfer Dynamics at the Boron Subphthalocyanine Chloride/C60 Heterojunction: Comparison between Experiment and Theory. J. Phys. Chem. Lett. 2015, 6, 569-575.

(381) Johansson, A. A.; Stafström, S. Nonadiabatic Simulations of Polaron Dynamics. Phys. Rev. B 2004, 69, 235205.

(382) Johansson, Å.; Stafström, S. Polaron Dynamics in a System of Coupled Conjugated Polymer Chains. Phys. Rev. Lett. 2001, 86, 3602.

(383) An, Z.; Wu, C.; Sun, X. Dynamics of Photogenerated Polarons in Conjugated Polymers. Phys. Rev. Lett. 2004, 93, 216407.

(384) Bauer, T.; Jäger, C. M.; Jordan, M. J. T.; Clark, T. A Multi-Agent Quantum Monte Carlo Model for Charge Transport: Application to Organic Field-Effect Transistors. J. Chem. Phys. 2015, 143, 044114. 